\title{
Plant pathogens convergently
} evolved to counteract redundant nodes of an NLR immune receptor network

\section{Journal Article}

\section{Author(s):}

Derevnina, Lida; Contreras, Mauricio P.; Adachi, Hiroaki; Upson, Jessica; Vergara Cruces, Angel; Xie, Rongrong; Sklenar, Jan; Menke, Frank L.H.; Mugford, Sam T.; MacLean, Dan; Ma, Wenbo; Hogenhout, Saskia; Goverse, Aska; Maqbool, Abbas; Wu, ChihHang; Kamoun, Sophien

Publication date:

2021-08

Permanent link:

https://doi.org/10.3929/ethz-b-000502775

\section{Rights / license:}

Creative Commons Attribution 4.0 International

\section{Originally published in:}

PLoS Biology 19(8), https://doi.org/10.1371/journal.pbio.3001136 


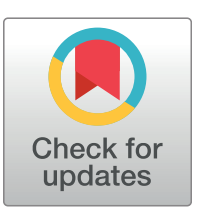

\section{OPEn ACCESS}

Citation: Derevnina L, Contreras MP, Adachi H, Upson J, Vergara Cruces A, Xie R, et al. (2021) Plant pathogens convergently evolved to counteract redundant nodes of an NLR immune receptor network. PLoS Biol 19(8): e3001136. https://doi.org/10.1371/journal.pbio.3001136

Academic Editor: Xinnian Dong, Duke University, UNITED STATES

Received: February 8, 2021

Accepted: July 27, 2021

Published: August 23, 2021

Peer Review History: PLOS recognizes the benefits of transparency in the peer review process; therefore, we enable the publication of all of the content of peer review and author responses alongside final, published articles. The editorial history of this article is available here: https://doi.org/10.1371/journal.pbio.3001136

Copyright: @ 2021 Derevnina et al. This is an open access article distributed under the terms of the Creative Commons Attribution License, which permits unrestricted use, distribution, and reproduction in any medium, provided the original author and source are credited.

Data Availability Statement: All relevant data are within the paper and its Supporting Information files. The MS proteomics data have been deposited to the ProteomeXchange Consortium via the

\section{Plant pathogens convergently evolved to counteract redundant nodes of an NLR immune receptor network}

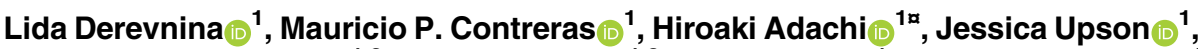 \\ Angel Vergara Cruces ${ }^{1,2}$, Rongrong Xie ${ }^{1,3}$, Jan Skłenar ${ }^{1}{ }^{1}$, Frank L. H. Menke ${ }^{1}{ }^{1}$, Sam \\ T. Mugford $\mathbb{D}^{4}$, Dan MacLean ${ }_{(\mathbb{D}}{ }^{1}$, Wenbo Ma ${ }_{(\mathbb{D}}^{1,5}$, Saskia A. Hogenhout $\mathbb{D}^{4}$, \\ Aska Goverse $\mathbb{D}^{6}$, Abbas Maqbool $\mathbb{D}^{1}$, Chih-Hang $W u_{(\mathbb{D}}{ }^{1,7 *}$, Sophien Kamoun $\mathbb{D}^{1 *}$ \\ 1 The Sainsbury Laboratory, University of East Anglia, Norwich, United Kingdom, 2 Department of Biology, \\ Swiss Federal Institute of Technology (ETH), Zürich, Switzerland, 3 Joint Center for Single Cell Biology, \\ School of Agriculture and Biology, Shanghai, Jiao Tong University, Shanghai, China, 4 Department of Crop \\ Genetics, John Innes Centre, Norwich, United Kingdom, 5 Department of Plant Pathology and Microbiology, \\ University of California, Riverside, California, United States of America, 6 Laboratory of Nematology, \\ Wageningen University and Research, Wageningen, the Netherlands, 7 Institute of Plant and Microbial \\ Biology, Academia Sinica, Taipei, Taiwan \\ a Current address: Graduate School of Biological Sciences, Nara Institute of Science and Technology, \\ Ikoma, Japan \\ *wuchh@gate.sinica.edu.tw (C-HW); Sophien.Kamoun@tsl.ac.uk (SK)
}

\section{Abstract}

In plants, nucleotide-binding domain and leucine-rich repeat (NLR)-containing proteins can form receptor networks to confer hypersensitive cell death and innate immunity. One class of NLRs, known as NLR required for cell death (NRCs), are central nodes in a complex network that protects against multiple pathogens and comprises up to half of the NLRome of solanaceous plants. Given the prevalence of this NLR network, we hypothesised that pathogens convergently evolved to secrete effectors that target NRC activities. To test this, we screened a library of 165 bacterial, oomycete, nematode, and aphid effectors for their capacity to suppress the cell death response triggered by the NRC-dependent disease resistance proteins Prf and Rpi-blb2. Among 5 of the identified suppressors, 1 cyst nematode protein and 1 oomycete protein suppress the activity of autoimmune mutants of NRC2 and NRC3, but not NRC4, indicating that they specifically counteract a subset of NRC proteins independently of their sensor NLR partners. Whereas the cyst nematode effector SPRYSEC15 binds the nucleotide-binding domain of NRC2 and NRC3, the oomycete effector AVRcap1b suppresses the response of these NRCs via the membrane trafficking-associated protein NbTOL9a (Target of Myb 1-like protein 9a). We conclude that plant pathogens have evolved to counteract central nodes of the NRC immune receptor network through different mechanisms. Coevolution with pathogen effectors may have driven NRC diversification into functionally redundant nodes in a massively expanded NLR network. 
PRIDE partner repository with the dataset identifier PXD023178 and 10.6019/PXD023178.

\section{Funding: This work was funded by the Gatsby Charitable Foundation (Core grant to TSL) and Biotechnology and Biological Sciences Research Council (BBSRC, UK) BB/P012574 and BB/ V002937/1. S.K. also receives funding from the European Research Council (ERC NGRB and BLASTOFF projects). L.D. was funded by a Marie Sklodowska-Curie actions fellowship (BoostR), $\mathrm{H}$. A. was funded by the Japan Society for the Promotion of Sciences Postdoctoral fellowship, A. V.C. was funded by a British Society for Plant Pathology summer bursary, J.U. was funded by the Gatsby Charitable Foundation PhD studentship. The funders had no role in study design, data collection and analysis, decision to publish, or preparation of the manuscript.}

Competing interests: I have read the journal's policy and the authors of this manuscript have the following competing interests: S.K. receives funding from industry on NLR biology.

Abbreviations: AVR, avirulence; CaMV, cauliflower mosaic virus; $\mathrm{CC}$, coiled-coil; colP, coimmunoprecipitation; $\mathrm{CP}$, coat protein; ESCRT, endosomal sorting complex required for transport; ETI, effector-triggered immunity; EV, empty vector; $H R$, hypersensitive response; HRP, horse radish peroxidase; LC-MS/MS, liquid chromatographytandem mass spectrometry; LRR, leucine-rich repeat; MAPKK, mitogen-activated protein kinase kinase; MHD, methionine-histidine-aspartate; NBARC, nucleotide-binding domain shared with APAF-1, various R-proteins, and CED-4; NLR, nucleotide-binding domain and leucine-rich repeat; NRC, NLR required for cell death; NTI, NLRtriggered immunity; PAMP, pathogen-associated molecular pattern; PRR, pattern recognition receptor; PTI, PRR-triggered immunity; PVX, Potato virus $X$; R0Q1, Recognition of XopQ 1; RPP1, Recognition of Peronospora parasitica 1; TIR, Toll/interleukin-1 receptor; TOL, Target of Myb 1-like protein; TRV, Tobacco rattle virus; VIGS, virus-induced gene silencing; $\mathrm{Y} 2 \mathrm{H}$, yeast twohybrid; ZAR1, HopZ-activated resistance 1.

\section{Introduction}

Our view of the pathogenicity mechanisms of plant pathogens and pests has significantly broadened over the years. Parasites as diverse as bacteria, oomycetes, nematodes, and aphids turned out to be much more sophisticated manipulators of their host plants than initially anticipated. Indeed, it is now well established that these parasites secrete an arsenal of proteins, termed effectors, which modulate plant responses, such as innate immunity, to enable host infection and colonisation. As a consequence, deciphering the biochemical activities of effectors to understand how parasites successfully colonise and reproduce has become a major conceptual paradigm in the field of molecular plant pathology [1,2]. In fact, effectors have emerged as molecular probes that can be utilised to unravel novel components and processes of the host immune system [2].

Most effectors studied to date suppress immune pathways induced by pathogen-associated molecular patterns (PAMPs). This so-called PAMP or pattern recognition receptor (PRR)triggered immunity (PTI) response is mediated by cell surface PRRs [3,4] (S1A Fig). A subset of effectors, however, have avirulence (AVR) activity and inadvertently activate intracellular immune receptors of the nucleotide-binding, leucine-rich repeat (NLR) class of proteins, a response known as effector- or NLR-triggered immunity (ETI/NTI) [5-7]. In plants, recognition of AVR effectors by NLRs can occur either directly or indirectly following various mechanistic models $[8,9]$. NTI is usually accompanied by a localised form of programmed cell death known as the hypersensitive response (HR) that hinders disease progression [6,7,10,11]. NLRmediated immunity activated by AVR effectors can also be suppressed by other effectors [12]. However, in sharp contrast to the widely studied PTI-suppressing effectors, the mechanisms by which effectors suppress NLR responses remain poorly understood $[3,13,14]$. Therefore, understanding how effectors suppress NLR functions should provide important insights into the black box of how these immune receptors activate cell death and innate immunity, one of the major unsolved questions in the field of plant pathology [15].

NLRs are multidomain proteins with a nucleotide-binding domain shared with APAF-1, various R-proteins, and CED-4 (NB-ARC) and at least 1 additional domain [16]. The C-terminus of plant NLRs is generally a leucine-rich repeat (LRR) domain, but they can be sorted into phylogenetic subgroups with distinct $\mathrm{N}$-terminal domains [16-18]. In angiosperms, NLRs form several major phylogenetic subgroups, including TIR-NLRs with an N-terminal Toll/ interleukin-1 receptor (TIR) domain, CC-NLRs, with the Rx-type coiled-coil (CC) domain, $\mathrm{CC}_{\mathrm{R}}$-NLRs with the RPW8-type $\mathrm{CC}\left(\mathrm{CC}_{\mathrm{R}}\right)$ domain, and the $\mathrm{CC}_{\mathrm{G} 10}-\mathrm{NLR}$ subclade with a distinct type of $\mathrm{CC}\left(\mathrm{CC}_{\mathrm{A} \text { or }} \mathrm{CC}_{\mathrm{G} 10}\right)$ domain. Of these, $\mathrm{CC}$-NLRs are the most common type, forming the largest group of NLRs in angiosperms [16,18,19]. The TIR- and CC-type N-terminal domains are involved in downstream immune signalling, oligomerization, and cell death execution [20]. The central NB-ARC domain exhibits ATP binding and hydrolysis activities and functions as a molecular switch that determines the NLR inactive/active status [21]. Finally, the LRR domain can mediate effector perception and often engages in intramolecular interactions $[8,21]$.

Plant genomes may encode anywhere between 50 and approximately 1,000 NLRs [17,22]. Some of these NLRs are functional singletons operating as single biochemical units, while others function in pairs or in more complex receptor networks [23]. Paired and networked NLRs consist of functionally specialised sensor NLRs that detect pathogen effectors and helper NLRs that translate this effector recognition into hypersensitive cell death and resistance. In the Solanaceae, a major phylogenetic clade of NLRs forms a complex immunoreceptor network in which multiple helper CC-NLRs, known as NLR required for cell death (NRCs), are necessary for a large number of sensor NLRs [24]. These sensor NLRs, encoded by R gene loci, confer 
resistance against diverse pathogens, such as viruses, bacteria, oomycetes, nematodes, and insects [24] (S1B Fig). Together, NRCs and their NLR partners form the NRC superclade, a well-supported phylogenetic cluster divided into an NRC helper clade and 2 larger clades that include all known NRC-dependent sensor NLRs [24,25]. The NRC superclade emerged over 100 million years ago (Mya) from an NLR pair that expanded to make up a significant fraction of the NLRome of asterid plant species [24]. The current model is that NRCs form redundant central nodes in this massively diversified bow tie network, with different NRCs exhibiting some specificity towards their sensor NLR partners. For example, whereas the bacterial resistance protein Prf requires NRC2 and NRC3 in a genetically redundant manner, the potato blight resistance protein Rpi-blb2 is dependent only on NRC4 [24,26] (S1B Fig).

Until very recently, the molecular mechanisms that underpin plant NLR activation and the subsequent execution of NTI pathways and hypersensitive cell death have remained unknown. The recent elucidation of the CC-NLR ZAR1 (HopZ-activated resistance 1) and the TIR-NLRs ROQ1 (Recognition of XopQ 1) and RPP1 (Recognition of Peronospora parasitica 1) structures have resulted in a new conceptual framework [27-30]. Activated NLRs oligomerize into a wheel-like multimeric complex known as the resistosome. In the case of ZAR1, activation induces conformational changes in the NB-ARC domain resulting in ADP release followed by dATP/ATP binding and oligomerization of ZAR1 and its partner host proteins into the pentameric resistosome structure [28,31]. ZAR1 resistosomes expose a funnel-shaped structure formed by the $\mathrm{N}$-terminal $\alpha 1$ helices, which was proposed to perturb plasma membrane integrity to trigger hypersensitive cell death $[28,29]$. This $\alpha 1$ helix is defined by a molecular signature, the MADA motif, which is present in approximately $20 \%$ of angiosperm CC-NLRs, including NRCs [25]. This implies that the biochemical "death switch" mechanism of the ZAR1 resistosome probably applies to NRCs and other MADA motif containing NLRs across diverse plant taxa.

The NRC superclade is massively expanded in solanaceous plants, where it comprises up to half of the NLRome in some species [24]. Given this prevalence, we hypothesised that pathogens convergently evolved to secrete effectors that target the NRC network. In this study, we screened collections of effector proteins from 6 major parasites of solanaceous plants, i.e., the bacterium Pseudomonas syringae, the oomycete Phytophthora infestans, the cyst nematodes Globodera rostochiensis and Globodera pallida, and the aphids Myzus persicae and Acrythosiphon pisum, for their capacity to suppress the hypersensitive cell death triggered by the NRCdependent sensor NLRs Prf and Rpi-blb2. These screens revealed 5 effectors as suppressors of components of the NRC network. Among these, the oomycete protein PITG-16705 (PBHR_012, henceforth, AVRcap1b) [32] and the cyst nematode protein SPRYSEC15 (where the term SPRYSEC will be referred to as SS from here on in) stood out for being able to specifically suppress the response triggered by autoimmune mutants of NRC 2 and NRC3, but not NRC4, indicating that they are able to counteract a subset of NRC helper proteins independently of their sensor NLR partners. Further studies revealed that AVRcap1b and SS15 suppress NRC2 and NRC3 through different mechanisms. While AVRcap1b suppression of NRC2 and NRC3 mediated immunity is dependent on the membrane trafficking-associated protein NbTOL9a (Target of Myb 1-like protein 9a), SS15 directly binds the NB-ARC domain of NRC2 and NRC3 to perturb their function. We conclude that evolutionarily divergent plant pathogens have convergently evolved distinct molecular strategies to counteract central nodes of the NRC immune receptor network. Coevolution with pathogen effectors may, therefore, underpin the diversification of NRCs as functionally redundant nodes in a massively expanded NLR network. 


\section{Results}

\section{In planta screens reveal pathogen effectors that suppress NRC-mediated hypersensitive cell death}

To determine the extent to which pathogens have evolved to target the NRC network, we screened candidate effectors from 6 diverse pathogen species for their ability to suppress the hypersensitive cell death triggered by the disease resistance proteins Prf (responds to Pto/ AvrPto; NRC2/3 dependent) and Rpi-blb2 (responds to AVRblb2; NRC4 dependent) [24,26]. The effectors and empty vector (EV, control) were transiently expressed with Pto/AvrPto or Rpi-blb2/AVRblb2 in leaves of the model experimental plant Nicotiana benthamiana and assessed for the absence of cell death, which is indicative of a suppression phenotype (Fig 1A). Our screen included 165 effector candidates from oomycetes ( $P$. infestans, 63), bacteria (P. syringae, 26), cyst nematodes (G. rostochiensis, 23 and G. pallida, 3), and aphids (M. persicae, 47 and A.pisum, 3). The panel of effectors used in the screen was comprised of candidates with previously reported involvement in immune suppression and effectors that were readily available in the lab. Among the 165 effectors tested, 2 effectors (AVRcap1b and SS15) suppressed Prf-mediated cell death, and 3 effectors (PITG-15278, SS10, and SS34) suppressed Rpi-blb2mediated cell death (Figs 1B-1D and S2, S1-S4 Data). AVRcap1b and PITG-15278 are RXLR-WY/LWY domain containing effectors from P. infestans [33,34]. SS10, SS15, and SS34 are SPRY domain containing effectors from G. rostochiensis [35-37] (Fig 2A, S1 Table). Interestingly, HopAB2 (also known as AvrPtoB), which is well known to suppress Prf-mediated cell death [38], was not a robust suppressor in our assays, and only suppressed Prf-mediated cell death in older leaves of N. benthamiana (S3 Fig), leaf age as defined by [39].

\section{AVRcap1b and SS15 effectors suppress the cell death triggered by autoimmune mutants of NRC2 and NRC3 but not NRC4}

To ascertain whether the identified effectors suppress the activity of the sensor NLRs (Prf or Rpiblb2) or the underlying helper NLRs (NRC2, NRC3, and NRC4), we generated constitutively active (autoimmune) NRCs by mutating the methionine-histidine-aspartate (MHD) motif resulting in the NRC autoactive variants $\mathrm{NRC} 2^{\mathrm{H} 480 \mathrm{R}}, \mathrm{NRC}^{\mathrm{D} 480 \mathrm{~V}}$, and $\mathrm{NRC} 4^{\mathrm{D} 478 \mathrm{~V}}$ [40] (Fig 2B). When transiently expressed in N. benthamiana, all 3 NRC mutants caused cell death in the absence of a pathogen AVR effector (Fig 2C). Next, we coexpressed the suppressors AVRcap1b, SS15, PITG-15278, SS10, and SS34, along with the EV control, with $\mathrm{NRC}_{2}{ }^{\mathrm{H} 480 \mathrm{R}}, \mathrm{NRC} 3^{\mathrm{D} 480 \mathrm{~V}}$, and $\mathrm{NRC} 4^{\mathrm{D} 478 \mathrm{~V}}$ in N. benthamiana leaves (S4 Fig, S4 Data). PITG-15278, SS10, and SS34 did not suppress any of the autoactive NRCs, indicating that they target the sensor NLR Rpi-blb2 or the interaction between Rpi-blb2 and NRC4 (S4 Fig). Interestingly, both AVRcap1b and SS15 suppressed the cell death induced by autoimmune NRC2 and NRC3, therefore recapitulating their specific suppression of the sensor NLRs Prf (Figs 2C and 2D and S5, S5 Data). In contrast, AVRcap1b and SS15 did not affect the autoactivity of NRC4 (Figs $2 \mathrm{C}$ and 2D and S5). These results indicate that the suppression activity of AVRcap1b and SS15 is independent of the sensor NLR Prf and is specific to NRC2 and NRC3. In light of these results, we focused on AVRcap1b and SS15 as they are likely acting on the NRC2 and NRC3 helper nodes of the NRC network and thus hold more potential to shed light on the molecular mechanisms underpinning helper NLR activation and downstream signalling.

\section{AVRcap1b and SS15 effectors suppress multiple disease resistance proteins (sensor NLRs) that signal through NRC2 and NRC3}

To further challenge our finding that AVRcap1b and SS15 target NRC2 and NRC3 but not NRC4, we screened the 2 effectors for suppression of 6 disease resistance sensor NLRs (SW5b, 
A

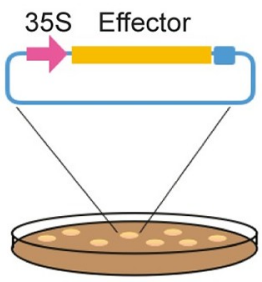

Agrobacterium

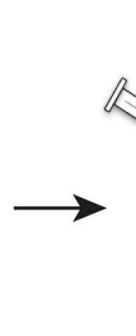

Nicotiana benthamiana

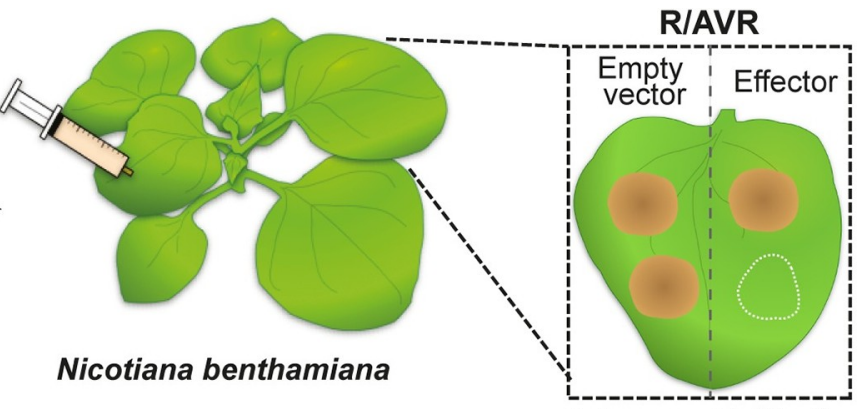

5 days post infiltration
No cell death suppression

Suppression of cell death

B

Pto/AvrPto (Prf, NRC2/3 dependent)

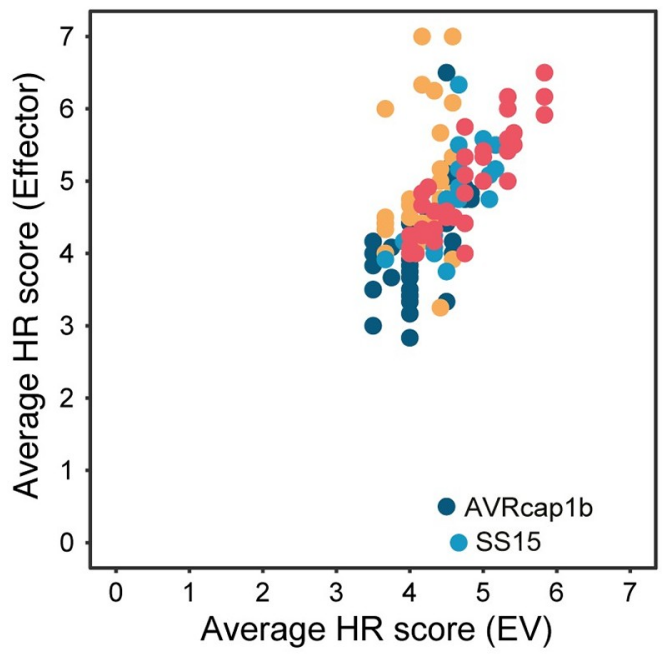

C

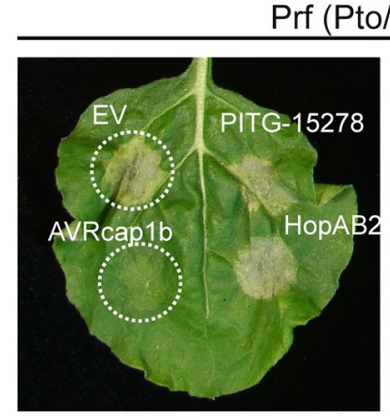

D

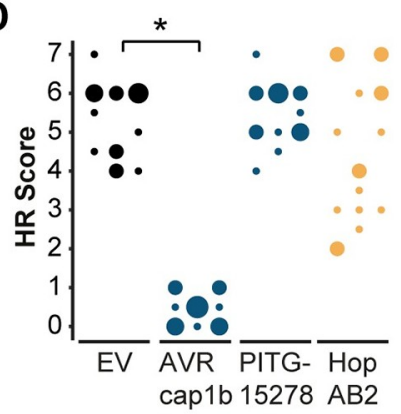

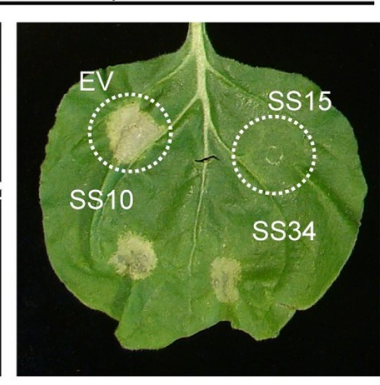

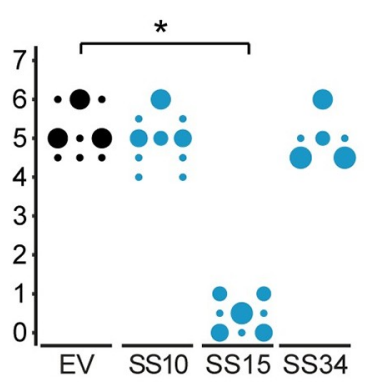

Rpi-blb2/AVRblb2 (NRC4 dependent)

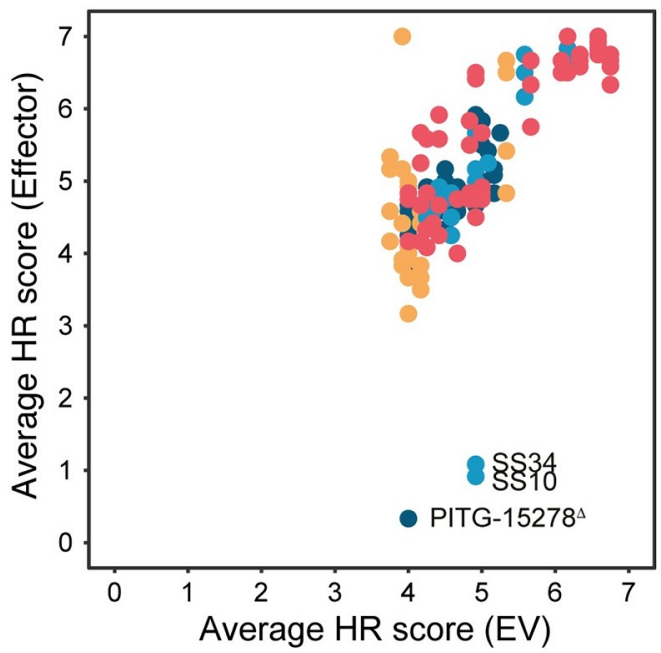

Rpi-blb2/AVRblb2

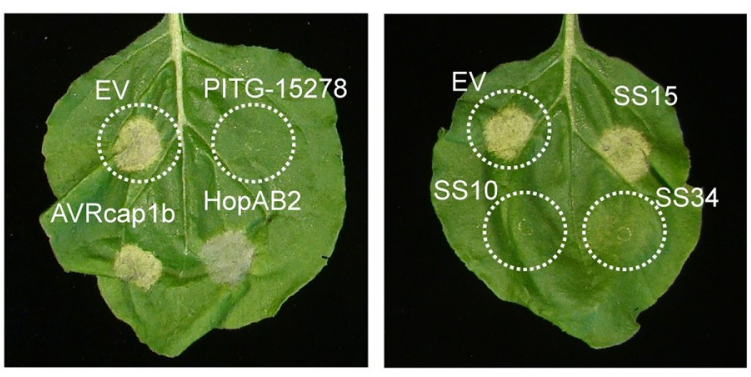

*

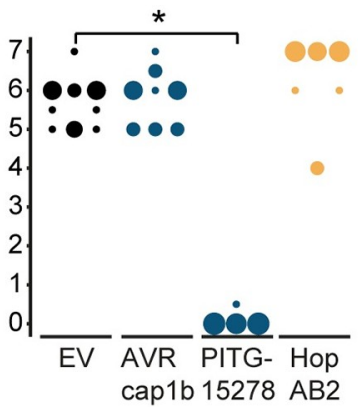

Pathogen species

- Aphid $(n=50)$

- Bacteria $(n=26)$

- Nematode $(n=26)$

- Oomycete $(n=65)$

Fig 1. Five effectors suppress Prf- (NRC2/3 dependent) or Rpi-blb2- (NRC4 dependent) mediated cell death. (A) A schematic representation of cell death assay strategy used. Effectors and EV were transformed into Agrobacterium tumefaciens and transiently coexpressed in N. benthamiana plants with either Pto/ AvrPto or Rpi-blb2/AVRblb2. EV was used as a negative control. HR was scored based on a modified 0-7 scale (Segretin and colleagues) between 5-7 days post-infiltration. (B) A scatterplot of the average HR score of EV versus the average HR score of each tested effector ( $n=165)$; Pto/AvrPto (left panel) Rpi-blb2/ 
AVRblb2 (right panel). Effectors that have suppression activity are represented as outliers within the plot. Results are based on 6 technical replicates (S1-S3 Data). ${ }^{\triangle}$ Two alleles of PITG-15278 (P. infestans strain T30-4 and 17777) suppressed Rpiblb2-mediated cell death. PITG-15278 T30-4 is used as a representative in subsequent experiments. (C) Representative $N$. benthamiana leaves infiltrated with appropriate constructs photographed 5-7 days after infiltration. The R/ AVR pair tested, Prf (Pto/AvrPto) or Rpi-blb2/AVRblb2, are labelled above the leaf panel and effectors labelled on leaf image. (D) HR was scored 5 days postagroinfiltration (S4 Data). The results are presented as a dot plot, where the size of each dot is proportional to the number of samples with the same score (count) within each biological replicate. The experiment was independently repeated 3 times with 6 technical replicates. The columns for either EV or for each individual effector correspond to results from different biological replicates. Dot colours represent pathogen species as indicated in (B). Statistical tests were implemented using the besthr R library [41]. We performed bootstrap resampling tests using a lower significance cutoff of 0.025 and an upper cutoff of 0.975 . Mean ranks of test samples falling outside of these cutoffs in the control samples bootstrap population were considered significant. Significant differences between the conditions are indicated with an asterisk (*). Details of statistical analysis are presented in S2 Fig. AVR, avirulence; EV, empty vector; HR, hypersensitive response.

https://doi.org/10.1371/journal.pbio.3001136.g001

Gpa2, R1, Rx, Bs2, and R8) that were previously assigned to the NRC network [24,25]. We transiently coexpressed AVRcap1b and SS15 with these NRC-dependent sensor NLRs in addition to the previously tested Prf and Rpi-blb2 in N. benthamiana leaves using agroinfiltration. Of these, 5 NLRs were coexpressed with their cognate AVR effectors, whereas we used a constitutively active version of SW5b (SW5 ${ }^{\text {D857V }}$ ). We also included Rpi-vnt1 as an NRC-independent NLR protein negative control [24]. Interestingly, while SW5b was previously shown to signal through NRC2, NRC3, and NRC4 in a redundant manner [24], SW5 $\mathrm{b}^{\mathrm{D} 857 \mathrm{~V}}$ signalled through NRC2 and NRC3 only. These experiments revealed that AVRcap1b and SS15 suppress SW5 ${ }^{\mathrm{D} 857 \mathrm{~V}}$ and Gpa2 in addition to Prf, but none of the other 5 NLRs (Rpi-blb2, R8, R1, Rx, and Bs2). Our findings do, however, contrast to a previous study that showed that SS15 was able to partially suppress Rx-mediated cell death [35]. Additionally, neither AVRcaplb nor SS15 suppressed cell death mediated by the NRC-independent NLR Rpi-vnt1 (Figs 3 and S6 and S6 Data). These results are consistent with the model that AVRcap1b and SS15 suppress NRC2 and NRC 3 but not NRC4, given that, similar to Prf, both SW5 ${ }^{\mathrm{D} 857 \mathrm{~V}}$ and Gpa2 are dependent on NRC2 and NRC3, whereas the other tested NLRs signal through NRC4 specifically or redundantly with NRC2 and NRC3 [24,25].

\section{AVRcaplb and SS15 suppress Rx-mediated cell death only in the absence of NRC4}

The NRC-dependent sensor NLR Rx, which confers extreme resistance to Potato virus $X$ (PVX) by recognising viral coat protein (CP), is dependent on NRC2, NRC3, and NRC4 in a genetically redundant manner [42-45]. The genetic redundancy of NRCs may explain why the Rx-mediated cell death is not suppressed by AVRcap1b and SS15 in N. benthamiana (Fig 3). Since AVRcap1b and SS15 suppress the cell death activity of NRC2 and NRC3 but not NRC4, we reasoned that these 2 effectors should be able to suppress $\mathrm{Rx}$-mediated cell death in the absence of NRC4. To challenge this hypothesis, we knocked down NRCs in Rx-transgenic $N$. benthamiana plants using Tobacco rattle virus (TRV)-induced gene silencing, either individually (TRV:NRC4) or in combination (TRV:NRC2/3) (Fig 4A). Three weeks after inoculation with TRV, we coexpressed AVRcap1b or SS15 with CP in NRC-silenced leaves. These experiments showed that both AVRcap1b and SS15 suppress Rx-mediated cell death in NRC4silenced leaves, but not in NRC2/3-silenced leaves nor in the TRV:EV control (Figs 4B-4E and S7 and S7 Data). These results further validate our earlier finding that AVRcap1b and SS15 can specifically suppress the activities of NRC2 and NRC3 but not NRC4.

\section{AVRcap $1 b$ and SS15 compromise Rx-mediated extreme resistance to Potato virus $X$ in an NRC2- and NRC3- but not NRC4-dependent manner}

We previously showed that cosilencing of $N R C 2, N R C 3$, and $N R C 4$ not only compromised Rxmediated hypersensitive cell death but also abolished extreme resistance to PVX, leading to 
A

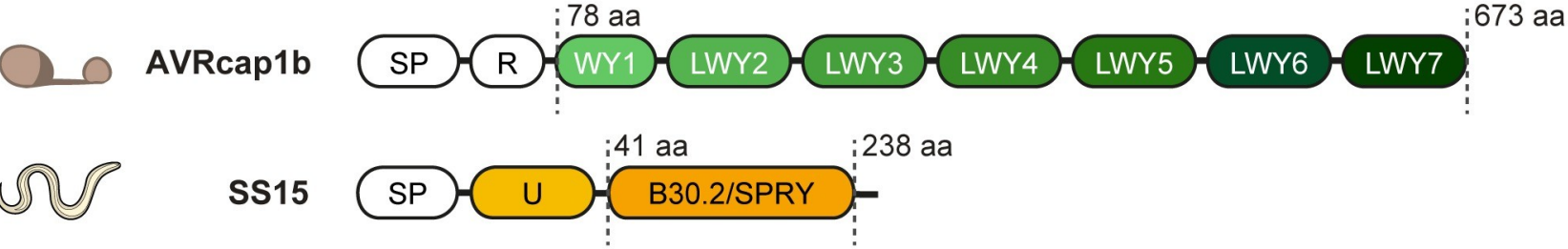

B

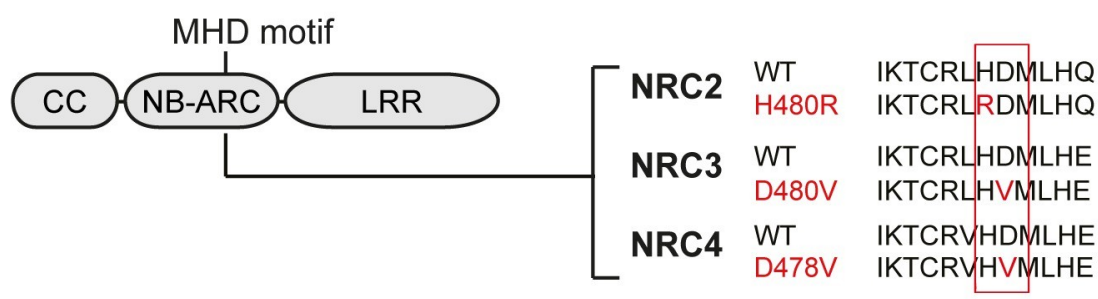

C

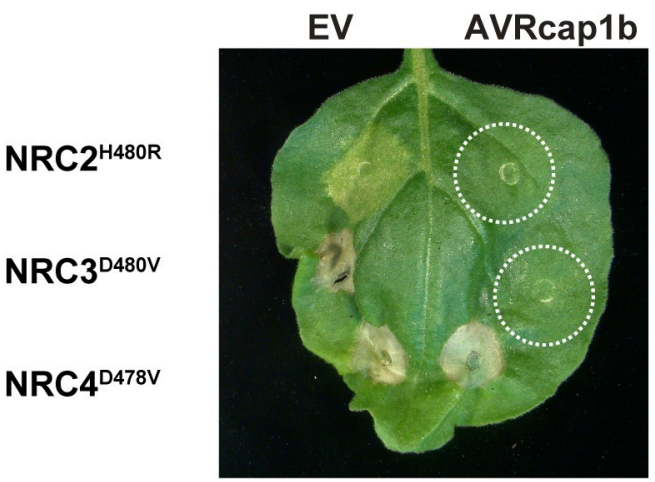

EV

SS15

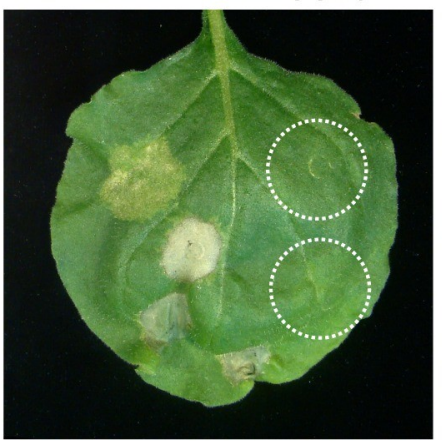

D
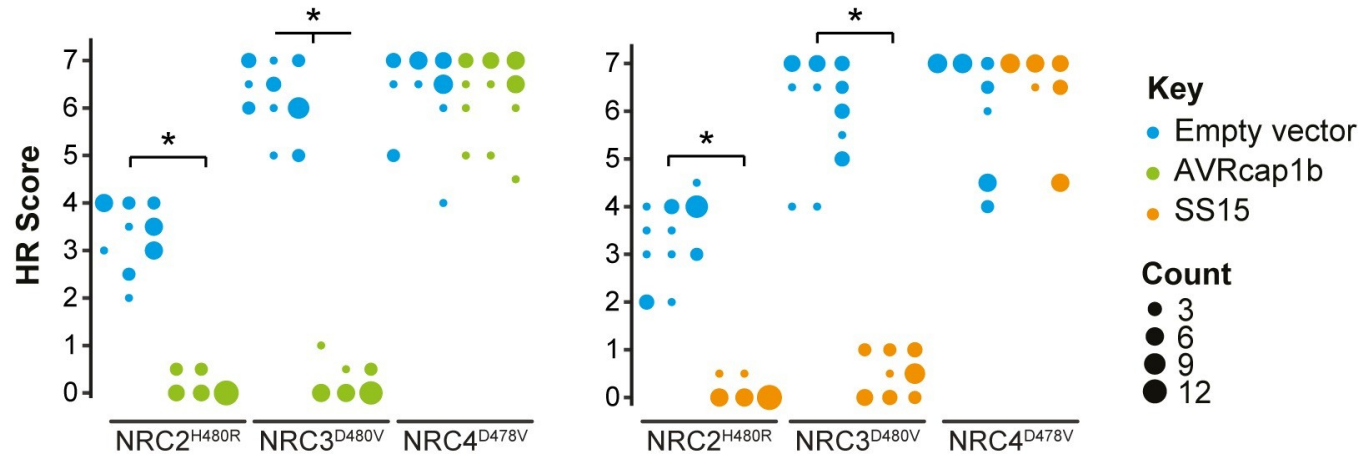

Fig 2. AVRcap1b and SS15 suppress cell death activity mediated by autoactive $\mathrm{NRC}^{\mathrm{H} 480 \mathrm{R}}$ and NRC $3^{\mathrm{D} 480 \mathrm{~V}}$ but not NRC4 ${ }^{\mathrm{D} 478 \mathrm{~V}}$. (A) Domain organisation of the RXLR-WY/LWY domain containing P. infestans effector AVRcap1b and the B30.2/SPRY (IPR001870) domain containing G. rostochiensis effector SS15, where SP = signal peptide, $\mathrm{R}=\mathrm{RxLR}$ motif, $\mathrm{U}=$ uncharacterized domain. (B) Schematic representation of NRC2, NRC3, and NRC4 and the mutated sites in the MHD motif. Substituted residues are shown in red in the multiple sequence alignment. (C) Representative $N$. benthamiana leaves showing HR after coexpression of EV, AVRcap1b, or SS15, indicated above leaf panels, with autoimmune $\mathrm{NRC} 2{ }^{\mathrm{H} 480 \mathrm{R}}, \mathrm{NRC} 3^{\mathrm{D} 480 \mathrm{~V}}$, and $\mathrm{NRC4}^{\mathrm{D} 478 \mathrm{~V}}$ mutants. Plants were photographed 5 days after agroinfiltration. (D) HR was scored 5 days post-agroinfiltration (S5 Data). The results are presented as a dot plot, where the size of each dot is proportional to the number of samples with the same score (count) within each biological replicate. The experiment was independently repeated 3 times each with 6 technical replicates. The columns of each tested condition (labelled on the bottom of the plot) correspond to results from different biological replicates. Statistical tests were implemented using the besthr R library [41]. We performed bootstrap resampling tests using a lower significance cutoff of 0.025 and an upper cutoff of 0.975 . Mean ranks of test samples falling outside of these cutoffs in the control samples bootstrap population were considered significant. Significant differences between the conditions are indicated with an asterisk $\left({ }^{*}\right)$. Details of statistical analysis are presented in S4 Fig. CC, coiled-coil; EV, empty vector; HR, hypersensitive response; LRR, leucine-rich repeat; MHD, methioninehistidine-aspartate; NB-ARC, nucleotide-binding domain shared with APAF-1, various R-proteins, and CED-4; WT, wild type.

https://doi.org/10.1371/journal.pbio.3001136.g002 
A

EV
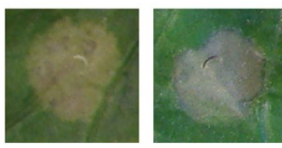

AVRcap1b

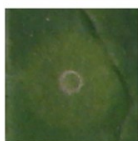

B
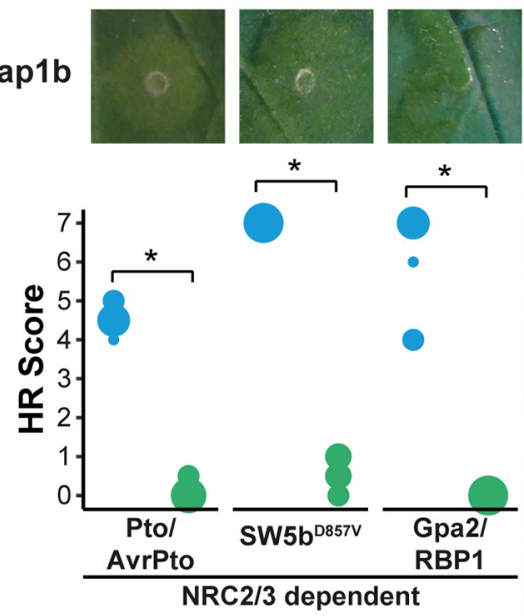

C

D
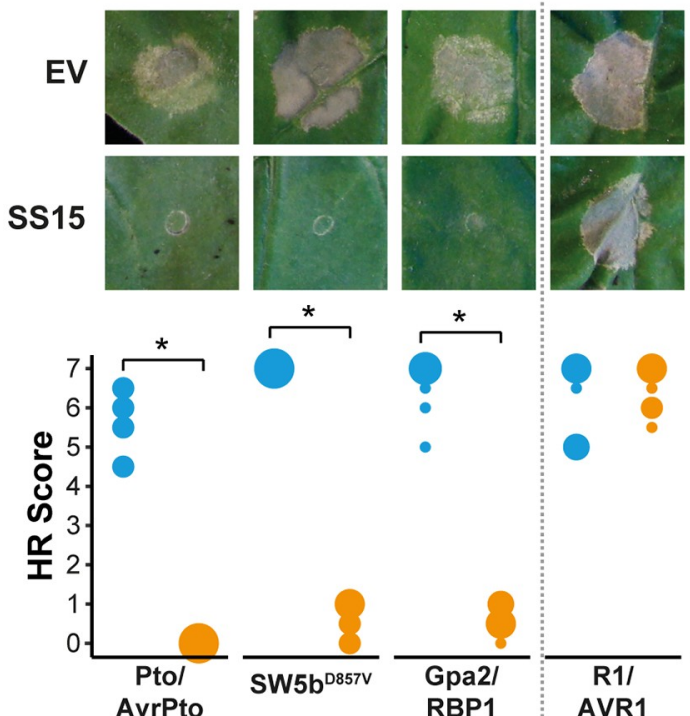

NRC2/3 dependent
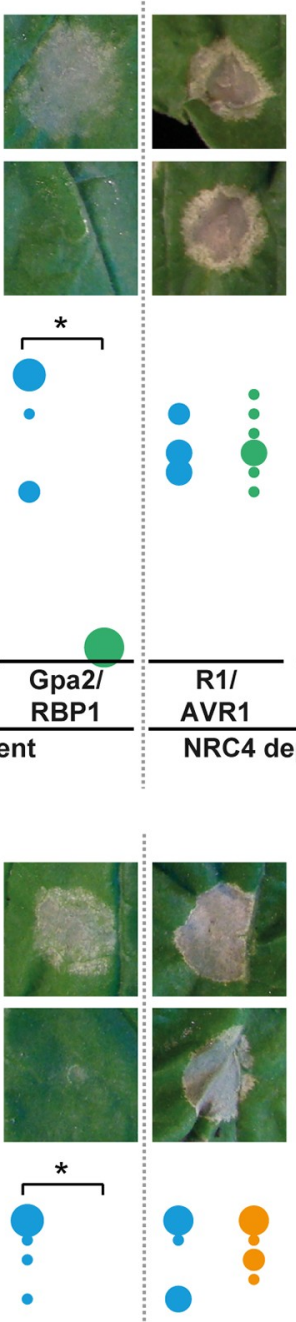
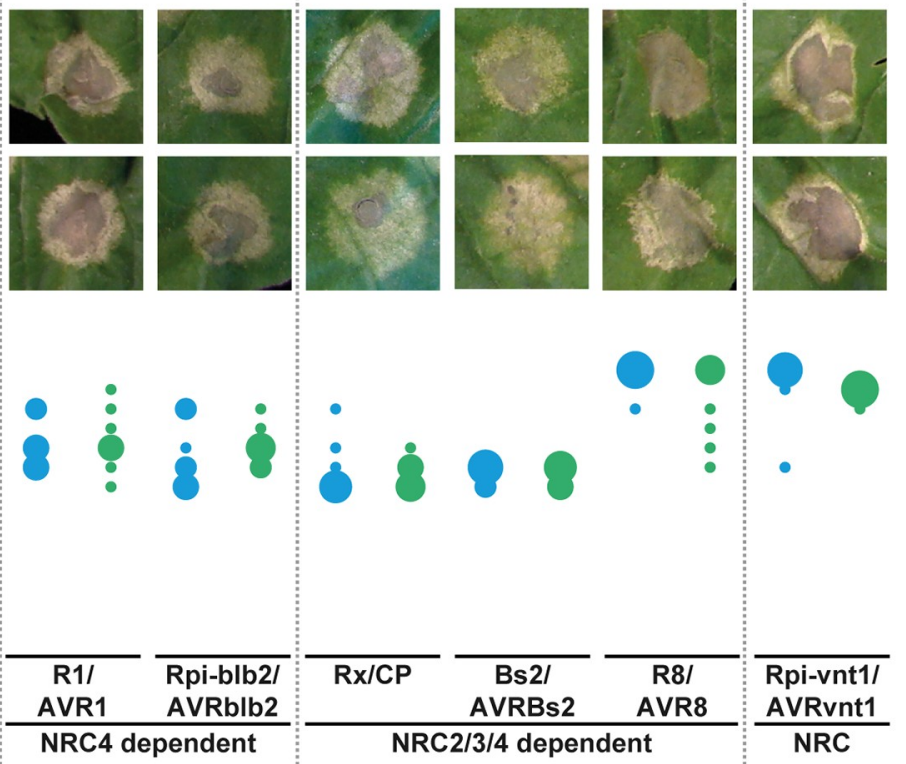

Key

- Empty vector

- AVRcap1b

\section{Count}

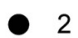

4

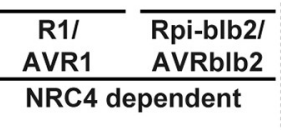

NRC2/3/4 dependent independent
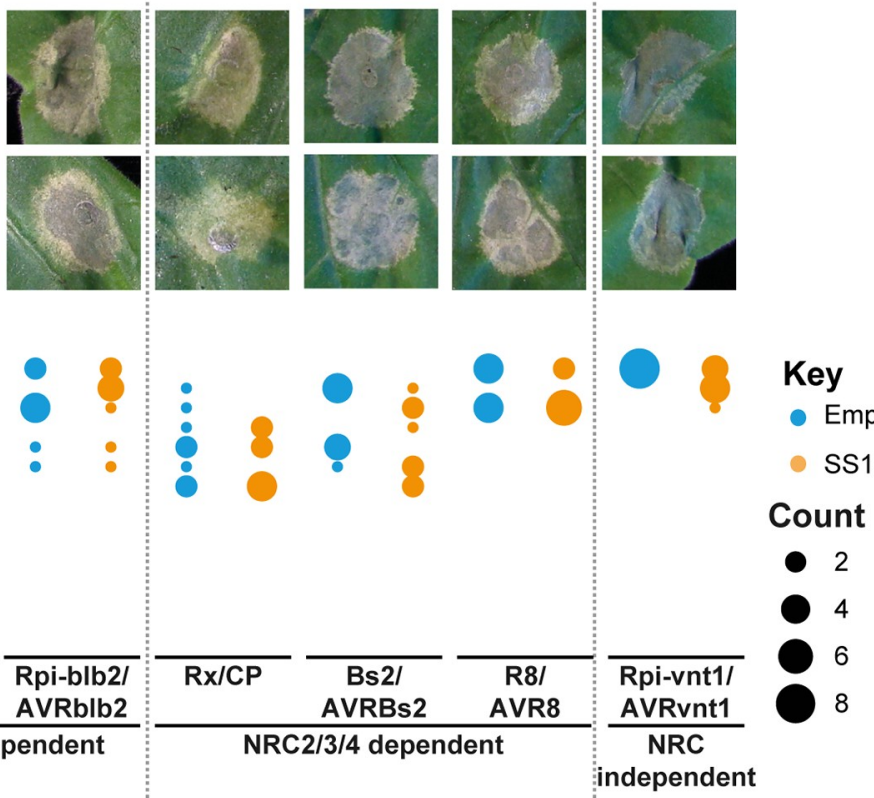

Fig 3. AVRcap1b and SS15 suppress cell death mediated by sensor NLRs that signal through NRC2 and NRC3 but not NRC4. Representative leaf panels showing HR phenotypes of (A) EV and AVRcap1b or (C) EV and SS15 coinfiltrated with a range of sensor NLRs (labelled on the bottom of panels B and D). Photographs were taken 5 days post-agroinfiltration. (B, D) HR results are presented as dot plots, where the size of each dot is proportional to the number of samples with the same score (count). A total of 8 technical replicates were completed for each treatment (S6 Data). Statistical tests were implemented using the besthr R library [41]. We performed bootstrap resampling tests using a lower significance cutoff of 0.025 and an upper cutoff of 0.975 . Mean ranks of test samples falling outside of these cutoffs in the control samples bootstrap population were considered significant. Significant differences between the conditions are indicated with an asterisk $\left(^{*}\right)$. Details of statistical analysis are presented in S6 Fig. EV, empty vector; HR, hypersensitive response; NLR, nucleotide-binding domain and leucine-rich repeat; NRC, NLR required for cell death.

https://doi.org/10.1371/journal.pbio.3001136.g003

trailing necrotic lesions indicative of virus spread [24]. To determine the extent to which suppression mediated by AVRcap1b and SS15 translates into reduced viral infection, we tested the effectors ability to compromise Rx-mediated resistance to PVX. We transiently expressed 
A

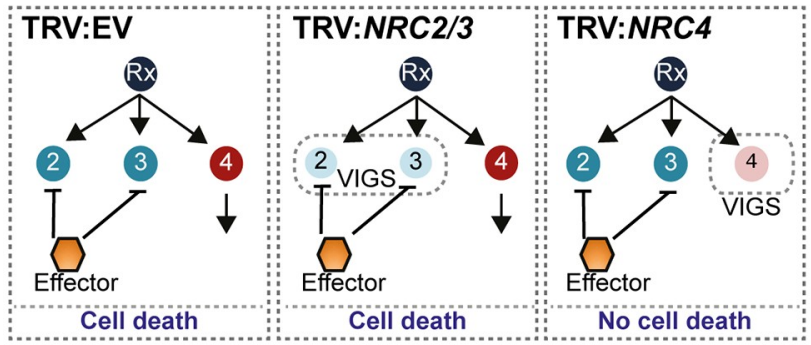

B

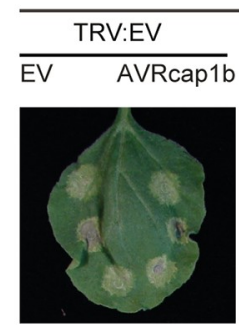

Rx Transgenic TRV:NRC2/3
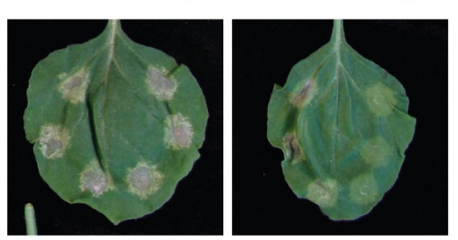

D
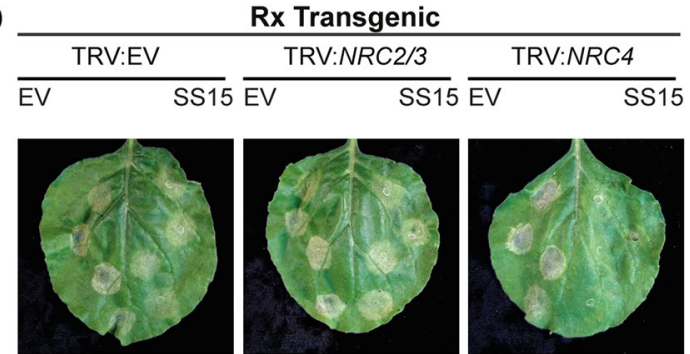

C

Rx Transgenic

$\frac{\text { TRV:EV }}{\text { EV AVRcap1b }} \frac{\text { TRV:NRC2/3 }}{\text { EV AVRcap1b }} \frac{\text { TRV:NRC4 }}{\text { EV AVRcap1b }}$

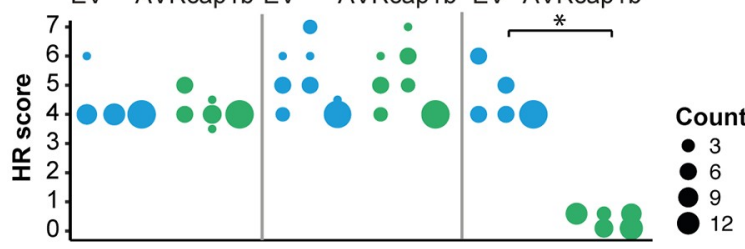

E

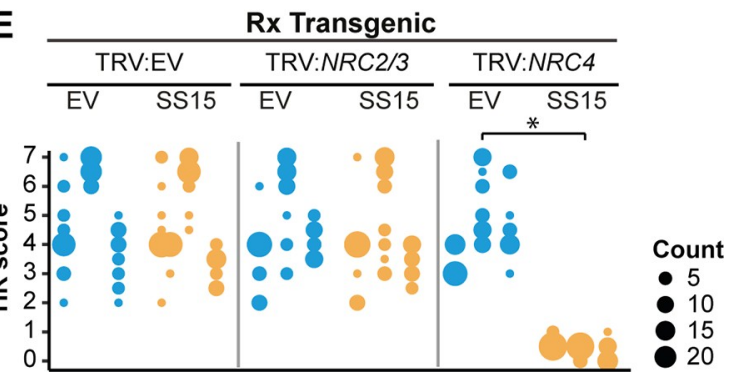

Fig 4. AVRcap1b and SS15 suppress Rx-mediated cell death in NRC4-silenced Rx-transgenic Nicotiana benthamiana plants. (A) A schematic representation of the silencing and infiltration strategy undertaken. Photo of representative N. benthamiana leaves showing HR after coexpression of (B) EV or AVRcap1b and (D) EV or SS15 in TRV:EV, TRV:NRC2/3, and TRV:NRC4 silenced Rx-transgenic $N$. benthamiana plants, where EV was used as a negative control. Silencing treatments (TRV:EV, TRV:NRC2/3, and TRV:NRC4) are indicated above leaf panels. Plants were photographed 5 days after agroinfiltration. Cell death response was scored 5 days after agroinfiltration (S7 Data) (C) EV and AVRcap1b and (E) EV and SS15 and presented as dot plots, where dot colour represents either EV (blue), AVRcap1b (green), or SS15 (orange) and dot size is proportional to the number of samples with the same score (count). The experiment was independently repeated 3 times. Each replicate is represented by different columns within each silencing treatment for either EV, AVRcap1b, or SS15. Statistical tests were implemented using the besthr R library [41]. We performed bootstrap resampling tests using a lower significance cutoff of 0.025 and an upper cutoff of 0.975 . Mean ranks of test samples falling outside of these cutoffs in the control samples bootstrap population were considered significant. Significant differences between the conditions are indicated with an asterisk $\left({ }^{*}\right)$. Details of statistical analysis are presented in S7 Fig. EV, empty vector; HR, hypersensitive response; VIGS, virus-induced gene silencing.

https://doi.org/10.1371/journal.pbio.3001136.g004

AVRcap1b or SS15 in Rx-transgenic N. benthamiana plants that were silenced for NRCs either individually (TRV:NRC4) or in combination (TRV:NRC2/3, TRV:NRC2/3/4). We then infected the leaves by agroinfection with Agrobacterium tumefaciens carrying PVX (pGR106:: PVX::GFP) using a toothpick inoculation method and documented the formation of trailing necrotic lesions at the inoculated spots (see PVX infection assays (agroinfection)) $[24,43,46]$ (Fig 5A). Consistent with previous experiments [24], we observed trailing necrosis in the NRC triple silenced Rx leaves (TRV:NRC2/3/4) regardless of the presence or absence of AVRcap1b or SS15 (Fig 5B and 5C, S8 and S9 Data). We also observed trailing necrosis when AVRcap1b or SS15 was expressed in NRC4-silenced (TRV:NRC4) Rx leaves, indicating that both effectors compromised Rx-mediated resistance when NRC4 is depleted (Fig 5B and 5C, S8 and S9 Data). In contrast, Rx-mediated resistance to PVX was not compromised by AVRcap1b or 
A

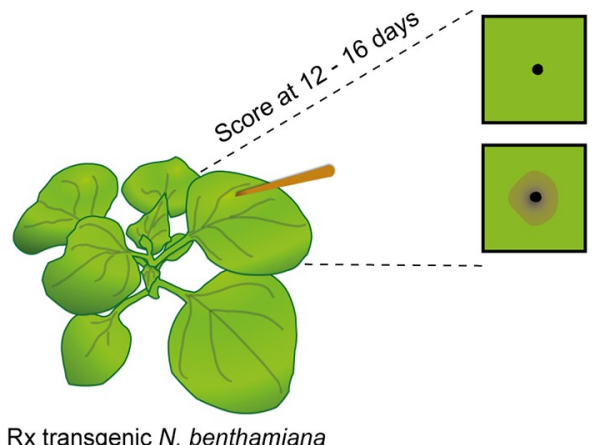

No necrosis $=$ Extreme resistance

B

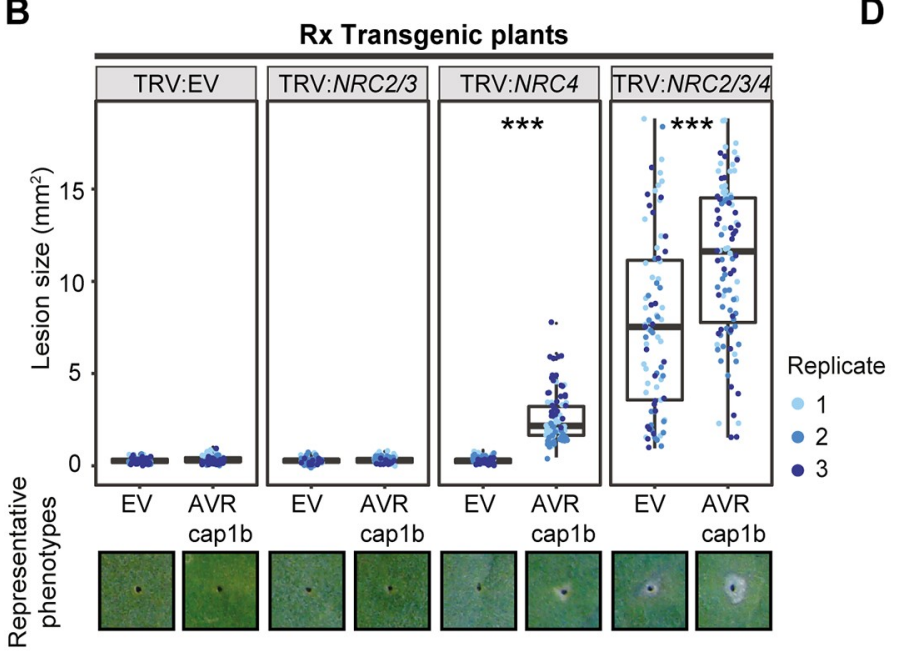

\section{C}

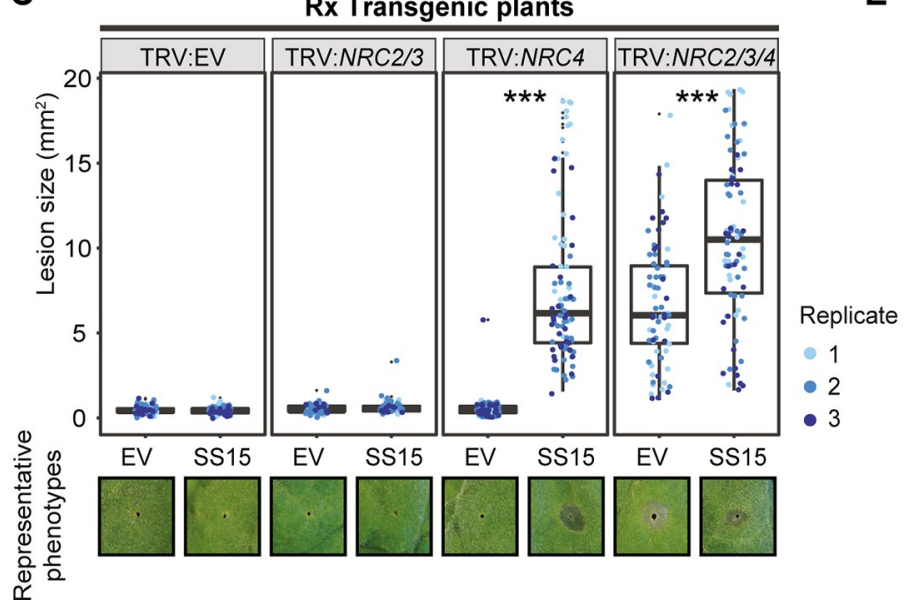

D

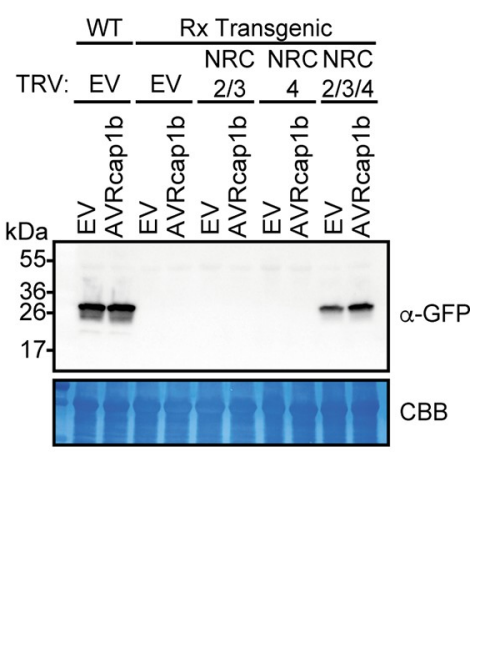

E

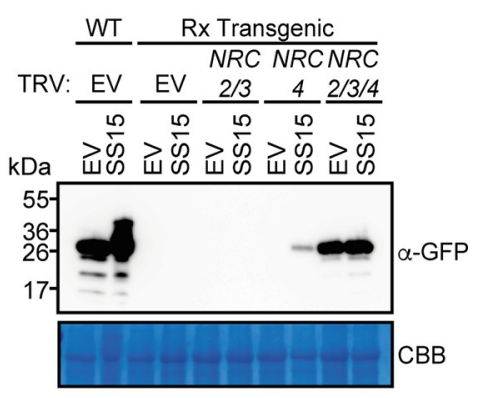

Fig 5. AVRcap1b and SS15 compromise Rx-mediated extreme resistance to PVX in NRC4-silenced plants. (A) Toothpick inoculation method previously described by $\mathrm{Wu}$ and colleagues allowed examination of the spread of trailing necrotic lesions due to the partial resistance mediated by Rx. EV, NRC2, NRC3, or NRC4 were silenced individually or in combination in Rx-transgenic N. benthamiana plants by TRV. (B) EV or AVRcap1b and (C) EV or SS15 were expressed, via agroinfiltration, in leaves of the NRC-silenced plants 1 day before PVX inoculation. PVX-GFP (pGR106-GFP) was inoculated using the toothpick inoculation method, as per panel (A). Photographs were taken 12-16 days after PVX inoculation. The size of the necrotic lesions was measured using Fiji (previously ImageJ) (S8 Data). Data acquired from different biological replications are presented in different colours. Statistical differences among the samples were analysed with mixed model ANOVA and a Tukey HSD test ( $p$-value $<0.01$ ), where the fixed effect is the silencing treatment (TRV: EV, TRV:NRC2/3, TRV:NRC4, TRV:NRC2/3/4) with either EV or AVRcap1b and the random effect is the experimental replicate. Significant differences between the conditions are indicated with asterisks $\left({ }^{* * *}, p\right.$-value $<0.0001$ ) (S9 Data). 
Representative phenotypes observed for each treatment are presented below the boxplots. Immunoblot analysis of GFP accumulation of pGR106-GFP toothpick inoculated sites in the presence of (D) AVRcap1b and (E) SS15 in Rx-transgenic or WT N. benthamiana plants. AVR, avirulence; CBB, Coomassie brilliant blue; EV, empty vector; HSD, honestly significant difference; NRC, NLR required for cell death; PVX, Potato virus X; TRV, Tobacco rattle virus; WT, wild type.

https://doi.org/10.1371/journal.pbio.3001136.g005

SS15 in NRC2/3-silenced (TRV:NRC2/3) leaves. Virus accumulation in the different treatments was validated by western blot detection of GFP protein driven by the subgenomic promoter of PVX::GFP (Fig 5D and 5E). Perturbation of PVX resistance was more markedly affected by SS15 compared to AVRcap1b based on lesion size and PVX accumulation (Fig 5C$5 \mathrm{E})$. These results indicate that the AVRcap $1 \mathrm{~b}$ and SS15 effectors not only suppress NLRmediated cell death but also counteract the disease resistance phenotype mediated through NRC2 and NRC3.

\section{Yeast two-hybrid screens reveal candidate host interactors of the AVRcap1b and SS15 effectors}

To investigate how AVRcap1b and SS15 suppress NRC activities, we set out to identify their host interactors. We used the effectors as baits in unbiased yeast two-hybrid (Y2H) screens against a N. benthamiana-mixed tissue cDNA library (ULTImate Y2H, Hybrigenics Services, Paris, France). AVRcap1b was screened against a combined approximately 140 million clones, which resulted in 13 candidate interacting proteins from a total of 35 positive clones (S2 Table). SS15 was screened against approximately 61 million clones resulting in 10 candidate proteins from a total of 202 positive clones (S3 Table). Remarkably, NRC3 and NRC4 were among the recovered SS15 protein interactors, which is notable given that NLR proteins are rarely recovered from $\mathrm{Y} 2 \mathrm{H}$ screens. The NRC3 and NRC4 fragments that were recovered from the $\mathrm{Y} 2 \mathrm{H}$ screen matched the CC-NB-ARC domains indicating that $\mathrm{SS} 15$ may bind the $\mathrm{N}$-terminal half of NRC proteins (Fig 6A, S3 Table). NRC2 was not recovered as an interactor in the $\mathrm{Y} 2 \mathrm{H}$ screen for SS15; however, we cannot rule out that this might be due to poor accumulation of this NLR or its subdomains in the yeast strains used.

To further investigate the interactions between SS15 and NRCs, we sought out to delimit the binding domain within the NLRs. Since the prey fragment hits from the $\mathrm{Y} 2 \mathrm{H}$ screen covered the CC-NB-ARC domain of NRC3 and NRC4 (Fig 6A), we generated CC and NB-ARC domain truncations and tested them in additional $\mathrm{Y} 2 \mathrm{H}$ experiments for interaction with SS15. These assays revealed that SS15 binds to the NB-ARC but not the CC domains of NRC2, NRC3, and NRC4 (S8 Fig). Consistent with earlier observations, AVRcaplb did not interact with either the CC or NB-ARC domains of the NRCs in these Y2H experiments (S8 Fig).

\section{Unlike AVRcap1b, SS15 associates with NRC2 and NRC3 in planta}

The $\mathrm{Y} 2 \mathrm{H}$ results prompted us to investigate the association between AVRcap1b, SS15, and NRCs using co-immunoprecipitation (coIP) of proteins expressed in N. benthamiana, which should reveal the association between the effectors and full-length NRC proteins in a more physiologically relevant condition. To achieve this, we coexpressed each of AVRcap1b::6xHA and 4xHA::SS15 with NRC2::4xMyc, NRC3::4xMyc, and NRC4::4xMyc in N. benthamiana leaves using agroinfiltration and subjected the protein extracts to anti-Myc immunoprecipitation and western blot analyses. These experiments revealed that SS15 co-immunoprecipitated with NRC2 and NRC3, but not with NRC4 (Fig 6B). While we did detect association between NRC4 and SS15 in Y2H, we could only detect a weak NRC4 signal in some biological replicates, suggesting that SS15 may also associate with NRC4 in planta but with markedly lower 
A

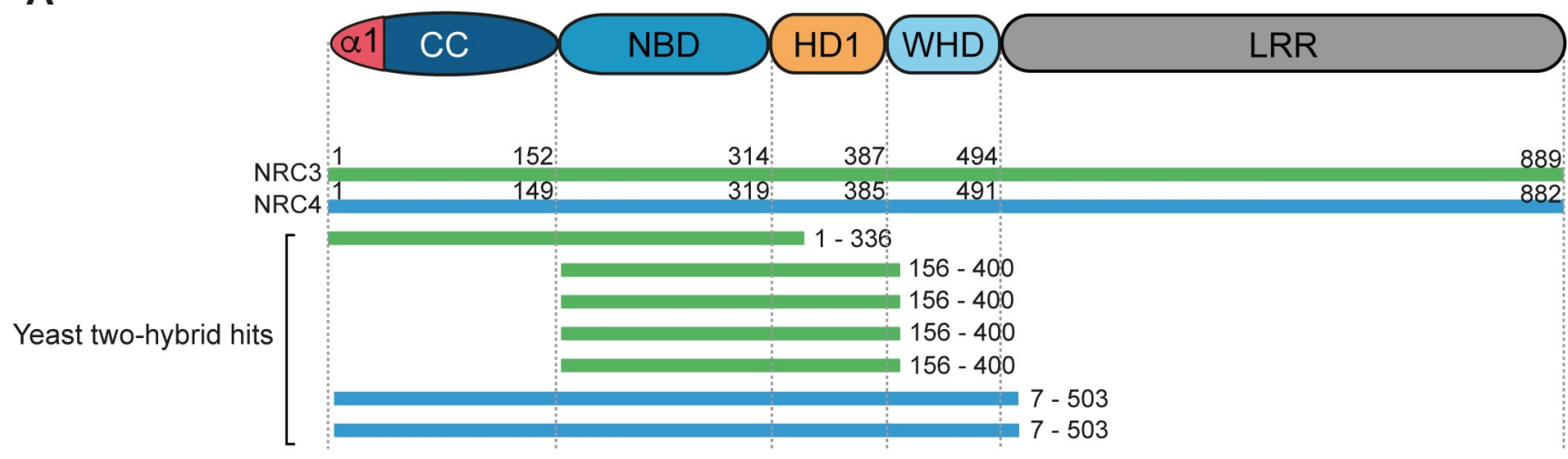

B

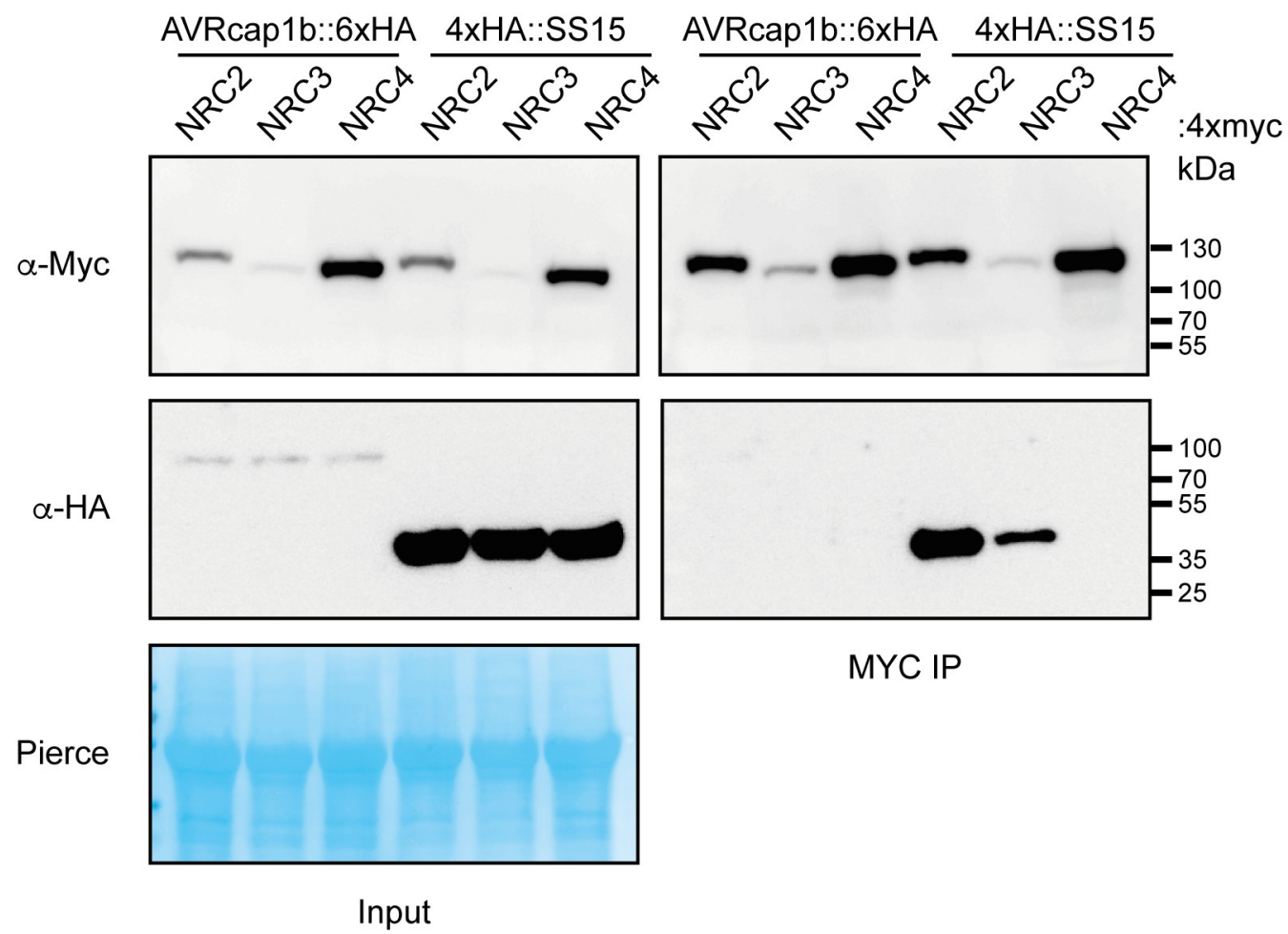

Fig 6. SS15, but not AVRcap1b, associates with NRCs in planta. (A) Schematic diagram of domain organisation of NRC proteins, showing Y2H hits from the Hybrigenics services screen (S3 Table). (B) CoIP experiment of C-terminally 4xmyc-tagged NRC2, NRC3, and NRC4 with C-terminally HAtagged AVRcap1b::6xHA and N-terminally tagged 4xHA:SS15 (labelled above). Proteins obtained by coIP with MYC beads (MYC IP) and total protein extracts (input) were immunoblotted with the appropriate antisera labelled on the left. Approximate molecular weights ( $\mathrm{kDa}$ ) of the proteins are shown on the right. Rubisco loading control was carried out using Pierce staining. The experiment was performed more than 3 times under different pulldown conditions with similar results. CC, coiled-coil; coIP, co-immunoprecipitation; HD1, helical domain 1; LRR, leucine-rich repeat; NBD, nucleotide-binding domain; NRC, NLR required for cell death; WHD, winged helix domain; $\mathrm{Y} 2 \mathrm{H}$, yeast two-hybrid.

https://doi.org/10.1371/journal.pbio.3001136.g006

affinity compared to NRC2 and NRC3 (S9 Fig). This result aligns with our observation that SS15 is unable to suppress NRC4 in cell death assays. In the case of AVRcap1b, the coIP experiments were consistent with the $\mathrm{Y} 2 \mathrm{H}$ screens as we did not detect an association between AVRcaplb and any of the 3 NRCs (Fig 6B). 


\section{SS15 directly binds the NB-ARC domain of NRC3 in vitro}

To further examine the association between SS15 and the NB-ARC domains of NRCs, we purified these proteins for in vitro assays using Escherichia coli as a heterologous expression system. We successfully obtained homogeneous SS15 and NRC3 NB-ARC domain $\left(\mathrm{NRC}^{\mathrm{NB}-\mathrm{ARC}}\right.$ ) proteins but were unable to obtain purified NRC2 NB-ARC or NRC4 NB-ARC domains due to solubility and stability issues. We subjected purified SS15 and NRC ${ }^{\text {NB-ARC }}$ to gel filtration and found that these proteins elute at $239 \mathrm{ml}$ and $227 \mathrm{ml}$, which correspond to 42.52 and $27.75 \mathrm{kDa}$, respectively (Fig 7). To determine whether the 2 proteins form a complex in vitro, we mixed cells expressing individual proteins and copurified SS15 and NRC $3^{\text {NB-ARC }}$ for gel filtration assays (see Protein-protein interaction studies: Protein purification from $E$. coli and in vitro protein-protein interaction studies). The copurified mixture of SS15 and NRC $3^{\mathrm{NB}-\mathrm{ARC}}$ resulted in a peak shift with an elution volume at $211 \mathrm{ml}$, which corresponds to $84.02 \mathrm{kDa}$. Further validation by SDS-PAGE of the fractions under the new peak confirmed the presence of both proteins (Fig 7). In our gel filtration assays, the protein molecular weight calibration led to overestimates of the predicted molecular masses of the proteins, both alone and in complex (NRC3 $3^{\mathrm{NB}-\mathrm{ARC}}$ is $41 \mathrm{kDa}$, SS15 is $24.5 \mathrm{kDa}$, and NRC $3^{\mathrm{NB}-\mathrm{ARC}}-\mathrm{SS} 15$ complex is $65.5 \mathrm{kDa}$ ). However, the results indicate that monomeric forms of each state exist in solution and that the 2 proteins probably enter in a 1:1 complex under these experimental conditions. Taken together, these results suggest that SS15 forms a complex with $\mathrm{NRC}^{\mathrm{NB}-\mathrm{ARC}}$ in vitro.

\section{SS15 associates with p-loop mutants of NRC2 and NRC3}

The p-loop motif within the NB-ARC domain of NLR proteins is crucial for ATP binding and hydrolysis, a biochemical step that is essential for NLR oligomerisation and activation [47]. To determine whether SS15 associates with p-loop mutants of the NRCs, we performed coIP experiments in $N$. benthamiana with the NRC p-loop mutants $\mathrm{NRC} 2{ }^{\mathrm{K} 188 \mathrm{R}}, \mathrm{NRC} 3^{\mathrm{K} 191 \mathrm{R}}$, and $\mathrm{NRC}^{\mathrm{K} 190 \mathrm{R}}$ [24]. These experiments revealed that SS15 associates with the p-loop mutants of $\mathrm{NRC} 2\left(\mathrm{NRC} 2^{\mathrm{K} 188 \mathrm{R}}\right.$ ) and NRC3 (NRC3 ${ }^{\mathrm{K} 191 \mathrm{R}}$ ) (S10 Fig). Since an intact p-loop is not required for SS15-NRC association, our findings suggest that SS15 can probably enter in complex with inactive forms of NRC2 and NRC3.

\section{SS15 associates with activated forms of NRC2}

We next investigated the extent to which SS15 associates with activated NRC2 and NRC3. We coexpressed 4xHA::SS15 with C-terminally $4 \mathrm{xMyc}$-tagged autoimmune forms of $\mathrm{NRC}^{\mathrm{H} 480 \mathrm{R}}$ and $\mathrm{NRC} 3{ }^{\mathrm{D} 480 \mathrm{~V}}$ in $\mathrm{N}$. benthamiana using agroinfiltration and subjected the protein extracts to anti-Myc immunoprecipitation and western blot analyses. We found that SS15 co-immunoprecipitated with $\mathrm{NRC} 2{ }^{\mathrm{H} 480 \mathrm{R}}$, indicating that this effector associates with activated forms of NRC2 (Fig 8). Our results for $\mathrm{NRC}^{\mathrm{D} 480 \mathrm{~V}}$, however, were inconclusive, since this protein displayed poor accumulation and could not be detected in western blot analyses under these conditions. Despite this, our overall conclusion is that SS15 binds both inactive and activated forms of NRC2.

\section{AVRcap1b associates with the Nicotiana benthamiana ENTH/VHS-GAT domain protein TOL9a in planta}

Given that AVRcaplb did not interact with NRCs, we reasoned that this effector targets another host protein that is involved in NLR immunity. To narrow down the list of 13 candidate interactors obtained from the $\mathrm{Y} 2 \mathrm{H}$ screen, we subjected AVRcaplb to in planta coIP coupled with tandem mass spectrometry (IP-MS) using methods that are well established in our 


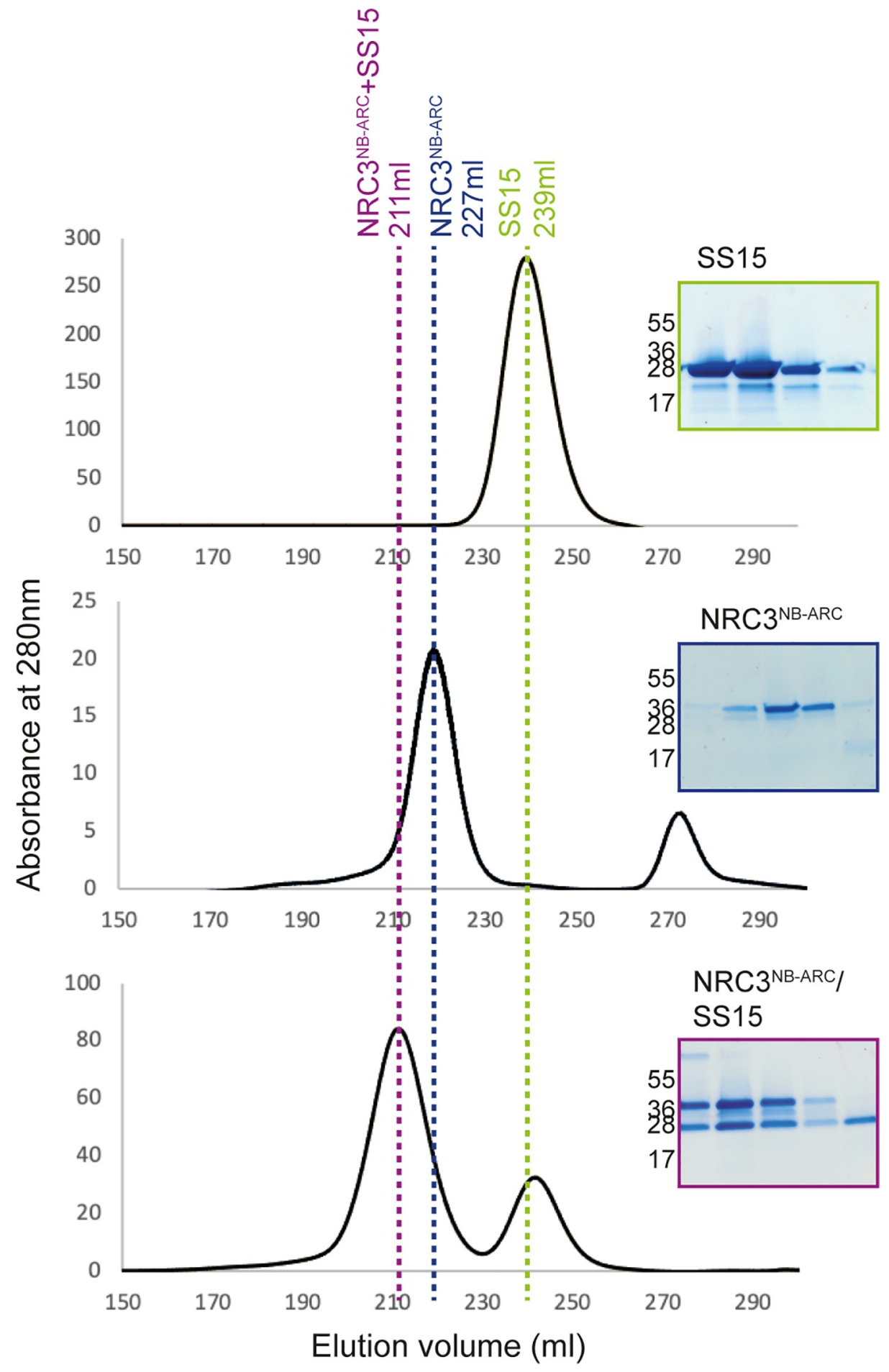

Fig 7. SS15 binds the NB-ARC domain of NRC proteins in vitro. SS15 binds the NRC3 ${ }^{\mathrm{NB}-\mathrm{ARC}}$ domain in vitro. Gel filtration traces obtained for SS15 (top), NRC $3^{\mathrm{NB}-\mathrm{ARC}}$ domain (middle), and a 1:1 mixture of the complex (bottom). Insets show SDS-PAGE gels of the fractions collected across the elution peaks.

https://doi.org/10.1371/journal.pbio.3001136.g007

laboratory [48-50]. IP-MS experiments with GFP::AVRcap1b resulted in the identification of 8 unique AVRcap1b interactors that were not recovered with the control GFP::PexRD54, another P. infestans effector of a similar fold and size (S11 Fig, S4 Table). Three of the 8 


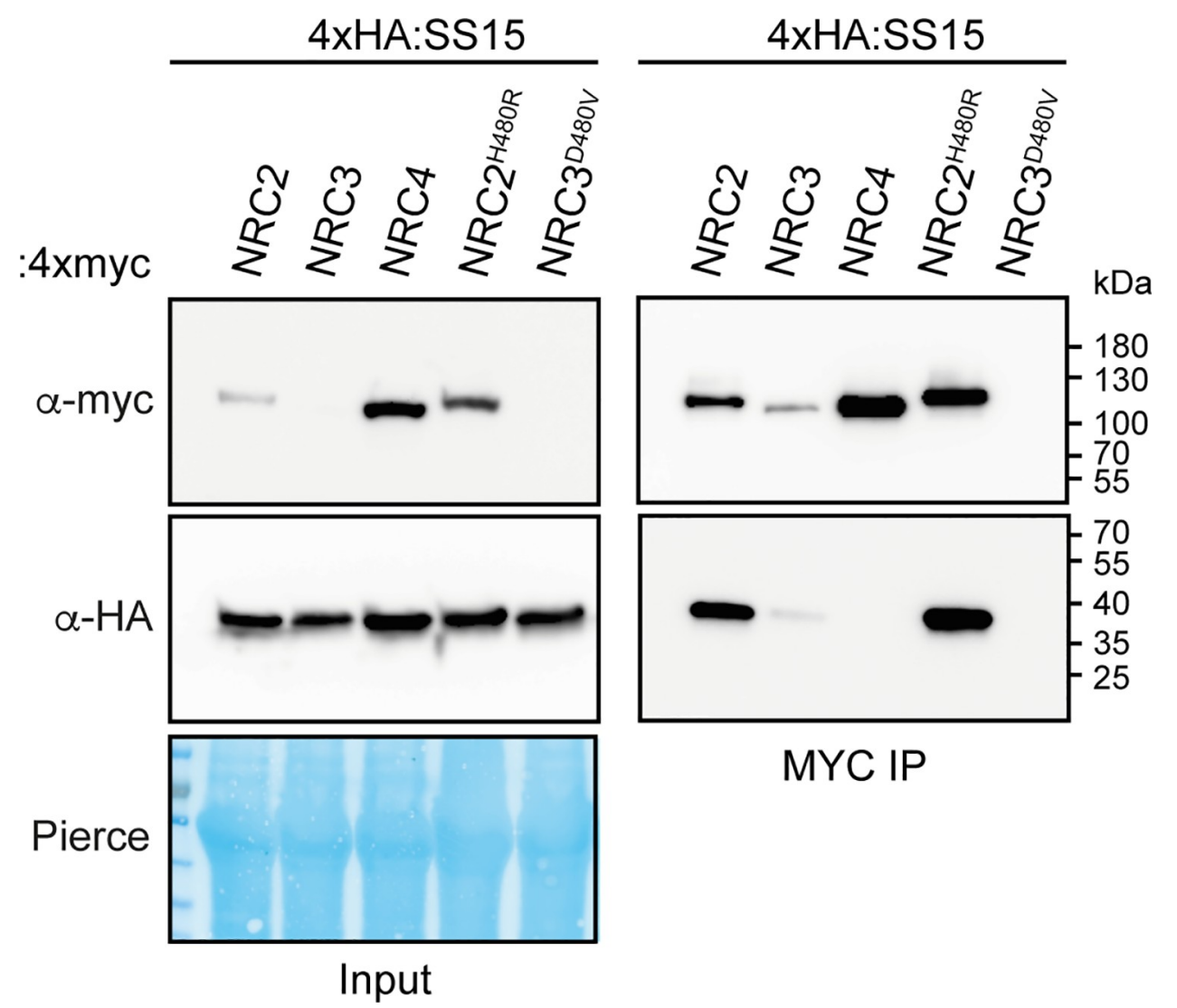

Fig 8. SS15 associates with autoactive NRC2. N-terminally 4xHA-tagged SS15 was transiently coexpressed in N. benthamiana with C-terminally $4 x$ Myc tagged-NRC2, NRC3, NRC4, $\mathrm{NRC}^{\mathrm{H} 480 \mathrm{R}}, \mathrm{NRC} 3^{\mathrm{D} 480 \mathrm{~V}}$, and $\mathrm{NRC} 4^{\mathrm{D} 478 \mathrm{~V}}$. IP was performed with agarose beads conjugated to $\mathrm{Myc}$ (Myc IP) antibodies. Total protein extracts (Input) and proteins obtained by coIP were immunoblotted with appropriate antisera labelled on the right. Approximate molecular weights $(\mathrm{kDa})$ of the proteins are shown on the right. Rubisco loading controls were conducted using Pierce staining. This experiment is representative of 2 independent replicates. coIP, co-immunoprecipitation; IP, immunoprecipitation.

https://doi.org/10.1371/journal.pbio.3001136.g008

interactors were different members of the Target of Myb 1-like protein (TOL) family of ENTH/VHS-GAT domain-containing proteins that function in membrane trafficking as part of the endosomal sorting complex required for transport (ESCRT) pathway [51,52] (Figs 9A and S10 and S4 Table). One of these candidate TOLs, Nbv6.1trP4361, was independently recovered in the $\mathrm{Y} 2 \mathrm{H}$ screen ( $\mathrm{S} 2 \mathrm{Table}$ ). Therefore, we decided to further investigate the corresponding protein (hereafter referred to as NbTOL9a) as a candidate host target of AVRcaplb.

Computational analyses of the N. benthamiana genome revealed 4 TOL paralogs in addition to NbTOL9a, which we termed NbTOL9b (Nbv6.1trP9166), NbTOL3 (Nbv6.1trA40123), NbTOL6 (Nbv6.1trP73492), and NbTOL9c (Nbv6.1trA64113) following previously published nomenclature (S5 Table, S12 Fig) [53]. To validate the association between AVRcap1b and NbTOL proteins, we coexpressed GFP::AVRcap $1 \mathrm{~b}$ with C-terminally 6xHA-tagged fusions of the 5 TOL paralogs, in N. benthamiana leaves, and performed anti-GFP and anti-HA immunoprecipitations. AVRcap1b associated with NbTOL9a and, to a lesser extent, with NbTOL9b and NbTOL9c in the GFP pulldown (GFP IP). However, AVRcap1b only associated with NbTOL9a in the reciprocal HA pulldown (HA IP) (Fig 9B). In both experiments, NbTOL9a protein did not associate with the negative control GFP::PexRD54. These results indicate that AVRcaplb associates with members of the NbTOL family, exhibiting a stronger affinity for NbTOL9a. Based on this conclusion, we focused subsequent experiments on NbTOL9a. 
A

Target of Myb 1-like protein 9a (NbTOL 9a - Nbv6.1trP4361)

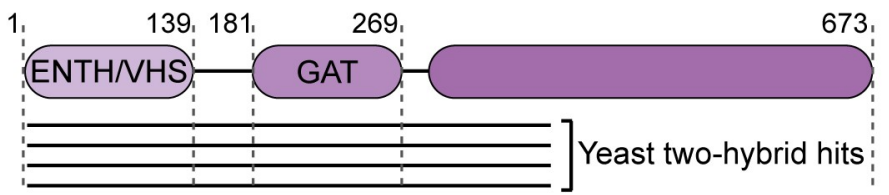

B

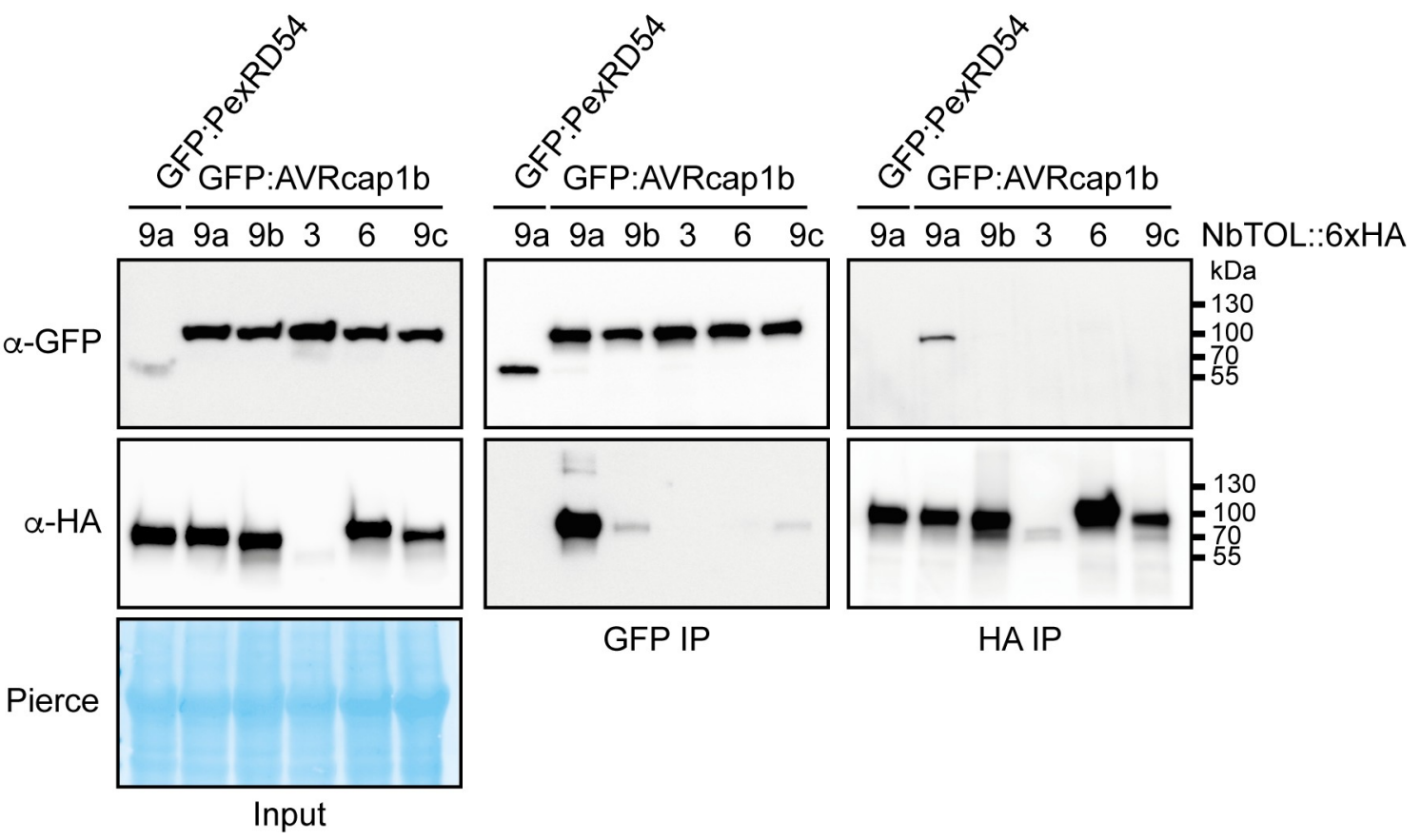

Fig 9. AVRcap1b associates with NbTOL9a in planta. (A) Schematic diagram of domain organisation of NbTOL9a, showing Y2H hits from the screen (S2 Table). (B) CoIP experiment between AVRcap1b and 5 NbTOL family proteins (NbTOL9a, NbTOL9b, NbTOL3, NbTOL6, and NbTOL9c). N-terminally GFP-tagged AVRcap1b was transiently coexpressed with all 5 NbTOL proteins fused to a Cterminal 6xHA tag. N-terminally GFP-tagged PexRD54 was used as a negative control. IP were performed with agarose beads conjugated to either GFP (GFP-IP) or HA (HA-IP) antibodies. Total protein extracts were immunoblotted with appropriate antisera labelled on the left. Approximate molecular weights $(\mathrm{kDa})$ of the proteins are shown on the right. Rubisco loading controls were conducted using Pierce staining. This experiment is representative of 3 independent replicates. coIP, co-immunoprecipitation; IP, immunoprecipitation; NbTOL, N. benthamina Target of Myb 1-like protein; Y2H, yeast two-hybrid.

https://doi.org/10.1371/journal.pbio.3001136.g009

\section{NbTOL9a negatively modulates the cell death triggered by NRC2 and NRC3 but not NRC4 or NbZAR1}

To gain additional insights into the role of NbTOL9a in NRC-mediated hypersensitive cell death, we altered NbTOL9a expression in N. benthamiana. First, we investigated the effect of silencing $\mathrm{NbTOL9a}$ on NRC autoimmunity. We generated a hairpin-silencing construct (RNAi::NbTOL9a) that mediates silencing of NbTOL9a in transient expression assays in $N$. benthamiana leaves (S13 Fig). We then coexpressed RNAi::NbTOL9a with $\mathrm{NRC} 2^{\mathrm{H} 480 \mathrm{R}}$ and $\mathrm{NRC} 3^{\mathrm{D} 480 \mathrm{~V}}$ using agroinfiltration of $N$. benthamiana leaves to test the degree to which silencing of NbTOL9a affects NRC2- and NRC3-mediated cell death. To improve robustness of the assay, we used increasing concentrations of $A$. tumefaciens expressing $\mathrm{NRC}^{\mathrm{H} 480 \mathrm{R}}$ and $\mathrm{NRC}^{\mathrm{D} 480 \mathrm{~V}}\left(\mathrm{OD}_{600}=0.1,0.25\right.$, or 0.5$)$. Silencing of $N b T O L 9 a$ at all tested $\mathrm{OD}_{600}$ concentrations enhanced the cell death response triggered by $\mathrm{NRC} 2^{\mathrm{H} 480 \mathrm{R}}$ and $\mathrm{NRC} 3^{\mathrm{D} 480 \mathrm{~V}}$ but did not 
A
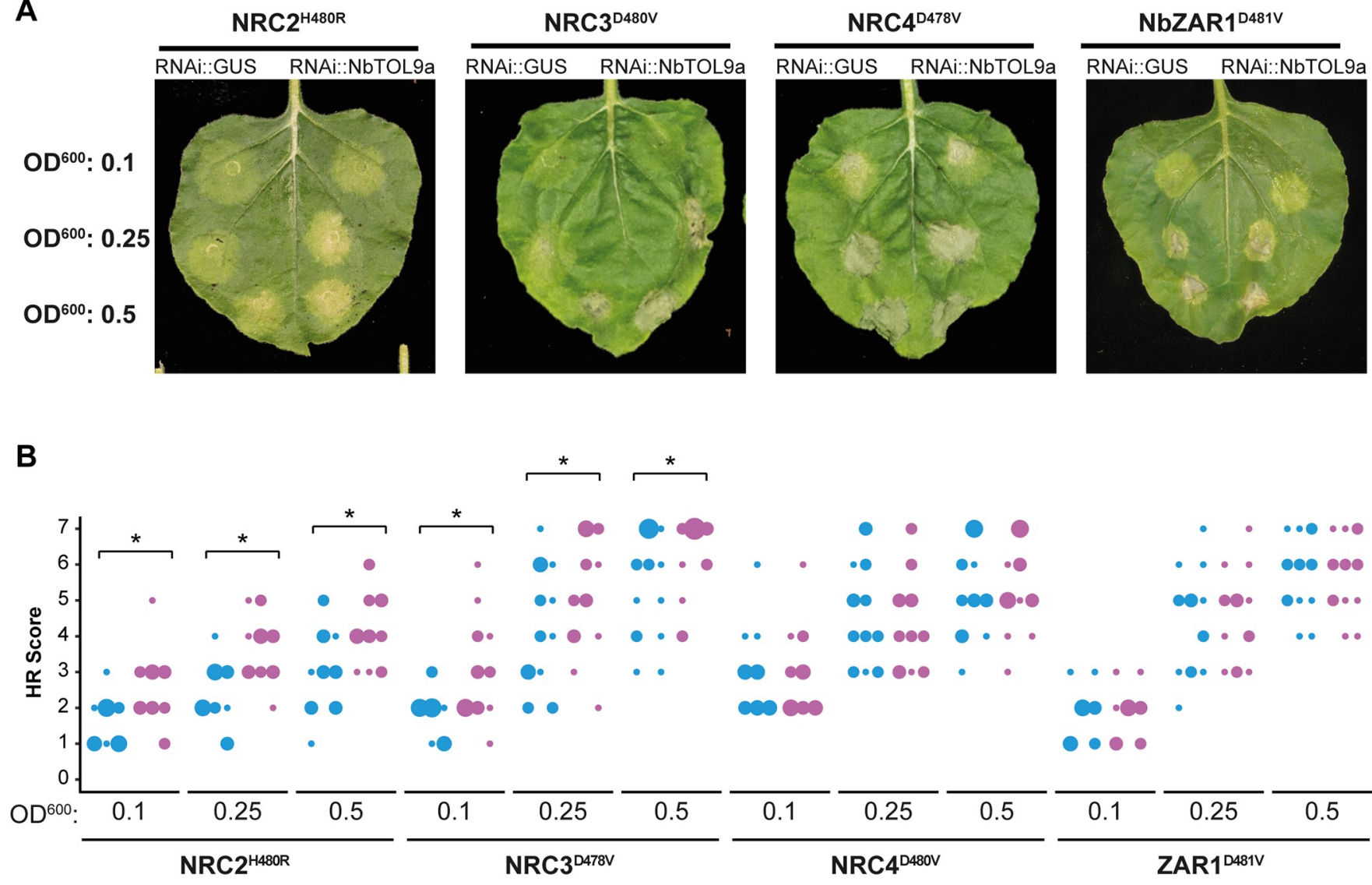

\section{Key RNAi::GUS}

RNAi::NbTOL9a

Count

- 2.5

5.0

7.5

10.0

Fig 10. Silencing of NbTOL9a enhances cell death mediated by NRC2 ${ }^{\mathrm{H} 480 \mathrm{R}}$ and NRC3 ${ }^{\mathrm{D} 480 \mathrm{~V}}$ but not NbZAR1 ${ }^{\mathrm{D} 481 \mathrm{~V}}$ or NRC4 ${ }^{\mathrm{D} 478 \mathrm{~V}}$. (A) Photo of representative $N$. benthamiana leaves showing HR after coexpression of $\mathrm{NRC} 2{ }^{\mathrm{H} 480 \mathrm{R}}, \mathrm{NRC} 3^{\mathrm{D} 480 \mathrm{~V}}, \mathrm{NRC} 4^{\mathrm{D} 478 \mathrm{~V}}$, and NbZAR $1^{\mathrm{D} 481 \mathrm{~V}}$ with RNAi::GUS (control) and RNAi:NbTOL9a (labelled above leaf panels). To improve the robustness of the assay, we used increasing concentrations of A. tumefaciens expressing $\mathrm{NRC}^{\mathrm{H} 480 \mathrm{R}}, \mathrm{NRC}^{\mathrm{D} 480 \mathrm{~V}}, \mathrm{NRC}^{\mathrm{D} 478 \mathrm{~V}}$, and NbZAR1 ${ }^{\mathrm{D} 481 \mathrm{~V}}\left(\mathrm{OD}_{600}=0.1,0.25\right.$, or 0.5$)(\mathrm{S} 10$ Data $)$. HR response was scored and photographed 5 days after agroinfiltration. (B) HR results are presented as dot plots, where the size of each dot is proportional to the number of samples with the same score (count). Three biological replicates were completed, indicated by columns for RNAi::GUS and RNAi::NbTOL9a, for each treatment combination. Statistical tests were implemented using the besthr R library [41]. We performed bootstrap resampling tests using a lower significance cutoff of 0.025 and an upper cutoff of 0.975 . Mean ranks of test samples falling outside of these cutoffs in the control samples bootstrap population were considered significant. Significant differences between the conditions are indicated with an asterisk (*). The details of statistical analysis are presented in (S14 Fig). HR, hypersensitive response.

https://doi.org/10.1371/journal.pbio.3001136.g010

affect NRC4 ${ }^{\text {D478V }}$ or NbZAR1 ${ }^{\text {D481V }}$ (NRC-independent NLR; [54]), compared to the RNAi:: GUS silencing control (Figs 10 and S14 and S10 Data).

Next, we determined the effect of NbTOL9a overexpression by coexpressing it with the autoimmune NRCs in N. benthamiana leaves using agroinfiltration. Since $\mathrm{NRC} 2^{\mathrm{H} 480 \mathrm{R}}$ autoimmune response is comparably weaker than $\mathrm{NRC}^{\mathrm{D} 480 \mathrm{~V}}$, we focused on $\mathrm{NRC}^{\mathrm{D} 480 \mathrm{~V}}$ in this and subsequent experiments as it provides a more robust readout for cell death-based assays. NbTOL9a overexpression reduced the cell death response triggered by NRC3 ${ }^{\mathrm{D} 480 \mathrm{~V}}$ but did not affect NRC4 ${ }^{\mathrm{D} 478 \mathrm{~V}}$ or the constitutively active $\mathrm{MEK} 2^{\mathrm{DD}}$, a mitogen-activated protein kinase kinase (MAPKK) involved in plant immune signalling, which we included as an additional control (Figs 11 and S15 and S11 Data). Altogether, these 2 sets of experiments indicate that NbTOL9a modulates NRC2 and NRC3 activities in a manner consistent with a negative regulatory role in NRC2- and NRC3-mediated immunity. 
A

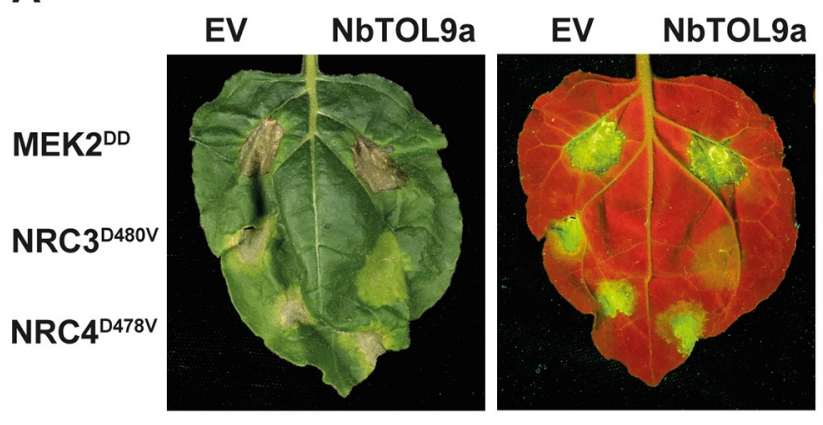

B
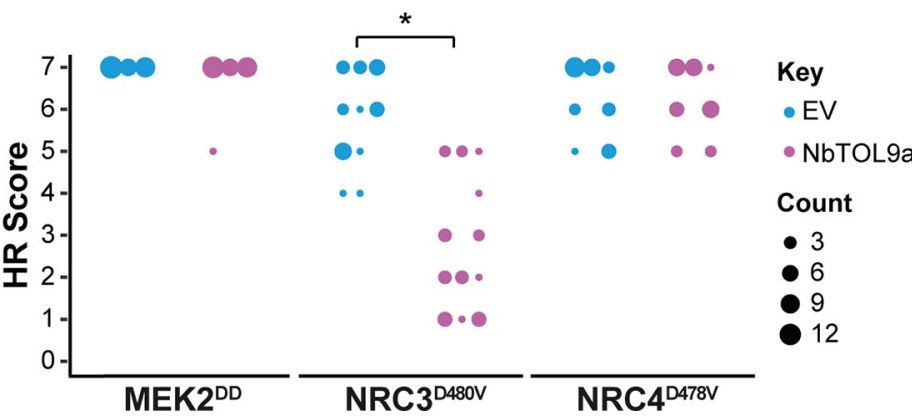

Fig 11. Overexpression of NbTOL9a suppresses autoactive $\mathrm{NRC}^{\mathrm{D} 480 \mathrm{~V}}$ but not MEK2 ${ }^{\mathrm{DD}}$ or NRC4 ${ }^{\mathrm{D} 478 \mathrm{~V}}$. (A) Photo of representative $N$. benthamiana leaves showing HR after coexpression of EV and NbTOL9a (labelled above leaf panels) with MEK2 ${ }^{\mathrm{DD}}$, NRC ${ }^{\mathrm{D} 480 \mathrm{~V}}$, and NRC4 ${ }^{\mathrm{D} 478 \mathrm{~V}}$. HR response was scored and photographed 5 days after agroinfiltration (left panel under white light, right panel autofluorescence under UV light). MEK $2^{\mathrm{DD}}$ was included as a positive control for cell death (S11 Data). (B) HR results are presented as dot plots, where the size of each dot is proportional to the number of samples with the same score (count). Three biological replicates were completed, indicated by columns for EV, NbTOL9a in each treatment $\left(\mathrm{MEK} 2^{\mathrm{DD}}, \mathrm{NRC} 3^{\mathrm{D} 480 \mathrm{~V}}, \mathrm{NRC} 4^{\mathrm{D} 478 \mathrm{~V}}\right)$. Statistical tests were implemented using the besthr R library [41]. We performed bootstrap resampling tests using a lower significance cutoff of 0.025 and an upper cutoff of 0.975 . Mean ranks of test samples falling outside of these cutoffs in the control samples bootstrap population were considered significant. Significant differences between the conditions are indicated with an asterisk (*). Details of statistical analysis are presented in (S15 Fig). EV, empty vector; HR, hypersensitive response.

https://doi.org/10.1371/journal.pbio.3001136.g011

\section{AVRcaplb suppression of NRC3 is compromised in the absence of NbTOL9a}

Our finding that NbTOL9a exhibits a negative regulatory role in NRC2- and NRC3-mediated cell death led us to the hypothesis that AVRcaplb is co-opting NbTOL9a to execute its suppression activity. To test this, we coexpressed AVRcaplb with the autoimmune mutants $\mathrm{NRC} 3^{\mathrm{D} 480 \mathrm{~V}}$ or NRC4 ${ }^{\mathrm{D} 478 \mathrm{~V}}$ in N. benthamiana leaves that are expressing either RNAi:: NbTOL9a (NbTOL9a silenced) or RNAi::GUS (negative control). Consistent with Fig 2, overexpression of AVRcap $1 \mathrm{~b}$ suppressed the cell death triggered by NRC $3^{\mathrm{D} 480 \mathrm{~V}}$ but not by $\mathrm{NRC}^{\mathrm{D} 478 \mathrm{~V}}$ (Fig 12, S12 Data). However, silencing of NbTOL9a compromised AVRcap1b suppression of $\mathrm{NRC}^{\mathrm{D} 480 \mathrm{~V}}$ autoimmunity and partially restored the cell death phenotype (Fig 12, S12 Data). These results indicate that AVRcap1b genetically requires NbTOL9a to fully downregulate NRC3 cell death activity and is likely co-opting this host protein to execute its suppression activities.

\section{NbTOL9a does not associate with NRCs in planta}

Given that NbTOL9a negatively regulates NRC2 and NRC3, we hypothesised that NbTOL9a associates with NRC2 and NRC3 to execute its immunomodulatory functions. Furthermore, since AVRcap1b requires NbTOL9a to fully suppress NRC3, AVRcap1b may act as a suppressor through altering NbTOL9a-NRC associations. To test these hypotheses, we coexpressed NbTOL9a::6xHA with NRC2::4xMyc, NRC3::4xMyc, and NRC4::4xMyc, either in the presence of GFP::AVRcap1b or free GFP in N. benthamiana leaves using agroinfiltration, and performed anti-Myc immunoprecipitation followed by western blot analyses. We included AVRcap 1b::4xMyc as a positive control for association with NbTOL9a. While we were able to successfully detect association between NbTOL9a:6xHA and AVRcap1b::4xMyc, we did not observe any association between NbTOL9a:6xHA and any of the 3 NRC proteins tested, regardless of whether GFP::AVRcap1b was present or absent (Fig 13). These results suggest that NbTOL9a-mediated negative regulation of NRC2 and NRC3 does not involve association between these 2 proteins in planta or, alternatively, involves protein-protein interactions that 
A

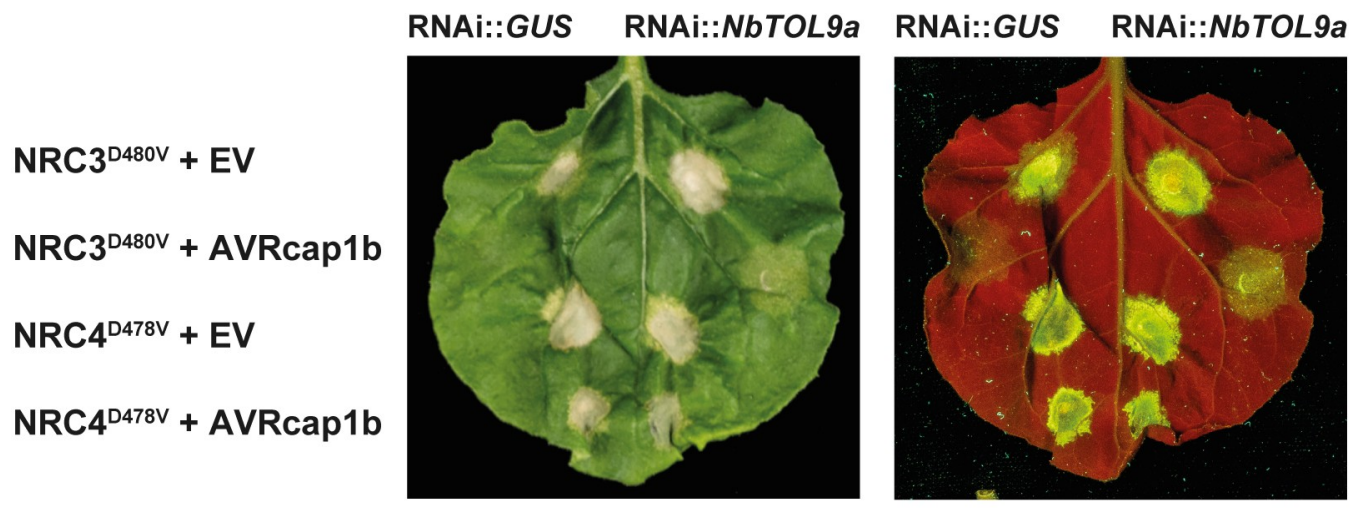

B

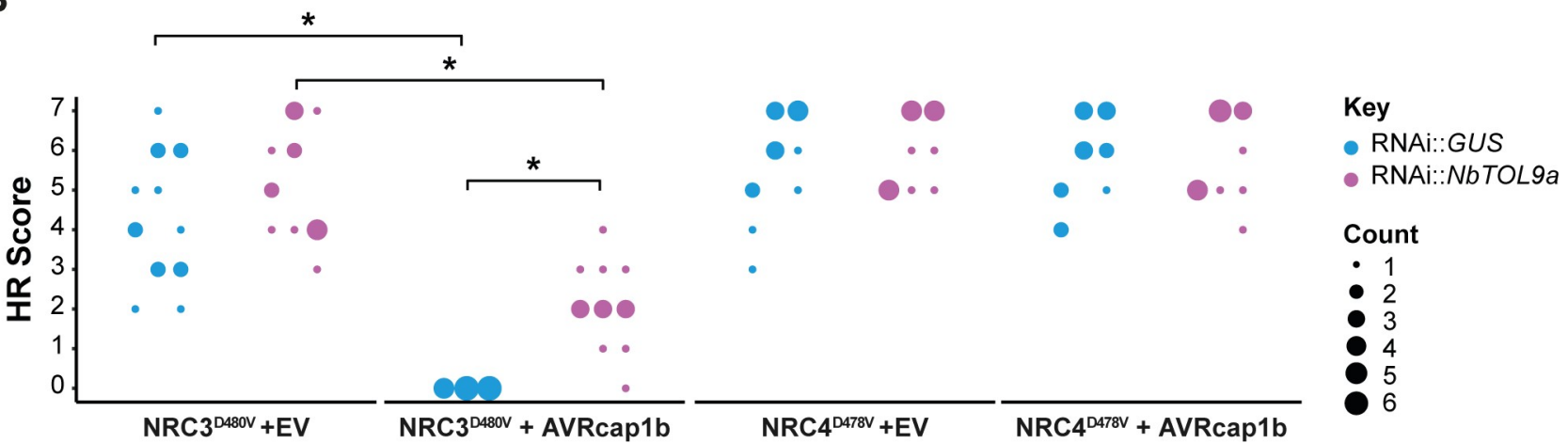

Fig 12. Silencing of NbTOL9a compromises AVRcap1b-mediated suppression of NRC3. (A) Photo of representative N. benthamiana leaves showing HR after coexpression of RNAi::GUS and RNAi::NbTOL9a with NRC $3^{\text {D480V }}+\mathrm{EV}, \mathrm{NRC} 3^{\mathrm{D} 480 \mathrm{~V}}+$ AVRcap1b, NRC4 $4^{\text {D478V }}+\mathrm{EV}$, and NRC4 ${ }^{\text {D478V }}$

+ AVRcap1b. HR response was scored and photographed 5 days after agroinfiltration (left panel under white light, right panel autofluorescence under UV light) (S12 Data). (B) HR results are presented as dot plots, where the size of each dot is proportional to the number of samples with the same score (count). Results are based on 3 biological replicates. Statistical tests were implemented using the besthr R library [41]. We performed bootstrap resampling tests using a lower significance cutoff of 0.025 and an upper cutoff of 0.975 . Mean ranks of test samples falling outside of these cutoffs in the control samples bootstrap population were considered significant. Significant differences between the conditions are indicated with an asterisk $\left({ }^{*}\right)$. Details of statistical analysis are presented in (S16 Fig). HR, hypersensitive response.

https://doi.org/10.1371/journal.pbio.3001136.g012

are too transient to be detected by coIP. Moreover, we conclude that AVRcap1b does not alter NbTOL9a-NRC associations (or lack thereof) to execute its immune suppression activities.

\section{Discussion}

The aim of this study was to address the hypothesis that solanaceous parasites have evolved effector proteins that target the NRC network of NLR immune receptors. We confirmed this hypothesis by carrying out an effectoromics screen, which yielded 5 effectors that can compromise the NRC network: SS10, SS15, and SS34 from the cyst nematode G. rostochiensis and AVRcap1b and PITG-15278 from the potato late blight pathogen P. infestans. These 5 effectors can suppress the hypersensitive cell death induced in N. benthamiana by either Prf or Rpiblb2, 2 NRC-dependent sensor NLRs that function as bona fide disease resistance proteins. Interestingly, these effectors appear to function at different points in the NRC network (Fig 14). While SS10, SS34, and PITG- 15278 suppress cell death mediated by Rpi-blb2, they do not interfere with an autoimmune mutant of the downstream helper NRC4. SS15 and AVRcap1b, however, can robustly suppress autoimmune mutants of NRC2 and NRC3, indicating that they act at the level of the NRC helpers or their downstream pathways. We found that SS15 directly binds the NB-ARC domain of NRC2 and NRC3, while AVRcap1b associates with NbTOL9a and requires this host protein to fully suppress NRC3. We conclude that cyst 


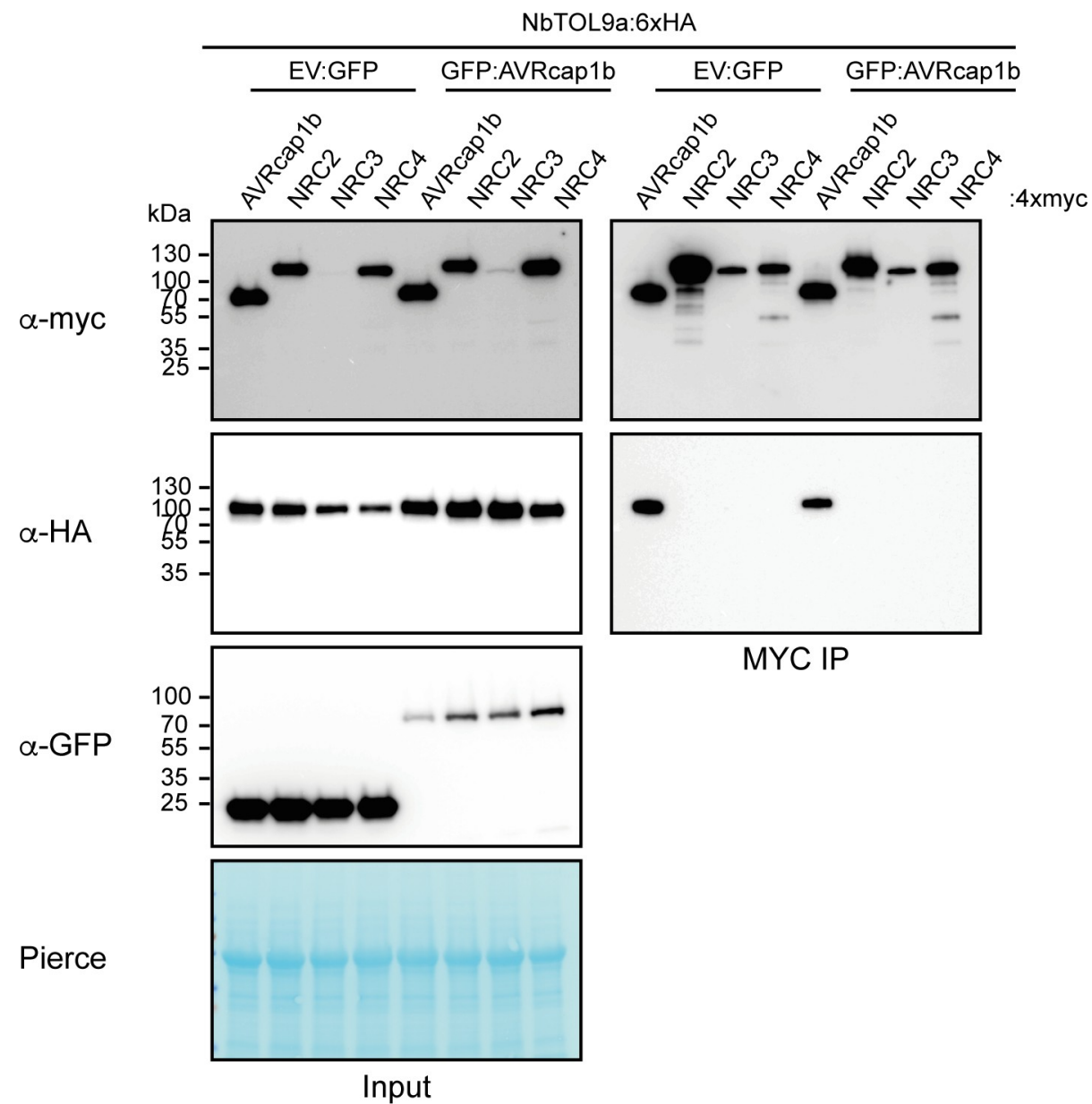

Fig 13. NbTOL9a does not associate with NRCs in planta. CoIP experiment between NbTOL9a and NRCs in the presence and absence of AVRcap1b. C-terminally 6xHA-tagged NbTOL9a was transiently coexpressed with NRC2, NRC3, and NRC4 proteins fused to a C-terminal 4xmyc tag in the presence of free GFP (EV:GFP) or N-terminally GFP-tagged AVRcap1b. C-terminally 4xmyc-tagged AVRcap1b was used as a positive control for association with NbTOL9a. IP was performed with agarose beads conjugated to MYC (MYC-IP) antibodies. Total protein extracts were immunoblotted with appropriate antisera labelled on the left. Approximate molecular weights $(\mathrm{kDa})$ of the proteins are shown on the left. Rubisco loading controls were conducted using Pierce staining. This experiment is representative of 2 independent replicates. coIP, co-immunoprecipitation; IP, immunoprecipitation; NRC, NLR required for cell death.

https://doi.org/10.1371/journal.pbio.3001136.g013

nematodes and $P$. infestans convergently evolved sequence unrelated effectors that target key nodes of the NRC network or their downstream components to suppress host immune signalling. Our paper also highlights the value of using effectors as probes to dissect key regulatory components of immunity and to study the complex interactions between NLR receptors and the networks they form.

Our findings help to explain why plants have evolved NLR receptor networks with complex architectures. We previously postulated that NLR networks, such as the NRC network, help maintain the robustness of the immune system in light of external perturbations [55]. A key feature of the NRC network is that NRCs act as central nodes that function downstream of multiple disease resistance proteins (NLR sensors) that form a massively expanded phylogenetic clade in the Solanaceae. The NRC nodes have overlapping NLR sensor specificities and display varying degrees of redundancy [24]. Given that NRCs are critical for immune signalling of multiple disease resistance proteins, they would be ideal targets for pathogen effectors. 


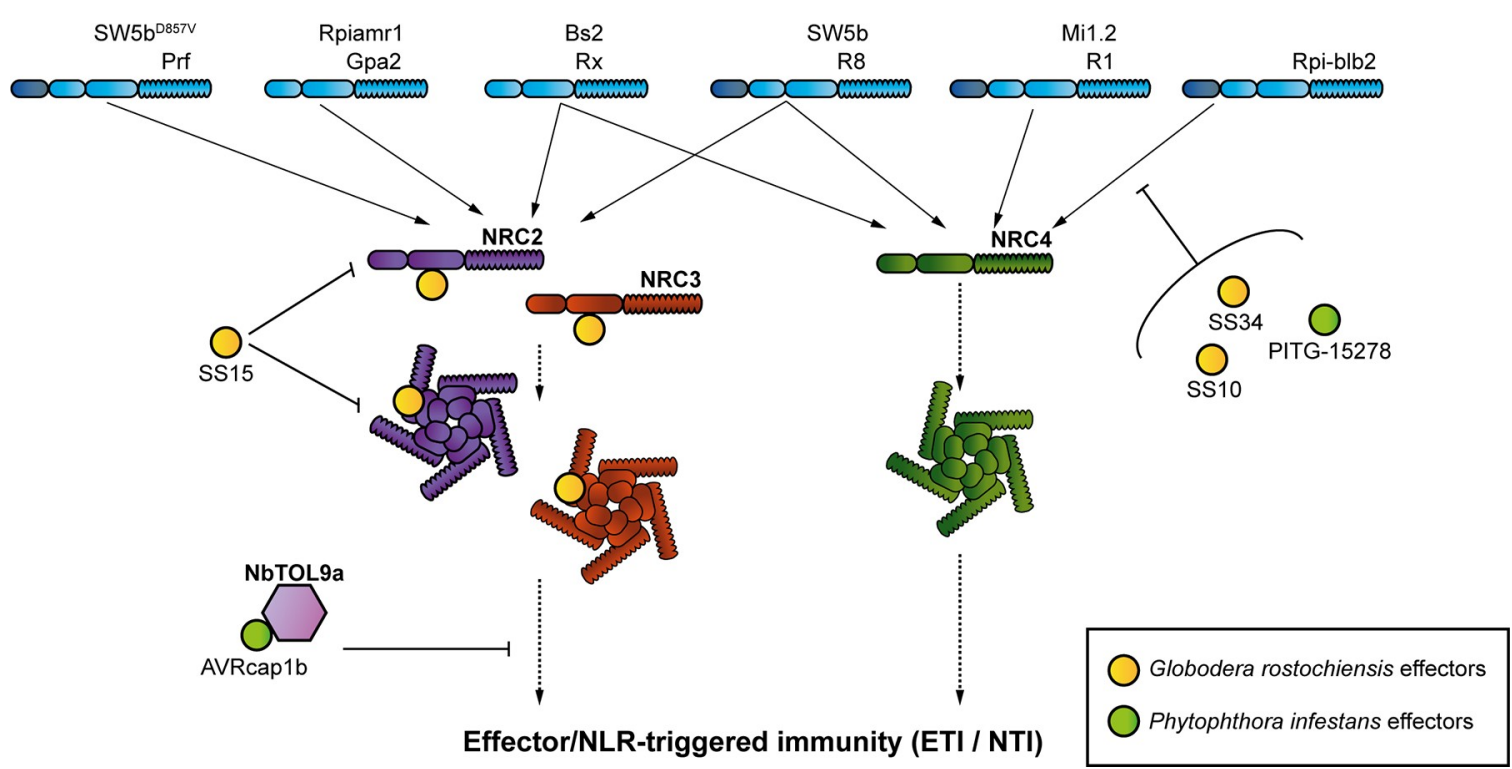

Fig 14. Evolutionary divergent pathogens have evolved to target multiple layers of the Solanaceae NLR network. P. infestans and G. rostochiensis, 2 evolutionary distinct pathogens, have evolved effectors that suppress signalling mediated by the NRC network. Two effectors from the cyst nematode pathogen, G. rostochiensis (SS10 and SS34), and 1 effector from the blight pathogen, P. infestans (PITG15278) suppress the function of the NRC4-dependent sensor NLR, Rpi-blb2. The cyst nematode effector, SS15, binds to the NB-ARC domain of both inactive and activated forms of NRC2 and NRC3. By binding the NB-ARC domain of NRC2 and NRC3, SS15 is able to suppress their function. The P. infestans effector, AVRcap1b, suppresses the function of NRC2 and NRC3, and in the case of NRC3, AVRcap1b suppression requires the ESCRT-related protein NbTOL9a. ESCRT, endosomal sorting complex required for transport; ETI/ NTI, effector/NLR-triggered immunity; NB-ARC, nucleotide-binding domain shared with APAF-1, various R-proteins, and CED-4; NLR, nucleotide-binding domain and leucine-rich repeat; NRC, NLR required for cell death.

https://doi.org/10.1371/journal.pbio.3001136.g014

Our finding that 2 distantly related pathogens, an oomycete and a cyst nematode, evolved effectors to suppress NRC signalling through distinct mechanisms, supports this hypothesis. It is tempting to speculate that NRC redundancy has, therefore, emerged as a strategy to enhance the plant's capacity to evade immune suppression. For example, although both SS15 and AVRcap $1 \mathrm{~b}$ are robust suppressors of NRC2 and NRC3, they are not able to suppress their paralog NRC4. This redundancy would allow the host to mount an effective immune response even in the presence of NRC2 and NRC3 suppressors. However, it should be noted that while we did observe binding between $\mathrm{SS} 15$ and NRC4 in $\mathrm{Y} 2 \mathrm{H}$ screens, their association was very weak or not detectable in planta. It is possible that physiological conditions in the plant do not favour association between SS15 and NRC4, which is consistent with our observations that SS15 cannot suppress NRC4 signalling. Interestingly, the majority of NRC-dependent potato blight R genes, e.g., R1, R8, and Rpi-blb2, signal through NRC4 [24]. NRC4 may therefore have evolved as the helper NLR that predominantly functions with late blight $\mathrm{R}$ genes because it evades suppression by $P$. infestans. However, Rpi-amr1 was recently shown to require NRC2 and NRC3 for resistance to $P$. infestans, indicating that the interactions between sensor NLRs, helper NRCs, and pathogen effectors are extremely complex [56]. Nonetheless, this work supports the hypothesis that NLR networks make plant immune systems more resilient through redundant signalling architectures. The extent to which pathogen effectors target NLR networks and the molecular mechanisms by which they do so, however, are still not fully understood.

An emerging paradigm in plant immunity is that NTI and PTI signalling employ common modules and reinforce each other, blurring the division between these 2 classes of plant immunity [57-60]. It is possible that NTI suppressing effectors, such as AVRcap1b and SS15, simultaneously compromise both NTI and PTI by targeting shared immune signalling nodes. 
Therefore, identification of NTI suppressors may translate into new insights regarding basal resistance and help us further decipher the intricate nature of the plant immune system.

While the precise molecular mechanism that the cyst nematode effector SS15 utilises to suppress NRC2 and NRC3 remains to be determined, our experiments provide some important insights. SS15 binds both inactive (Figs 6B and S9 and S10) and constitutively active forms of NRCs (Fig 8), presumably through their NB-ARC domain (Fig 7). There are only a few examples of effectors that directly bind NLRs to suppress their activities. NleA, an effector from human enteropathogenic E. coli was shown to suppress the NOD-like receptor (NLR) NLRP3 by directly binding to it, thereby interfering with deubiquitination, which is critical for inflammasome activation [61]. NleA associates with both ubiquitinated and nonubiquitinated NLRP3 by interacting with the PYD and LRR domains [62]. While the exact mechanism utilised by NleA is currently unknown, the authors theorise that binding of NleA to the PYD and LRR domains may be responsible for inhibition of NLRP3 inflammasome formation by preventing NLRP3 interaction with other downstream signalling partners or blocking access of the deubiquitinating enzyme to the polyubiquitinated NLR [62]. P. infestans IPI-O4 is a plant pathogen effector reported to bind NLRs to suppress host immunity. Chen and colleagues [63] and Karki and colleagues [64] showed that the P. infestans effector IPI-O4 compromises the HR mediated by the NLR disease resistance protein RB (also known as Rpi-blb1) by directly binding to its $\mathrm{N}$-terminal CC domain, possibly to compete with binding by the AVR effector AVRblb1, a homolog of IPI-O4. Therefore, IPI-O4 acts directly on the sensor NLR and is more reminiscent of the 3 Rpi-blb2 suppressors we report here than to the NRC suppressors AVRcaplb and SS15 (Fig 13).

The recent elucidation of the ZAR1, RPP1, and ROQ1 structures have revealed that structural remodelling of the NB-ARC domain, a region involved in NLR activation, is essential for resistosome formation [27-30]. The mechanism utilised by SS15 to suppress NRC-mediated immunity may involve tampering with NB-ARC structural rearrangements. In mammalian systems, for example, a compound known as MCC950 directly binds both the inactive and activated forms of NLRP3 to inhibit its function $[65,66]$. MCC950 binds the central NACHT domain, the mammalian equivalent of the NB-ARC domain of plant NLRs, to interfere with ATP hydrolysis and prevent conformational changes that are critical for NLRP3 activation and subsequent inflammasome assembly. This ultimately drives NLRP3 towards a closed and inactive conformation $[65,66]$. Based on our findings, we propose that SS15 could be acting as an NLR inhibitor that directly perturbs NRC activities by binding the NB-ARC domain and forcing NRC2 and NRC3 into an inactivated state, possibly through mechanisms analogous to MCC950. Further studies investigating the extent to which SS15 perturbs structural remodelling of the NB-ARC domain of NRCs will provide mechanistic insights into how this effector is able to suppress NRC2 and NRC3.

Unlike SS15, AVRcap1b from the potato late blight pathogen P. infestans indirectly suppresses the function of autoimmune NRC2 and NRC3 and therefore likely targets host proteins downstream of these cell death executor NLRs. We identified NbTOL9a, a member of the TOL protein family, as a host target of AVRcaplb and showed that this host protein acts as a negative modulator of NRC3-mediated hypersensitive cell death. TOL proteins are key components of the ESCRT machinery and have well characterised roles in intracellular protein trafficking $[52,67,68]$. They act as ubiquitin receptors in the early steps of the ESCRT trafficking pathway by interacting with ubiquitinated cargo via their ENTH/VHS and GAT domains [51-53]. Conlan and colleagues [69] identified a TOL family member that is proximal to the $P$. syringae effector AvrPto, when this effector protein was transiently expressed in N. benthamiana leaf tissue. In addition, silencing of this TOL protein resulted in decreased growth of $P$. syringae pv. tabaci on N. benthamiana, which is in line with our observation that TOL proteins 
can act as negative regulators of plant immune signalling. The fact that AvrPto is proximal to TOLs further links the NRC network to TOL proteins, since AvrPto is recognised by the NRC2- and NRC3-dependent sensor NLR Prf. Together, these findings strengthen our hypothesis that TOLs can act as negative regulators of this complex immune signalling network. The precise mechanism TOLs utilise to modulate plant immunity, however, is still unknown. In the case of NbTOL9a, this negative regulation does not seem to involve proteinprotein interactions between NbTOL9a and NRCs. In mammalian systems, the ESCRT pathway is involved in negatively regulating several forms of programmed cell death, including necroptosis and pyroptosis, by repairing damaged sections of the plasma membrane [70-72]. It is possible that AVRcap1b is co-opting NbTOL9a to hijack a similar immunomodulatory trafficking pathway in the plant cell to counteract NRC-mediated HR cell death and suppress immunity. This model would be consistent with the observation that vesicle trafficking is massively reprogrammed by $P$. infestans effectors during infection [49,73-75].

Interestingly, even though AVRcap1b and SS15 are robust NTI suppressors, they can activate immunity on certain Solanaceae species. AVRcaplb, for example, can activate immunity on accessions of Solanum capsicibaccatum carrying the NLR disease resistance gene Rpi-cap1hence the moniker AVR $[32,76]$. SS15, on the other hand, is recognised by a yet to be described protein in Nicotiana tabacum resulting in HR cell death [35]. The fact that both AVRcap1b and SS15 act as both triggers and suppressors of NLR immunity further highlights the complex coevolutionary dynamics that exist between effectors and NLRs/NLR networks and the need for studies that take into account these intricate epistatic interactions. Additionally, understanding the interplay between AVRcap1b, TOLs, and NRCs will help advance our knowledge of the regulatory mechanisms that govern plant immunity and determine the outcome of multipartite host-pathogen interactions mediated by a complex immune receptor network.

The field of NLR biology has seen significant progress over the past few years, and yet the genetic components and immune signalling pathways downstream of NLR activation remain obscure [77]. Moreover, the precise molecular mechanisms that underpin the activation of paired and networked NLRs and subsequent cell death response are still unknown. Here, we have gained insights into the molecular strategies that plant pathogens utilise to counteract host immune function. We identified 5 effectors (SS15, AVRcap1b, PITG-15278, SS10, and SS34) that compromise components of the NRC network. We focused on SS15 and AVRcap1b, as these effectors counteract NRCs-MADA-type CC-NLRs-which form central nodes within the immune receptor network. SS15 and AVRcap1b, therefore, have the unique potential to uncover valuable mechanistic details regarding the activation of MADA-type CC-NLR resistosomes and their downstream signalling elements. The fact that these distantly related pathogens, an oomycete and a cyst nematode, have independently evolved effectors to counteract NRC2 and NRC3 further highlights the critical role NRCs play in mediating immunity to solanaceous parasites. Beyond SS15 and AVRcap1, there is still much we can learn by studying PTIG-15278, SS10, and SS34, the 3 Rpi-blb2 suppressors identified in this study. Further research may allow us to determine the target(s) and mechanism(s) utilised by these 3 effectors to suppress host immunity. Recently, effectors from the oomycete pathogen Phytophthora capsici and the aphid pest $M$. persicae were shown to converge on host E3 SUMO ligase SIZ1 to suppress plant immunity [78]. Taken together with our work, this suggests that microbial pathogens, herbivorous insects, and parasitic nematodes may share more common virulence mechanisms than anticipated. Studying immunosuppressors holds the potential to advance our understanding of the functional principles and evolutionary dynamics that underpin plant immune receptor networks. This knowledge can then be leveraged to guide new approaches for breeding disease resistance to maximise crop protection, for example, by engineering NLRs that evade pathogen suppression. 


\section{Materials and methods}

\section{Plant growth conditions}

Wild-type and Rx-transgenic [79] $N$ benthamiana lines were grown in a controlled growth chamber with temperature 22 to $25^{\circ} \mathrm{C}$, humidity $45 \%$ to $65 \%$, and 16/8-hour light/dark cycle.

\section{Gene cloning and synthesis}

Effector library. All sequence information for pathogen effectors used in suppression assays can be found in S1 Table.

P. infestans effectors, without signal peptide [33,34], were codon optimised for Solanum tuberosum and synthesised by GENEWIZ (South Plainfield, NJ, United States of America) into pUC57-Amp. Effector sequences were amplified from the pUC57-Amp vector by Phusion High-Fidelity DNA Polymerase (Thermo Fisher Scientific, MA, USA), and the purified amplicon was used directly for Golden Gate assembly into the Level 0 Universal Acceptor pUAP1 (The Sainsbury Laboratory (TSL) SynBio, addgene no. 63674). Primers used for PCR amplification are listed in S6 Table. Expression constructs were generated by Golden Gate assembly of the Level 0 module into binary vector pICSL86977OD [with cauliflower mosaic virus (CaMV) $35 \mathrm{~S}$ promoter and octopine synthase gene) terminator] (addgene no. 86180). PITG05910 and PITG-22926 were synthesised by GENEWIZ (South Plainfield, NJ, USA) into pUC57-kan as Golden Gate L0 modules, then subcloned into binary vector pICH47742 [addgene no. 48001], together with $\mathrm{pICH} 51266$ [35S promoter $+\Omega$ promoter, addgene no. 50267], and pICH41432 [octopine synthase terminator, addgene no. 50343]. All constructs were verified by sequencing.

G. rostochiensis effectors SS4, SS8, SS9, SS15, SS16, and SS19 cloned into pBINPLUS expression vector with $\mathrm{N}$-terminal 4xHA tag were provided by Aska Goverse (Laboratory of Nematology, Wageningen University, the Netherlands) $[36,80]$. G. pallida effector sequences $12 \mathrm{~N} 3$, $33 \mathrm{H} 17$ [81] were synthesised by GENEWIZ (South Plainfield, NJ, USA) as Golden Gate Level 0 modules into pICH41155, Gpa-SS37 [82] was synthesised by GENEWIZ (South Plainfield, NJ, USA) as a Golden Gate Level 0 module into pUC57-kan. The remaining G. rostochiensis effector sequences were extracted from the https://parasite.wormbase.org website [37] and synthesised by GENEWIZ (South Plainfield, NJ, USA) as Golden Gate Level 0 modules into pUC57-kan. These effectors were subcloned into binary vector pICH47732 [addgene no. 50434], together with pICH51266 [35S promoter $+\Omega$ promoter, addgene no. 50267], and pICH41432 [octopine synthase terminator, addgene no. 50343] for cell death suppression assays.

The green peach aphid (M. persicae) and pea aphid (A.pisum) effectors cloned into pCB302-3 in A. tumefaciens strain GV3101::pM90 were provided by Saskia Hogenhout (John Innes Centre, Norwich, United Kingdom). The tomato bacterial speck pathogen (P. syringae) effectors cloned into pEarly Gate 100 in A. tumefaciens strain C58C1 were kindly provided by Wenbo Ma (previously University California, Riverside, USA, currently The Sainsbury Laboratory, Norwich, UK).

Synthesis of NRC constructs. Full-length $N$. benthamiana $\mathrm{NRC}^{2} \mathrm{a}^{\mathrm{syn}}$ (NCBI accession number KT936525) [24], NRC3 ${ }^{\mathrm{WT}}$, and $\mathrm{NRC} 4^{\mathrm{WT}}$ were synthesised by GENEWIZ (South Plainfield, NJ, USA) into pICH41155 as Golden Gate L0 modules (S7 Table). These sequences were manually domesticated to remove BpiI and BsaI sites.

Site-directed mutagenesis of NRC2, NRC3, and SW5b. Autoactive mutants of NRC2, NRC3, and SW5b were generated by introducing a histidine $(\mathrm{H})$ to arginine $(\mathrm{R})$ mutation in the MHD motif of NRC2 and an aspartic acid (D) to valine (V) substitution in the MHD motif 
of NRC3 and SW5b independently. Primers listed in S8 Table were used for introducing mutations by inverse PCR with Phusion High-Fidelity DNA Polymerase (Thermo Fisher Scientific). pICH41155::NRC2, PCR8::NRC3 ${ }^{\text {WT }}$ [26], and PICH441155::SW5b [24] were used as templates for site-directed mutagenesis of NRC2, NRC3, and SW5b, respectively. The mutated variants were verified by sequencing and then subcloned into pICSL86977OD [addgene no. 86180] (for $\mathrm{NRC} 2^{\mathrm{H} 480 \mathrm{R}}$ and $\mathrm{SW} 5 \mathrm{~b}^{\mathrm{D} 847 \mathrm{~V}}$ ) and pICH86988 [addgene no. 48076] $\left(\mathrm{NRC}^{\mathrm{D} 480 \mathrm{~V}}\right.$ ). Autoactive NRC4 mutants used in this study were described previously [25]. Verified plasmids were then transformed into GV3101:pM90 for cell death assay screens.

To determine whether an intact p-loop is essential for SS15 in planta association to NRC2 and NRC3, a lysine $(\mathrm{K})$ to arginine $(\mathrm{R})$ mutation was introduced independently into the $\mathrm{p}$ loops of both proteins by site-directed mutagenesis using Phusion High-Fidelity DNA Polymerase (Thermo Fisher Scientific). pCR8::NRC2 ${ }^{\mathrm{WT}}$-ns and PCR8::NRC3 ${ }^{\mathrm{WT}}$-ns [26] were used as a templates. Primers used to for introducing mutations are listed in S8 Table. The mutated NRC variants were verified by sequencing and subcloned into pICH86988 [addgene no. 48076] together with pICSL50010 [C-terminal 4xMyc tag, addgene no. 50310]. Both constructs were verified by sequencing and transformed into A. tumefaciens GV3101::pM90.

Cloning into $E$. coli expression vectors for protein purification. SS15: DNA encoding SS15 residues Ser-25 to Ile-246 (lacking signal peptide) was amplified from pBINPLUS::4HA:: SS15 plasmid (using primers shown in S15 Table) and cloned into the pOPINS3C vector resulting in an $\mathrm{N}$-terminal 6xHis-SUMO tag with SS15, linked by a $3 \mathrm{C}$ cleavage site (pOPINS3C:SS15) [83].

NB-ARC domains of NRC2, NRC3, and NRC4: For NRC2, 2 NB-ARC domain constructs with alternative domain boundaries were generated. DNA encoding NRC2 NB-ARC domain residues Val-148 to Tyr-508 (NRC2 ${ }^{148-508}$ ) or Val-148 to Asn-496 (NRC2 ${ }^{148-496}$ ) was amplified from pICH41155::NRC2 $\mathrm{a}^{\text {syn }}$ (using primers shown in $\mathrm{S} 15$ Table) and cloned into the vector pOPINS3C resulting in an N-terminal 6xHis-SUMO tag with NRC2 NB-ARC, linked by a 3C cleavage site (pOPINS3C:NRC2 ${ }^{148-508}$ and pOPINS3C:NRC2 ${ }^{148-496}$, respectively). Similarly, DNA encoding NRC3 NB-ARC residues Val-152 to Ser-507 (NRC3 ${ }^{\mathrm{NB}-\mathrm{ARC}}$ ) was amplified from pICH41155::NRC $3^{\mathrm{WT}}$ (using primers shown in S15 Table) and DNA encoding NRC4 NB-ARC domain Ala-150 to Lys-491 (NRC4 ${ }^{\text {NB-ARC }}$ ) was amplified from pICH41155:: $\mathrm{NRC} 4{ }^{\mathrm{WT}}$ (using primers shown in S15 Table) and cloned into the vector pOPINS3C as described above (pOPINS3C:NRC3 ${ }^{\mathrm{NB}-\mathrm{ARC}}$ and pOPINS3C:NRC4 ${ }^{\mathrm{NB}-\mathrm{ARC}}$, respectively).

Generating C-terminally 4xMyc tagged NRC2 and NRC3 autoactive mutants. To generate $\mathrm{NRC} 2^{\mathrm{H} 480 \mathrm{R}}:: 4 \mathrm{xMyc}$ and $\mathrm{NRC} 3^{\mathrm{D} 480 \mathrm{~V}}:: 4 \mathrm{xMyc}$, the stop codon was removed from pICSL86977OD::NRC2 ${ }^{\mathrm{H} 480 \mathrm{R}}$ and pICH86988::NRC ${ }^{\mathrm{D} 480 \mathrm{~V}}$ by Phusion High-Fidelity DNA polymerase (Thermo Fischer Scientific) using primers in S8 Table. The purified amplicon was used directly for Golden Gate assembly into binary vector pICH86988 [addgene no. 48076], together with pICSL50010 [C-terminal 4xMyc tag, addgene no. 50310]. Both constructs were verified by DNA sequencing and then transformed into A. bacterium strain GV3101::pM90.

Generating N-terminally GFP-tagged, C-terminally 6xHA-tagged, and C-terminally 4xmyc-tagged AVRcaplb. AVRcaplb constructs used in coIP experiments were generated by Golden Gate assembly. To generate GFP::AVRcap1b, level 0 pUAP1::AVRcap1b was assembled into binary vector pICH86966 [addgene no. 48075] driven by pICSL13008 [CaMV 35S promoter $+5^{\prime}$ untranslated leader tobacco mosaic virus, TSL SynBio], pICSL30006 [GFP, addgene no. 50303], and pICH41432 [octopine synthase terminator, addgene no. 50343]. To generate AVRcap1b::6xHA and AVRcap1b:L4xmyc, the stop codon was removed from pUAP1::AVRcap1b by Phusion High-Fidelity DNA Polymerase (Thermo Fisher Scientific) using primers listed in $\mathrm{S} 6 \mathrm{Table}$. The purified amplicon was used directly for Golden Gate assembly into binary vector pICH47742 [addgene no. 48001] driven by pICH85281 
[mannopine synthase promoter $+\Omega$ (Mas $\Omega$ pro), addgene no. 50272], pICSL50009 [6xHA, addgene no. 50309], and pICSL60008 [Arabidopsis heat shock protein terminator (HSPter), TSL SynBio]. All constructs were verified by DNA sequencing and then transformed into $A$. bacterium strain GV3101::pM90.

Cloning of NbTOL paralogs. We used reciprocal BLAST searches of Nbv6.1trP4561, which was identified in both IP-MS and Y2H assays, and mined the V6.1 transcriptome (https://benthgenome.qut.edu.au/) for additional TOL sequences. We cross-validated the extracted TOLs with the genome-based predicted proteomes [84] and removed likely duplicates representing chimeric transcripts due to assembly of short read sequences. We identified a total of 5 TOL paralogs, which we termed NbTOL9a (Nbv6.1trP4361), NbTOL9b (Nbv6.1trP9166), NbTOL3 (Nbv6.1trA40123), NbTOL6 (Nbv6.1trP73492), and NbTOL9c (Nbv6.1trA64113) (S5 Table). NbTOL paralogs were synthesised using GENEWIZ (South Plainfield, NJ, USA) into pUC57-Kan as L0 modules. Individual TOL paralogs were subjected to PCR by Phusion High-Fidelity DNA Polymerase (Thermo Fisher Scientific) to remove the stop codon (primer information listed in S12 Table). The purified amplicons were used directly for Golden Gate assembly into binary vector pICH47732 [addgene no. 50434], together with $\mathrm{pICH} 51266$ [ $35 \mathrm{~S}$ promoter $+\Omega$ promoter, addgene no. 50267], pICSL50009 [6xHA, addgene no. 50309], and pICH41432 [octopine synthase terminator, addgene no. 50343]. All constructs were verified by DNA sequencing and transformed into A. tumefaciens GV3101::pM90.

\section{Cell death and suppression assays by agroinfiltration}

Information of constructs used for cell death assays are summarised in S9 Table. Transient expression of NLR immune receptors and cognate effectors and autoactive immune receptors with EV or effector constructs were performed according methods previously described [13]. Briefly, 4- to 5-week-old N. benthamiana plants were infiltrated with A. tumefaciens GV3101:: pM90 strains carrying the expression vectors of different indicated proteins within the text. $A$. tumefaciens suspensions were adjusted in infiltration buffer $(10 \mathrm{mM} \mathrm{MES}, 10 \mathrm{mM} \mathrm{MgCl}$, and $150 \mu \mathrm{M}$ acetosyringone ( $\mathrm{pH} 5.6)$ ) to final $\mathrm{OD}_{600}$ indicated in $\mathrm{S}$ Table. $\mathrm{OD}_{600}$ for all effectors were adjusted to 0.2. For NbTOL9a overexpression assays, NbTOL9a::6xHA construct was coinfiltrated at a final $\mathrm{OD}_{600}$ of 0.2 . The cell death, HR, phenotype was scored 5 to 7 days after agroinfiltration, unless otherwise stated, using a previously described scale [85], modified from 0 (no necrosis observed) to 7 (confluent necrosis).

\section{Virus-induced gene silencing (VIGS) of NRC homologs}

VIGS was performed in N. benthamiana as previously described [86]. Suspensions of A. tumefaciens strain GV3101::pM90 harbouring TRV RNA1 (pYL155) and TRV RNA2 (pYL279), with corresponding fragments from $N R C 2, N R C 3$, and $N R C 4$, were mixed in a 2:1 ratio in infiltration buffer (10 mM 2-[N-morpholine]-ethanesulfonic acid [MES]; $10 \mathrm{mM} \mathrm{MgCl}_{2}$; and $150 \mu \mathrm{M}$ acetosyringone ( $\mathrm{pH}$ 5.6)) to a final OD600 of 0.3. Two-week-old N. benthamiana plants were infiltrated with $A$. tumefaciens for VIGS assays; upper leaves were used 2 to 3 weeks later for further agroinfiltrations. The $N R C 2 / 3$ double silencing, $N R C 4$ and NRC2/3/4 triple silencing constructs were described previously [24,26].

\section{PVX infection assays (agroinfection)}

Plants were used 3 weeks after TRV infection. Plants were infected with PVX (pGR106) using the toothpick inoculation method, expressed via A.tumefaciens [24,46], and examined for the spread of trailing necrotic lesions from the inoculated spots [43]. pGR106::PVX::GFP 
construct generation was described previously [24]. One day before PVX toothpick inoculation, EV and pICH86977::AVRcap1b or pBINPLUS::4HA::SS15 constructs were expressed by agroinfiltration into leaves of $R x$ plants that were subjected to $N R C 2 / 3$ double, NRC4 and $N R C 2 / 3 / 4$ triple silencing. The infiltrated area was then circled with a marker pen. $N R C$ homologs were silenced by VIGS as described above in $R x$-transgenic $N$. benthamiana. Toothpicks were dipped into culture of $A$. tumefaciens harbouring the PVX-GFP vector and then used to pierce small holes in the leaves of $N$. benthamiana. Photos were taken at 3 weeks after PVX inoculation, and the size of the lesions were measured in Fiji (formerly ImageJ). Scatterplot of the lesion size was generated in $\mathrm{R}$ using the ggplot2 package, as described previously [87]. A core borer $\left(0.8 \mathrm{~cm}^{2}\right)$ was used to collect leaf discs from the inoculation sites 3 weeks after PVX inoculation for immunoblot with anti-GFP (B-2, sc9996 HRP, Santa Cruz Biotechnology, CA, USA). GFP accumulation was visualised with Pierce ECL Western (32106, Thermo Fisher Scientific) and where necessary up to 50\% SuperSignal West Femto Maximum Sensitivity Substrate (34095, Thermo Fisher Scientific).

\section{Phylogenetic analyses of Nicotiana benthamiana and Arabidopsis thaliana TOL proteins}

Amino acid sequences of the NbTOL paralogs identified in N. benthamiana and previously published A. thaliana AtTOL proteins [53,88] were aligned using Clustal Omega [89]. The alignment was then manually edited in MEGAX [90]. The gaps in the alignment were manually removed, and only the ENTH and GAT domains were used to generate the phylogenetic tree. A maximum-likelihood tree of the $N$. benthamiana and $A$. thaliana TOLs was generated in MEGAX using the JTT model and with bootstrap values based on 1,000 iterations (S12 Fig). The resulting tree was then visualised using iTOL [91]. The alignment used to make the tree is provided as a S13 Data.

\section{Hairpin RNA-mediated gene silencing}

The silencing fragment was amplified out of $N$. benthamiana cDNA by Phusion High-Fidelity DNA Polymerase (Thermo Fisher Scientific) using primers listed in S10 Table. The specificity of the silencing fragment was analysed using the $N$. benthamiana genome sequence and associated gene silencing target prediction tool (SGN VIGS tool: https://vigs.solgenomics.net). The purified amplicon was cloned into pRNAi-GG vector according to Yan and colleagues [92]. Construct was verified by DNA sequencing and then transformed into A. tumefaciens strain GV3101::pM90. Silencing evading NbTOL9a $\left(\right.$ NbTOL9a ${ }^{\text {syn }}$ ) was synthesised by GENEWIZ (South Plainfield, NJ, USA) in pUC57-kan and generated according to Wu and colleagues [24]. Leaves were coinfiltrated with either pRNAi-GG::NbTOL9a or pRNAi-GG::GUS, at a final $\mathrm{OD}_{600}$ of 0.5 , together with different proteins indicated in the text with final $\mathrm{OD}_{600}$ indicated in S10 Table. The HR cell death on the leaves was scored at 5 to 7 days as described above.

\section{Protein-protein interaction studies}

Yeast two-hybrid screens. Unbiased $\mathrm{Y} 2 \mathrm{H}$ screens were performed by Hybrigenics Services (http://www.hybrigenics.com, Paris, France). For AVRcap1b (residues Ala62 -Pro678, lacking the signal peptide) was cloned into both the pB27 and pB66 bait plasmids, as C-terminal fusions to LexA (LexA-AVRcap1b) and Gal4 (Gal4-AVRcap1b), respectively. The 2 screens were performed against a randomly primed $N$. benthamiana-mixed tissue cDNA library. For LexA-AVRcap $1 b$, a total of 52.8 million interactions were screened (approximately 5 -fold library coverage), and 9 positive clones were fully analysed. For Gal4-AVRcap1b, a total of 87.6 
million interactions were screened (approximately 8 -fold library coverage), and 26 positive clones were fully analysed. These clones correspond to 14 different annotated proteins, one of which was unknown (S2 Table). For SS15 (residues Ser25 -stop 246, lacking the signal peptide) was cloned into pB27 as a C-terminal LexA bait (LexA-SS15) and screened against a randomly primed $N$. benthamiana-mixed tissue cDNA library. A total of 61.4 million interactions were screened (approximately 6-fold library coverage), and 202 positive clones were fully processed, corresponding to 10 different annotated proteins, 3 of which were unknown (S3 Table). Interactions were categorised based on their Predicted Biological Score, a measure to assess the interaction reliability, which is calculated based on a statistical model of the competition for bait binding between fragments [93,94].

To narrow down the region mediating protein-protein interactions between SS15 and NRCs, we generated NRC2, NRC3, and NRC4 CC and NB-ARC domain truncations and ran a Y2H experiment using the Matchmaker Gold system (Takara Bio, USA). Plasmid DNA encoding AVRcap1b (negative control) and SS15 in pGBKT7 (bait), generated in this study (using primers shown in S11 Table), were cotransformed into chemically competent Y2HGold cells (Takara Bio, USA) with individual NRC truncates in pGADT7 (prey) (using primers shown in S11 Table), as described previously $[95,96]$, in the AH109 yeast strain. Single colonies grown on selection plates were inoculated in $5 \mathrm{ml}$ of $\mathrm{SD}^{\text {-Leu-Trp }}$ overnight at $28^{\circ} \mathrm{C}$ (ST0047, Takara Bio, USA). Saturated culture was then used to make serial dilutions of $\mathrm{OD}_{600} 1,10^{-1}$ and $10^{-2}$, respectively. A volume of $3 \mu \mathrm{l}$ of each dilution was then spotted on a $\mathrm{SD}^{\text {-Leu-Trp }}$ plate (ST0048, Takara Bio, USA) as a growth control and on a SD ${ }^{\text {-Leu-Trp-Ade-His }}$ plate (ST0054, Takara Bio, USA) containing X- $\alpha$-gal and supplemented with $0.2 \%$ Adenine. Plates were incubated for 3 to 6 days at $28^{\circ} \mathrm{C}$ and then imaged. Each experiment was repeated a minimum of 3 times, with similar results. The commercial yeast constructs were used as positive (pGBKT753/pGADT7-T) and negative (pGBKT7-Lam/pGADT7-T) controls (Clontech, Takara Bio, CA, USA).

In planta CoIPs. Protein samples were extracted from 2 N. benthamiana leaves 3 days post-agroinfiltration and homogenised in GTEN extraction buffer [10\% glycerol, $25 \mathrm{mM}$ Tris$\mathrm{HCl}$ (pH 7.5), $1 \mathrm{mM}$ EDTA, $150 \mathrm{mM} \mathrm{NaCl}$, 2\% (w/v) polyvinylpolypyrrolidone, $10 \mathrm{mM}$ dithiothreitol, $1 \times$ protease inhibitor cocktail (SIGMA), 0.2\% IGEPAL (SIGMA, United Kingdom)] [97]. The final concentration $\mathrm{OD}_{600}$ used for each protein is indicated in $\mathrm{S} 13$ Table. After centrifugation at 5,000 $\times g$ for 20 minutes, the supernatant was passed through a Minisart $0.45 \mu \mathrm{M}$ filter (Sartorius Stedim Biotech, Goettingen, Germany) and used for SDS-PAGE. For coIP, $1.4 \mathrm{ml}$ of filtered total protein extract was mixed with $30 \mu \mathrm{l}$ of GFP-Trap-A agarose beads (Chromatek, Munich, Germany), anti-c-myc agarose beads (A7470, SIGMA) or anti-HA affinity matrix beads (Roche, Switzerland) and incubated end over end for 1 hour at $4^{\circ} \mathrm{C}$. Beads were washed 5 times with immunoprecipitation wash buffer [GTEN extraction buffer with $0.3 \%(\mathrm{v} / \mathrm{v})$ IGEPAL (SIGMA)] and resuspended in $70 \mu \mathrm{l}$ SDS loading dye. Proteins were eluted from beads by heating at 10 minutes at $70^{\circ} \mathrm{C}$ (for GFP) or $95^{\circ} \mathrm{C}$ (for myc or $\mathrm{HA}$ ). Immunoprecipitated samples were separated by SDS-PAGE and transferred onto a polyvinylidene difluoride membrane using Trans-Blot turbo Transfer system (Bio-Rad, Munich), according to the manufacturer's instructions. Blots were preblocked with $5 \%$ skim milk powder in Tris-buffered saline plus Tween 20 (TBS-T) overnight in $4^{\circ} \mathrm{C}$ or for a minimum of 1 hour at room temperature. Epitope tags were detected with HA-probe (F-7) horse radish peroxidase (HRP)conjugated antibody (Santa Cruz Biotechnology), c-Myc (9E10) HRP (Santa Cruz Biotechnology), or anti-GFP (B-2) HRP (Santa Cruz Biotechnology) antibody in a 1:5,000 dilution in 5\% skim milk powder in TBS-T. Proteins were visualised with Pierce ECL Western (32106, Thermo Fisher Scientific) and where necessary up to 50\% SuperSignal West Femto Maximum Sensitivity Substrate (34095, Thermo Fisher Scientific). Membrane imaging was carried out 
with an ImageQuant LAS 4000 luminescent imager (GE Healthcare Life Sciences, Piscataway, NJ). Rubisco loading control was stained using Pierce (24580, Thermo Fisher Scientific) or Instant Blue (Expedeon, Cambridge, United Kingdom).

Protein purification from $E$. coli and in vitro protein-protein interaction studies. Recombinant SS15 protein (lacking signal peptide) was produced using E. coli SHuffle cells [98] transformed with pOPINS3C:SS15 (see Gene cloning and synthesis section above). Cell culture was grown in autoinduction media [99] at $30^{\circ} \mathrm{C}$ to an $\mathrm{A}_{600} 0.6$ to 0.8 followed by overnight incubation at $18^{\circ} \mathrm{C}$ and harvested by centrifugation. Pelleted cells were resuspended in $50 \mathrm{mM}$ Tris $\mathrm{HCl}$ (pH 8), $500 \mathrm{mM} \mathrm{NaCl}, 50 \mathrm{mM}$ Glycine, 5\% (vol/vol) glycerol, and $20 \mathrm{mM}$ imidazole (buffer A) supplemented with cOmplete EDTA-free protease inhibitor tablets (Roche) and lysed by sonication. The clarified cell lysate was applied to a $\mathrm{Ni}^{2+}$-NTA column connected to an AKTA pure system. 6xHis+SUMO-SS15 was step eluted with elution buffer (buffer A containing $500 \mathrm{mM}$ imidazole) and directly injected onto a Superdex 200 26/600 gel filtration column preequilibrated in buffer B (20 mM HEPES ( $\mathrm{pH} 7.5), 150 \mathrm{mM} \mathrm{NaCl}$, and 1 $\mathrm{mM}$ TCEP). The fractions containing 6xHis+SUMO-SS15 were pooled and concentrated to 2 to $3 \mathrm{mg} / \mathrm{ml}$. The $6 \mathrm{xHis}+\mathrm{SUMO}$ tag was cleaved by addition of $3 \mathrm{C}$ protease $(10 \mu \mathrm{g} / \mathrm{mg}$ fusion protein) and incubation overnight at $4^{\circ} \mathrm{C}$. Cleaved $\mathrm{SS} 15$ was further purified using a $\mathrm{Ni}^{2}$ ${ }^{+}$-NTA column (collecting the eluate) followed by gel filtration as above. The concentration of protein was judged by absorbance at $280 \mathrm{~nm}$ (using a calculated molar extinction coefficient of $\left.\mathrm{SS} 15,35920 \mathrm{M}^{-1} \mathrm{~cm}^{-1}\right)$.

Recombinant NRC3 $3^{\mathrm{NB}-\mathrm{ARC}}$ was produced using E. coli Lemo21 (DE3) cells transformed with pOPINS3C:NRC $3^{\mathrm{NB}-\mathrm{ARC}}$ (see Gene cloning and synthesis section for details). Cell culture was grown in autoinduction media at $30^{\circ} \mathrm{C}$ to an $\mathrm{A}_{600} 0.6$ to 0.8 followed by overnight incubation at $18^{\circ} \mathrm{C}$ and harvested by centrifugation. Pelleted cells were resuspended in buffer A supplemented with EDTA-free protease inhibitor tablets and lysed by sonication. The clarified cell lysate was applied to a $\mathrm{Ni}^{2+}$-NTA column connected to an AKTA pure system. 6xHis+SUMO-NRC ${ }^{\text {NB-ARC }}$ was step eluted with elution buffer (buffer A containing $500 \mathrm{mM}$ imidazole) and directly injected onto a Superdex $20026 / 600$ gel filtration column preequilibrated in buffer B (20 mM HEPES (pH 7.5), $150 \mathrm{mM} \mathrm{NaCl}$, and $1 \mathrm{mM} \mathrm{TCEP).} \mathrm{The} \mathrm{fractions} \mathrm{containing}$ 6xHis+SUMO- NRC $3^{\mathrm{NB}-\mathrm{ARC}}$ were pooled and concentrated to $1 \mathrm{mg} / \mathrm{ml}$. The $6 \mathrm{xHis}+\mathrm{SUMO}$ tag was cleaved by addition of $3 \mathrm{C}$ protease $(10 \mu \mathrm{g} / \mathrm{mg}$ fusion protein) and incubation overnight at $4{ }^{\circ} \mathrm{C}$. Cleaved $\mathrm{NRC} 3^{\mathrm{NB}-\mathrm{ARC}}$ was further purified using a $\mathrm{Ni}^{2+}$-NTA column (collecting the eluate) followed by gel filtration as above. The concentration of protein was judged by absorbance at $280 \mathrm{~nm}$ (using a calculated molar extinction coefficient of NRC3 ${ }^{\mathrm{NB}-\mathrm{ARC}}, 48020$ $\mathrm{M}^{-1} \mathrm{~cm}^{-1}$ ).

To purify SS15 in complex with NRC $3^{\text {NB-ARC }}, E$. coli cultures were grown individually for SS15 and NRC ${ }^{\text {NB-ARC }}$ and harvested by centrifugation as explained above. Pelleted cells expressing SS15 and NRC $3^{\mathrm{NB}-\mathrm{ARC}}$ were resuspended separately in buffer A supplemented with EDTA-free protease inhibitor tablets. After resuspension, $\mathrm{SS} 15$ and NRC ${ }^{\mathrm{NB}-\mathrm{ARC}}$ expressing cells were mixed and lysed together by sonication. The clarified cell lysate (containing 6xHis+SUMO-NRC $3^{\text {NB-ARC }}$ and 6xHis-SUMO-SS15) was applied to a $\mathrm{Ni}^{2+}$-NTA column connected to an AKTA pure system. $6 \mathrm{xHis}+\mathrm{SUMO}$-tagged protein (SS15 and NRC3 ${ }^{\mathrm{NB}-\mathrm{ARC}}$ ) was step eluted with elution buffer (buffer A containing $500 \mathrm{mM}$ imidazole) and directly injected onto a Superdex 200 26/600 gel filtration column preequilibrated in buffer B (20 mM HEPES (pH 7.5), $150 \mathrm{mM} \mathrm{NaCl}$, and $1 \mathrm{mM}$ TCEP). The fractions containing 6xHis+SUMO-SS15 and 6xHis+SUMO-NRC $3^{\mathrm{NB}-\mathrm{ARC}}$ were pooled and concentrated to 1 to $2 \mathrm{mg} / \mathrm{ml}$. The $6 \mathrm{xHis}$ + SUMO tag was cleaved by addition of $3 \mathrm{C}$ protease $(10 \mu \mathrm{g} / \mathrm{mg}$ fusion protein) and incubation overnight at $4^{\circ} \mathrm{C}$. Cleaved $\mathrm{NRC} 3^{\mathrm{NB}-\mathrm{ARC}}$ and SS 15 complex was further purified using a $\mathrm{Ni}^{2}$ ${ }^{+}$-NTA column (collecting the eluate) followed by gel filtration as above. The fractions 
containing complex of $\mathrm{NRC} 3^{\mathrm{NB}-\mathrm{ARC}}$ and SS15 were pooled together and concentrated. The concentration of complex was judged by absorbance at $280 \mathrm{~nm}$ (using a calculated molar extinction coefficient of SS15 and NRC $3^{\mathrm{NB}-\mathrm{ARC}}, 83940 \mathrm{M}^{-1} \mathrm{~cm}^{-1}$ ).

\section{IP-MS}

Total proteins were extracted from $N$. benthamiana leaves 3 days after agroinfiltration of GFP:: AVRcap1b or GFP::PexRD54 [74] and subjected to immunoprecipitation using GFP_Trap_A beads (Chromotek, Munich, Germany), as described previously [50,97,100]. PexRD54 was included as a control, as it is also a large $P$. infestans RxLR effector and extensive studies suggests that its role is likely independent of the NRC network [74,75]. Final $\mathrm{OD}_{600}$ for each protein is indicated in $\mathrm{S} 13$ Table. Immunoprecipitated samples were separated by SDS-PAGE (4\% to $20 \%$ gradient gel, Biorad, United Kingdom) and stained with Coomassie brilliant Blue G250 (SimplyBlue Safe stain, Invitrogen). Enriched protein samples were cut out of the gel (approximately $5 \times 10 \mathrm{~mm}$ ) and digested with trypsin. Extracted peptides were analysed by liquid chromatography-tandem mass spectrometry (LC-MS/MS) with the Orbitrap Fusion mass spectrometer and nanoflow-HPLC system U3000 (Thermo Fisher Scientific, UK) [48,50]. A total of 3 biological replicates for each sample type were submitted.

Mass spectrometry data processing. As previously described [48-50,97,100], peak lists were extracted from raw data using MS Convert [101] and peptides identified on Mascot server 2.4.1 using Mascot Daemon (Matrix Science). The peak lists were searched against the N. benthamiana genome database called Nicotiana_Benthamiana_Nbv6trPAplusSGNUniq_20170808 (398,682 sequences; 137,880,484 residues), supplemented with common contaminants. The search settings allowed tryptic peptides with up to 2 possible miscleavages and charge states $+2,+3,+4$, carbamidomethylated Cysteine (static) and oxidised Methionine (variable) modifications and monoisotopic precursor and fragment ion mass tolerances $10 \mathrm{ppm}$ and $0.6 \mathrm{Da}$, respectively. A decoy database was used to validate peptide sequence matches. The Mascot results were combined in Scaffold 4.4.0 (Proteome Software), where thresholds for peptide sequence match and inferred protein were set to exceeded $95.0 \%$ and $99 \%$, respectively, with at least 2 unique peptides identified for each protein. The aforementioned protein list with spectral counts as quantitative values was exported and further analysed in Excel (Microsoft). More information on peptides identified is available in S14 Table.

The MS proteomics data have been deposited to the ProteomeXchange Consortium via the PRIDE [102] partner repository with the dataset identifier PXD023178 and 10.6019/PXD023178.

\section{Supporting information}

S1 Fig. Effectors can suppress ETI/NTI. (A) ETI/NTI suppressing effectors counteract the activation of NLR immunity elicited by other effectors with an AVR activity (AVR effectors). The majority of effectors studied to date are known to suppress PTI by acting on various host targets involved in PTI signalling. However, we know little about the mechanisms of NTI suppressing effectors and how these effectors counteract NLR receptor functions and/or resistosome formation (B) The NRC network of Solanaceae. Helper NLRs (NRC2, NRC3, and NRC4) function redundantly with a series of $\mathrm{R}$ genes that confer resistance against multiple pathogens. These R genes encode NLR sensors that have specialised in detecting effectors from pathogens as diverse as oomycetes, bacteria, nematodes, viruses, and aphids. Many of the NRC-dependent R genes are agronomically important. Sensor NLRs (blue) with N-terminal extensions possess an additional domain at the $\mathrm{N}$-terminus, represented in dark blue. The extent to which pathogen effectors have evolved to target the NRC helpers are currently unknown. Figure is adapted from [24,55]. AVR, avirulence; ETI/NTI, effector/NLR-triggered 
immunity; NLR, nucleotide-binding domain and leucine-rich repeat; NRC, NLR required for cell death; PAMP, pathogen-associated molecular pattern; PRR, pattern recognition receptor; PTI, PRR-triggered immunity.

S2 Fig. Statistical analysis of cell death suppression assay for 5 candidate effectors coinfiltrated with Pto/AvrPto or Rpi-blb2/AVRblb2. Statistical analysis was conducted using besthr R library (MacLean, 2019) (S4 Data). (A-D) Each panel corresponds to results from Pto/ AvrPto or Rpi-blb2/AVRblb2 (labelled above), coexpressed with either EV (dark blue), AVRcap1b (green), HopAB2 (dark purple), PITG-15278 (light purple), SS10 (light blue), SS15 (dark orange), or SS34 (light orange), where EV was used as a negative control, labelled below plots. The left panel represents the ranked data (dots) and their corresponding means (dashed lines), with the size of each dot proportional to the number of observations for each specific value (count key below each panel). We performed bootstrap resampling tests using a lower significance cutoff of 0.025 and an upper cutoff of 0.975 . Mean ranks of test samples falling outside of these cutoffs in the control samples bootstrap population were considered significant. The panels on the right shows the distribution of 1,000 bootstrap sample rank means, where the blue areas under the curve illustrate the 0.025 and 0.975 percentiles of the distribution. EV, empty vector.

(TIF)

S3 Fig. HopAB2 suppression of Prf-mediated cell death in N. benthamiana is not evident in young leaves. P. syringae effectors HopA1, HopAB1, HopAB2, HopAB3, and HopAF1 were transiently coexpressed with Pto/AvrPto in N. benthamiana leaves. The leaves were photographed 5 days after infiltration. HopAB2 suppression of Pto/AvrPto in N. benthamiana were evident on leaf 5 (old leaf, left), but not on leaf 3 (young leaf, right) as defined by [39]. Photographs are representative of 3 technical repeats. EV, empty vector.

S4 Fig. AVRcap1b and SS15 suppress cell death activity mediated by autoactive $\mathrm{NRC} 2^{\mathrm{H} 480 \mathrm{R}}$ and NRC $3^{\mathrm{D} 480 \mathrm{~V}}$, but not of $\mathrm{NRC}^{\mathrm{D478V}}$ mutants. (A-F) Leaf panels: Photo of representative $N$. benthamiana leaves showing $\mathrm{HR}$ after coexpression of autoimmune $\mathrm{NRC} 2{ }^{\mathrm{H} 480 \mathrm{R}}, \mathrm{NRC} 3^{\mathrm{D} 480 \mathrm{~V}}$, and $\mathrm{NRC} 4^{\mathrm{D} 478 \mathrm{~V}}$ mutants, indicated to the left of leaf panels, with either EV, AVRcap1b, PITG_15278, HopAB2, SS10, SS15, or SS34 (labelled on leaf) (S4 Data). Middle panels: HR was scored 5 days post-agroinfiltration, using a modified 0-7 scale (Segretin and colleagues) and photographed at 5 days after agroinfiltration. HR results are presented as dot plots, where the size of each dot is proportional to the number of samples with the same score (count) within each biological replicate. Each effector is represented by a different dot colour (see key right side of plot). The experiment was independently repeated 3 times each with 6 technical replicates. The columns of each tested effector correspond to results from different biological replicates. Right panels: Statistical analysis was conducted using besthr $\mathrm{R}$ library (MacLean, 2019). The dots represent the ranked data and their corresponding means (dashed lines), with the size of each dot proportional to the number of observations for each specific value (count key below each panel). The panels on the right show the distribution of 1,000 bootstrap sample rank means, where the blue areas under the curve illustrates the 0.025 and 0.975 percentiles of the distribution. A difference is considered significant if the ranked mean for a given condition falls within or beyond the blue percentile of the mean distribution of the EV control. Significant differences between the conditions are indicated with an asterisk $\left.{ }^{*}\right)$. EV, empty vector; HR, hypersensitive response.

(TIF) 
S5 Fig. Statistical analysis of cell death suppression assay for AVRcap1b and SS15 coexpressed with autoactive $\mathrm{NRC2}{ }^{\mathrm{H} 480 \mathrm{R}}, \mathrm{NRC}^{\mathrm{D} 480 \mathrm{~V}}$, and $\mathrm{NRC} 4^{\mathrm{D} 478 \mathrm{~V}}$. Statistical analysis was conducted using besthr R library (MacLean, 2019) (S5 Data). Each panel corresponds to results from (A, D) NRC2 ${ }^{\mathrm{H} 480 \mathrm{R}},(\mathrm{B}, \mathrm{E}) \mathrm{NRC} 3^{\mathrm{D} 480 \mathrm{~V}}$, or (C, F) NRC4 ${ }^{\mathrm{D} 478 \mathrm{~V}}$ (labelled above), coexpressed with either EV (blue), AVRcap1b (green), or SS15 (orange), where EV was used as a negative control (labelled under plots). The left panel represents the ranked data (dots) and their corresponding means (dashed lines), with the size of each dot proportional to the number of observations for each specific value (count key below each panel). The panels on the right show the distribution of 1,000 bootstrap sample rank means, where the blue areas under the curve illustrate the 0.025 and 0.975 percentiles of the distribution. A difference is considered significant if the ranked mean for a given condition falls within or beyond the blue percentile of the mean distribution of the EV control. EV, empty vector.

S6 Fig. Statistical analysis of suppression of NRC2/3-dependent sensor NLRs by AVRcap1b or SS15. Statistical analysis was conducted using besthr R library (MacLean, 2019) (S6 Data). (A-D) Within each panel results from a range of different sensor NLRs (labelled below) were coexpressed with either EV (blue), AVRcap1b (green), or SS15 (orange), where EV was used as a negative control (see key on right hand side). Panels (A) and (C) represent the ranked data (dots) and their corresponding means (dashed lines), with the size of each dot proportional to the number of observations for each specific value (refer to count key, on left hand side). Panels (B) and (D) show the distribution of 1,000 bootstrap sample rank means, where the blue areas under the curve illustrate the 0.025 and 0.975 percentiles of the distribution. A difference is considered significant if the ranked mean for a given condition falls within or beyond the blue percentile of the mean distribution of EV control for each treatment. EV, empty vector; NLR, nucleotide-binding domain and leucine-rich repeat.

S7 Fig. Statistical analysis shows that AVRcap1b and SS15 suppress Rx-mediated cell death in NRC4-silenced Rx-transgenic N. benthamiana plants. Statistical analysis was conducted using besthr R library (MacLean, 2019) (S7 Data). (A-F) Each panel represents a different silencing treatment (labelled above). Left plot represents the ranked data (dots) and their corresponding means (dashed lines), with the size of each dot proportional to the number of observations for each specific value (refer to count key below each individual panel). Right plot shows the distribution of 1,000 bootstrap sample rank means, where the blue areas under the curve illustrate the 0.025 and 0.975 percentiles of the distribution. A difference is considered significant if the ranked mean for a given condition falls within or beyond the blue percentile of the mean distribution of $\mathrm{EV}$ control. EV, empty vector.

S8 Fig. SS15 binds the NB-ARC domain of NRC proteins in yeast. Y2H assay of SS15 and AVRcap1b with CC and NB-ARC domains of NRC2, NRC3, and NRC4. Control plates for yeast growth are on the left (SD-Leu-Trp, ST0048, Takara Bio, USA), with quadruple dropout media supplemented with X-a-gal on the right (SD-Leu-Trp-Ade-His, ST0054, Takara Bio, USA). The commercial yeast constructs were used as positive (pGBKT7-53/pGADT7-T) and negative (pGBKT7-Lam/pGADT7-T) controls. Growth and development of blue colouration in the selection plate are both indicative of protein-protein interaction. SS15 and AVRcap1b were fused to the GAL4 DNA-binding domain (Bait), and the truncated NRCs were fused to the GAL4-activator domain (Prey). Each experiment was repeated 3 times with similar results. CC, coiled-coil; NB-ARC, nucleotide-binding domain shared with APAF-1, various R- 
proteins, and CED-4; NRC, NLR required for cell death; $\mathrm{Y} 2 \mathrm{H}$, yeast two-hybrid. (TIF)

S9 Fig. CoIP experiment between SS15 and NRC2, NRC3, and NRC4. N-terminally 4xHAtagged SS15 (SS15) and SS9 (SS9) were individually transiently coexpressed with NRC proteins fused to a C-terminal 4xMyc tag. SS9 was used as a negative control. IP were performed with agarose beads conjugated to either anti-Myc (MYC IP) or anti-HA (HA IP) antibodies. Total protein extracts were immunoblotted with appropriate antisera labelled on the left. Approximate molecular weights $(\mathrm{kDa})$ of the proteins are shown on the right. Rubisco loading controls were conducted using $\mathrm{CBB}$ staining. This experiment is a representative of 2 independent replicates. CBB, Coomassie brilliant blue; coIP, co-immunoprecipitation; IP, immunoprecipitation.

(TIF)

S10 Fig. SS15 associates with NRC2, NRC3, and NRC4 p-loop mutants in planta. (A) N-terminally 4xHA-tagged SS15 was transiently coexpressed in $\mathrm{N}$. benthamiana with C-terminally 4xMyc-tagged NRC2, NRC4, NRC2 ${ }^{\mathrm{K} 188 \mathrm{R}}, \mathrm{NRC}^{\mathrm{K} 191 \mathrm{R}}$, and NRC4 ${ }^{\mathrm{K} 190 \mathrm{R}}$. IP was performed with agarose beads conjugated to anti-Myc (Myc IP) antibodies. Total protein extracts (Input) and proteins obtained by coIP were immunoblotted with appropriate antisera labelled on the right. Approximate molecular weights $(\mathrm{kDa})$ of the proteins are shown on the right. Rubisco loading controls were conducted using CBB staining. This experiment is representative of 3 independent replicates. CBB, Coomassie brilliant blue; coIP, co-immunoprecipitation; IP, immunoprecipitation.

S11 Fig. Target of AVRcap1b identified via IP-MS. (A) Immune affinity enriched protein abundance plot generated based on proteins groups identified for AVRcaplb and PexRD54. Protein abundances were calculated as the total mean spectral counts identified with each effector across 3 replicates. The $\log _{2}$ of the ratio of mean counts observed for AVRcaplb divided by the mean counts observed for PexRD54 was calculated. All 261 proteins identified were then sorted from highest to lowest ratio and plotted in this order (see S14 Table for details). Proteins with the highest ratio correspond to the most likely AVRcaplb (blue) specific interactors. Proteins most likely to correspond to PexRD54 are labelled in green. Proteins in grey were identified in both effectors. (B) Eight putative unique AVRcaplb proteins (based on previously mentioned ratio of mean spectral counts) identified in IP-MS experiment. Unique spectral counts obtained in each of the 3 independent replicates with AVRcaplb are included on the right-hand side. IP-MS, immunoprecipitation-mass spetrometry. (TIF)

S12 Fig. Maximum-likelihood phylogenetic tree of TOL proteins from $N$. benthamiana and A. thaliana. Protein sequences were aligned using Clustal Omega. The ENTH and GAT domains were used for further analysis. The phylogenetic tree was constructed in MEGAX using the JTT substitution model and 1,000 bootstrap iterations. Branches with bootstrap support higher than 80 are indicated with red dots. NbTOL9a is indicated by 2 red asterisks. The scale bar indicates the evolutionary distance in amino acid substitutions per site. JTT, JonesTaylor-Thornton; TOL, Target of Myb 1-like protein.

S13 Fig. RNAi::NbTOL9a silencing construct effectively reduces protein accumulation levels of NbTOL9a. WT NbTOL9a (NbTOL9a::6HA) and the synthetic version (synNbTOL9a::6xHA) were transiently coexpressed with RNAi::GUS or RNAi:NbTOL9a. 
synNbTOL9a::6xHA was used as a control that is not targeted for knockdown by the RNAi:: NbTOL9a. Total protein extracts were immunoblotted with HA antiserum. Approximate molecular weights $(\mathrm{kDa})$ of the proteins are shown on the right. Rubisco loading controls were conducted using Pierce staining. WT, wild type.

(TIF)

S14 Fig. Statistical analysis showing silencing of NbTOL9a enhanced cell death mediated by NRC2 ${ }^{\mathrm{H} 480 \mathrm{R}}$ and $\mathrm{NRC} 3^{\mathrm{D480V}}$ but not ZAR1 ${ }^{\mathrm{D} 481 \mathrm{~V}}$ or $\mathrm{NRC4}^{\mathrm{D} 478 \mathrm{~V}}$. Statistical analysis was conducted using besthr R library (MacLean, 2019) (S10 Data). Each panel represents increasing concertation of A. tumefaciens expressing NRC $3^{\mathrm{D} 480 \mathrm{~V}}$ and NRC4 ${ }^{\mathrm{D} 478 \mathrm{~V}}\left(\mathrm{OD}_{600}=0.1,0.25\right.$, or 0.5). (A) $\mathrm{NRC} 2^{\mathrm{H} 480 \mathrm{R}}$, (B) $\mathrm{NRC} 3^{\mathrm{D} 480 \mathrm{~V}}$, (C) NRC4 ${ }^{\mathrm{D} 478 \mathrm{~V}}$, and (D) ZAR1 ${ }^{\mathrm{D} 481 \mathrm{~V}}$. The left most panel represents the ranked data (dots) and their corresponding means (dashed lines), with the size of each dot proportional to the number of observations for each specific value (refer to count legend bottom of figure). The right panel shows the distribution of 1,000 bootstrap sample rank means, where the blue areas under the curve illustrate the 0.025 and 0.975 percentiles of the distribution. A difference is considered significant if the ranked mean for RNAi:: NbTOL $9 a$ (purple) falls within or beyond the blue percentile of the mean distribution of RNAi::GUS (blue) for each treatment combination.

S15 Fig. Statistical analysis of HR mediated by autoactive $\mathrm{NRC}^{\mathrm{D} 480 \mathrm{~V}}$, but not MEK2 ${ }^{\mathrm{DD}}$ or $\mathrm{NRC4}^{\mathrm{D} 478 \mathrm{~V}}$, is reduced when NbTOL9a is overexpressed. Statistical analysis was conducted using besthr R library (MacLean, 2019) (S11 Data). For (A) MEK2 ${ }^{\mathrm{DD}}$, (B) NRC3 ${ }^{\mathrm{D} 480 \mathrm{~V}}$, and (C) $\mathrm{NRC}^{\mathrm{D} 478 \mathrm{~V}}$, the left most panel represents the ranked data (dots) and their corresponding means (dashed lines), with the size of each dot proportional to the number of observations for each specific value (refer to count legend bottom of figure). The right panel shows the distribution of 1,000 bootstrap sample rank means, where the blue areas under the curve illustrate the 0.025 and 0.975 percentiles of the distribution. A difference is considered significant if the ranked mean for AVRcap1b (purple) falls within or beyond the blue percentile of the mean distribution of EV control (blue) for each treatment $\left(\mathrm{MEK}_{2}{ }^{\mathrm{DD}}, \mathrm{NRC}^{\mathrm{D} 480 \mathrm{~V}}\right.$, and $\mathrm{NRC}^{2}{ }^{\mathrm{D} 478 \mathrm{~V}}$ ), in the presence of either EV or NbTOL9a. EV, empty vector; HR, hypersensitive response. (TIF)

S16 Fig. Statistical analysis of $\mathrm{NRC}^{\mathrm{D} 480 \mathrm{~V}}$ cell death suppression mediated by AVRcap1b in NbTOL9a silencing. Statistical analysis was conducted using besthr R library (MacLean, 2019) (S12 Data). Autoactive NRC3 (NRC3 ${ }^{\mathrm{D} 480 \mathrm{~V}}$ ) was coexpressed with either EV (negative control) or AVRcaplb (labelled on bottom of plot), with RNAi::GUS (blue) or RNAi:: NbTOL9a (purple) silencing treatments. (A) represents the ranked data (dots) and their corresponding means (dashed lines), with the size of each dot proportional to the number of observations for each specific value (refer to count key, on right hand side). (B) shows the distribution of 1,000 bootstrap sample rank means, where the blue areas under the curve illustrate the 0.025 and 0.975 percentiles of the distribution. A difference is considered significant if the ranked mean for a given condition falls within or beyond the blue percentile of the mean distribution of the corresponding RNAi::GUS controls within each given treatment $\left(\mathrm{NRC} 3^{\mathrm{D} 480 \mathrm{~V}}+\mathrm{EV}, \mathrm{NRC} 3^{\mathrm{D} 480 \mathrm{~V}}+\mathrm{AVRcap} 1 \mathrm{~b}, \mathrm{NRC} 4^{\mathrm{D} 478 \mathrm{~V}}+\mathrm{EV}\right.$, and $\left.\mathrm{NRC} 4^{\mathrm{D} 478 \mathrm{~V}}+\mathrm{AVRcap} 1 \mathrm{~b}\right)$. $\mathrm{EV}$, empty vector.

(TIF)

S1 Table. List of effectors used in this study. (XLSX) 
S2 Table. List of interactors of AVRcap1b identified in $\mathrm{Y} 2 \mathrm{H}$ screen.

(XLSX)

S3 Table. List of interactors of SS15 identified in Y2H screen.

(XLSX)

S4 Table. List of interactors of AVRcap1b identified by IP-MS.

(XLSX)

S5 Table. Sequences of $N$. benthamiana TOLs used in this study. (XLSX)

S6 Table. List of primers used for $P$. infestans effector cloning. (XLSX)

S7 Table. Sequences of NRC2, NRC3, and NRC4 used in this study. (XLSX)

S8 Table. List of primers used for NRC2, NRC3, NRC4, and SW5b cloning. (XLSX)

S9 Table. List of NLRs and corresponding AVRs used in cell death assays. (XLSX)

S10 Table. List of primers used to generate NbTOL9a hairpin silencing construct. (XLSX)

S1 1 Table. List of primers used for cloning NRC Y2H constructs. (XLSX)

S12 Table. Primers used to generate NbTOL golden gate modules. (XLSX)

S13 Table. List of final $\mathrm{OD}_{600}$ used in CoIP experiments. (XLSX)

S14 Table. Spectrum reports for AVRcap1b IP-MS experiments. (XLSX)

S15 Table. Primers used for cloning NRCs into $E$. coli heterologous expression vectors. (XLSX)

S1 Data. HR score data used to generate Fig $1 \mathrm{~B}$.

(XLSX)

S2 Data. The average HR score of EV versus the average HR score of each tested effector for Pto/AvrPto, for Fig $1 \mathrm{~B}$.

(XLSX)

S3 Data. The average HR score of EV versus the average HR score of each tested effector for Rpiblb2/AVRblb2, for Fig $1 B$.

(XLSX)

S4 Data. HR score data used to generate Fig 1D, S2 and S4 Figs.

(XLSX)

S5 Data. HR score data used to generate Fig 2D, S5 Fig. (XLSX) 
S6 Data. HR score data used to generate Fig $3 B$ and 3D, S6 Fig. (XLSX)

S7 Data. HR score data used to generate Fig 4C and 4E, S7 Fig. (XLSX)

S8 Data. Data used to generate Fig $5 \mathrm{~B}$ and $5 \mathrm{C}$. (XLSX)

S9 Data. Output for statistics for Fig $5 \mathrm{~B}$ and $5 \mathrm{C}$. (XLSX)

S10 Data. HR score data used to generate Fig 10B, S10 Fig. (XLSX)

S11 Data. HR score data used to generate Fig 11B, S11 Fig. (XLSX)

S12 Data. HR score data used to generate Fig 12B, S12 Fig. (XLSX)

S13 Data. Alignment used to generate phylogenetic tree of A. thaliana and N. benthamiana TOL proteins.

(PHY)

\section{Acknowledgments}

We thank Aleksandra "Ola” Białas (The Sainsbury Laboratory, Norwich, UK) for valuable comments on figures for this paper and Adeline Harant for technical assistance.

\section{Author Contributions}

Conceptualization: Lida Derevnina, Mauricio P. Contreras, Chih-Hang Wu, Sophien Kamoun.

Formal analysis: Lida Derevnina, Jan Skłenar, Dan MacLean.

Funding acquisition: Lida Derevnina, Hiroaki Adachi, Jessica Upson, Angel Vergara Cruces, Sophien Kamoun.

Investigation: Lida Derevnina, Mauricio P. Contreras, Hiroaki Adachi, Jessica Upson, Angel Vergara Cruces, Rongrong Xie, Abbas Maqbool, Chih-Hang Wu.

Methodology: Lida Derevnina, Mauricio P. Contreras, Hiroaki Adachi, Jan Skłenar, Abbas Maqbool, Chih-Hang Wu.

Project administration: Lida Derevnina, Mauricio P. Contreras, Hiroaki Adachi, Chih-Hang Wu, Sophien Kamoun.

Resources: Lida Derevnina, Hiroaki Adachi, Jessica Upson, Angel Vergara Cruces, Jan Skłenar, Frank L. H. Menke, Sam T. Mugford, Wenbo Ma, Saskia A. Hogenhout, Aska Goverse, Abbas Maqbool, Chih-Hang Wu, Sophien Kamoun.

Supervision: Lida Derevnina, Abbas Maqbool, Chih-Hang Wu, Sophien Kamoun.

Validation: Lida Derevnina, Mauricio P. Contreras, Jessica Upson, Sam T. Mugford, Abbas Maqbool.

Visualization: Lida Derevnina, Jan Skłenar. 
Writing - original draft: Lida Derevnina, Mauricio P. Contreras, Hiroaki Adachi, Abbas Maqbool, Sophien Kamoun.

Writing - review \& editing: Lida Derevnina, Mauricio P. Contreras, Hiroaki Adachi, Abbas Maqbool, Sophien Kamoun.

\section{References}

1. Win J, Chaparro-Garcia A, Belhaj K, Saunders DG, Yoshida K, Dong S, et al. Effector biology of plantassociated organisms: concepts and perspectives. Cold Spring Harb Symp Quant Biol. 2012; 77:23547. https://doi.org/10.1101/sqb.2012.77.015933 PMID: 23223409

2. Toruno TY, Stergiopoulos I, Coaker G. Plant-pathogen effectors: cellular probes interfering with plant defenses in spatial and temporal manners. Annu Rev Phytopathol. 2016; 54:419-41. https://doi.org/ 10.1146/annurev-phyto-080615-100204 PMID: 27359369

3. Macho AP, Zipfel C. Targeting of plant pattern recognition receptor-triggered immunity by bacterial type-III secretion system effectors. Curr Opin Microbiol. 2015; 23:14-22. https://doi.org/10.1016/j.mib. 2014.10.009 PMID: 25461568

4. Zheng X, Wagener N, McLellan H, Boevink PC, Hua C, Birch PRJ, et al. Phytophthora infestans RXLR effector SFI5 requires association with calmodulin for PTI/MTI suppressing activity. New Phytol. 2018; 219:1433-46. https://doi.org/10.1111/nph.15250 PMID: 29932222

5. Cui $\mathrm{H}$, Tsuda K, Parker JE. Effector-Triggered Immunity: from pathogen perception to robust defense. Annu Rev Plant Biol. 2015; 66:487-511. https://doi.org/10.1146/annurev-arplant-050213-040012 PMID: 25494461

6. Jones JD, Vance RE, Dangl JL. Intracellular innate immune surveillance devices in plants and animals. Science. 2016;354. https://doi.org/10.1126/science.aaf6395 PMID: 27934708

7. Zhang X, Dodds PN, Bernoux M. What do we know about NOD-like receptors in plant immunity? Annu Rev Phytopathol. 2017; 55:205-29. https://doi.org/10.1146/annurev-phyto-080516-035250 PMID: 28637398

8. Cesari S. Multiple strategies for pathogen perception by plant immune receptors. New Phytol. 2018; 219:17-24. https://doi.org/10.1111/nph.14877 PMID: 29131341

9. Kourelis J, van der Hoorn RAL. Defended to the nines: 25 years of resistance gene cloning identifies nine mechanisms for R protein function. Plant Cell. 2018; 30:285. https://doi.org/10.1105/tpc.17. 00579 PMID: 29382771

10. Dodds PN, Rathjen JP. Plant immunity: towards an integrated view of plant-pathogen interactions. Nat Rev Genet. 2010; 11:539-48. https://doi.org/10.1038/nrg2812 PMID: 20585331

11. Jones JD, Dangl JL. The plant immune system. Nature. 2006; 444:323-9. https://doi.org/10.1038/ nature05286 PMID: 17108957

12. Thordal-Christensen $\mathrm{H}$. A holistic view on plant effector-triggered immunity presented as an iceberg model. Cell Mol Life Sci. 2020; 77:3963-76. https://doi.org/10.1007/s00018-020-03515-w PMID: 32277261

13. Bos JIB, Kanneganti T-D, Young C, Cakir C, Huitema E, Win J, et al. The C-terminal half of Phytophthora infestans RXLR effector AVR3a is sufficient to trigger R3a-mediated hypersensitivity and suppress INF1-induced cell death in Nicotiana benthamiana. Plant J. 2006; 48:165-76. https://doi.org/ 10.1111/j.1365-313X.2006.02866.x PMID: 16965554

14. D’Ambrosio JM, Couto D, Fabro G, Scuffi D, Lamattina L, Munnik T, et al. Phospholipase C2 affects MAMP-triggered immunity by modulating ROS production. Plant Physiol. 2017; 175:970-81. https:// doi.org/10.1104/pp.17.00173 PMID: 28827453

15. Harris JM, Balint-Kurti P, Bede JC, Day B, Gold S, Goss EM, et al. What are the top 10 unanswered questions in molecular plant-microbe interactions? Mol Plant-Microbe Interact. 2020; 33:1354-65. https://doi.org/10.1094/MPMI-08-20-0229-CR PMID: 33106084

16. Kourelis J, Kamoun S. RefPlantNLR: a comprehensive collection of experimentally validated plant NLRs. bioRxiv. 2020. https://doi.org/10.1101/2020.07.08.193961

17. Shao ZQ, Xue JY, Wu P, Zhang YM, Wu Y, Hang YY, et al. Large-scale analyses of angiosperm nucleotide-binding site-leucine-rich repeat genes reveal three anciently diverged classes with distinct evolutionary patterns. Plant Physiol. 2016; 170:2095-109. https://doi.org/10.1104/pp.15.01487 PMID: 26839128 
18. Tamborski J, Krasileva KV. Evolution of plant NLRs: from natural history to precise modifications. Annu Rev Plant Biol. 2020; 71:355-78. https://doi.org/10.1146/annurev-arplant-081519-035901 PMID: 32092278

19. Lee H-Y, Mang H, Choi E-H, Seo Y-E, Kim M-S, Oh S, et al. Genome-wide functional analysis of hot pepper immune receptors reveals an autonomous NLR cluster in seed plants. bioRxiv. 2020. https:// doi.org/10.1101/2019.12.16.878959

20. Bentham AR, Zdrzalek R, De la Concepcion JC, Banfield MJ. Uncoiling CNLs: Structure/function approaches to understanding CC domain function in plant NLRs. Plant Cell Physiol. 2018; 59:2398408. https://doi.org/10.1093/pcp/pcy185 PMID: 30192967

21. Bentham $A$, Burdett $H$, Anderson PA, Williams SJ, Kobe B. Animal NLRs provide structural insights into plant NLR function. Ann Bot. 2017; 119:827-702. https://doi.org/10.1093/aob/mcw171 PMID: 27562749

22. Sarris PF, Cevik V, Dagdas G, Jones JD, Krasileva KV. Comparative analysis of plant immune receptor architectures uncovers host proteins likely targeted by pathogens. BMC Biol. 2016; 14:8. https:// doi.org/10.1186/s12915-016-0228-7 PMID: 26891798

23. Adachi H, Derevnina L, Kamoun S. NLR singletons, pairs, and networks: evolution, assembly, and regulation of the intracellular immunoreceptor circuitry of plants. Curr Opin Plant Biol. 2019; 50:121-31. https://doi.org/10.1016/j.pbi.2019.04.007 PMID: 31154077

24. Wu CH, Abd-El-Haliem A, Bozkurt TO, Belhaj K, Terauchi R, Vossen JH, et al. NLR network mediates immunity to diverse plant pathogens. Proc Natl Acad Sci U S A. 2017; 114:8113-8. https://doi.org/10. 1073/pnas.1702041114 PMID: 28698366

25. Adachi $\mathrm{H}$, Contreras MP, Harant $\mathrm{A}, \mathrm{Wu} \mathrm{CH}$, Derevnina L, Sakai T, et al. An N-terminal motif in NLR immune receptors is functionally conserved across distantly related plant species. eLife. 2019; 8. https://doi.org/10.7554/eLife.49956 PMID: 31774397

26. Wu CH, Belhaj K, Bozkurt TO, Birk MS, Kamoun S. Helper NLR proteins NRC2a/b and NRC3 but not NRC1 are required for Pto-mediated cell death and resistance in Nicotiana benthamiana. New Phytol. 2016; 209:1344-52. https://doi.org/10.1111/nph.13764 PMID: 26592988

27. Martin R, Qi T, Zhang H, Liu F, King M, Toth C, et al. Structure of the activated ROQ1 resistosome directly recognizing the pathogen effector XopQ. Science. 2020; 370(6521):eabd9993. https://doi.org/ 10.1126/science.abd9993 PMID: 33273074

28. Wang J, Hu M, Wang J, Qi J, Han Z, Wang G, et al. Reconstitution and structure of a plant NLR resistosome conferring immunity. Science. 2019; 364:aav5870. https://doi.org/10.1126/science.aav5870 PMID: 30948527

29. Wang J, Wang J, Hu M, Wu S, Qi J, Wang G, et al. Ligand-triggered allosteric ADP release primes a plant NLR complex. Science. 2019; 364:aav5868. https://doi.org/10.1126/science.aav5868 PMID: 30948526

30. Ma S, Lapin D, Liu L, Sun Y, Song W, Zhang X, et al. Direct pathogen-induced assembly of an NLR immune receptor complex to form a holoenzyme. Science. 2020; 370:eabe3069. https://doi.org/10. 1126/science.abe3069 PMID: 33273071

31. He J, Ye W, Choi DS, Wu B, Zhai Y, Guo B, et al. Structural analysis of Phytophthora suppressor of RNA silencing 2 (PSR2) reveals a conserved modular fold contributing to virulence. Proc Natl Acad Sci U S A. 2019; 116:8054-9. https://doi.org/10.1073/pnas.1819481116 PMID: 30926664

32. Rietman H. Putting the Phytophthora infestans genome sequence at work: multiple novel avirulence and potato resistance gene candidates revealed. Wageningen University; 2011. Available from: https://library.wur.nl/WebQuery/wurpubs/406778

33. Yoshida K, Schuenemann VJ, Cano LM, Pais M, Mishra B, Sharma R, et al. The rise and fall of the Phytophthora infestans lineage that triggered the Irish potato famine. eLife. 2013; 2:e00731. https:// doi.org/10.7554/eLife.00731 PMID: 23741619

34. Haas BJ, Kamoun S, Zody MC, Jiang RH, Handsaker RE, Cano LM, et al. Genome sequence and analysis of the Irish potato famine pathogen Phytophthora infestans. Nature. 2009; 461:393-8. https:// doi.org/10.1038/nature08358 PMID: 19741609

35. Ali S, Magne M, Chen S, Obradovic N, Jamshaid L, Wang X, et al. Analysis of Globodera rostochiensis effectors reveals conserved functions of SPRYSEC proteins in suppressing and eliciting plant immune responses. Front Plant Sci. 2015; 6:623. https://doi.org/10.3389/fpls.2015.00623 PMID: 26322064

36. Postma WJ, Slootweg EJ, Rehman S, Finkers-Tomczak A, Tytgat TO, van Gelderen K, et al. The effector SPRYSEC-19 of Globodera rostochiensis suppresses CC-NB-LRR-mediated disease resistance in plants. Plant Physiol. 2012; 160:944-54. https://doi.org/10.1104/pp.112.200188 PMID: 22904163 
37. Eves-van den Akker S, Laetsch DR, Thorpe P, Lilley CJ, Danchin EG, Da Rocha M, et al. The genome of the yellow potato cyst nematode, Globodera rostochiensis, reveals insights into the basis of parasitism and virulence. Genome Biol. 2016; 17:124. https://doi.org/10.1186/s13059-016-0985-1 PMID: 27286965

38. Abramovitch RB, Kim Y-J, Chen S, Dickman MB, Martin GB. Pseudomonas type III effector AvrPtoB induces plant disease susceptibility by inhibition of host programmed cell death. EMBO J. 2003; 22:60-9. https://doi.org/10.1093/emboj/cdg006 PMID: 12505984

39. Ma L, Lukasik E, Gawehns F, Takken FLW. /the use of agroinfiltration for transient expression of plant resistance and fungal effector proteins in nicotiana benthamiana leaves. In: Bolton MD, Thomma BPHJ, editors. Plant Fungal Pathogens: Methods and Protocols. Totowa, NJ: Humana Press. 2012. p. 61-74.

40. van Ooijen G, Mayr G, Kasiem MM, Albrecht M, Cornelissen BJ, Takken FL. Structure-function analysis of the NB-ARC domain of plant disease resistance proteins. J Exp Bot. 2008; 59:1383-97. https:// doi.org/10.1093/jxb/ern045 PMID: 18390848

41. Besthr MacLean D. Zenodo. 2019. https://doi.org/10.5281/zenodo.3374507

42. Bendahmane $A$, Kanyuka K, Baulcombe DC. The Rx gene from potato controls separate virus resistance and cell death responses. Plant Cell. 1999; 11:781-91. https://doi.org/10.1105/tpc.11.5.781 PMID: 10330465

43. Tameling WIL, Baulcombe DC. Physical association of the NB-LRR resistance protein Rx with a Ran GTPase-Activating protein is required for extreme resistance to Potato virus X. Plant Cell. 2007; 19:1682-94. https://doi.org/10.1105/tpc.107.050880 PMID: 17526750

44. Tameling WIL, Nooijen C, Ludwig N, Boter M, Slootweg E, Goverse A, et al. RanGAP2 mediates nucleocytoplasmic partitioning of the NB-LRR immune receptor Rx in the solanaceae, thereby dictating Rx function. Plant Cell. 2010; 22:4176-94. https://doi.org/10.1105/tpc.110.077461 PMID: 21169509

45. Sacco MA, Mansoor $S$, Moffett $P$. A RanGAP protein physically interacts with the NB-LRR protein $R x$, and is required for Rx-mediated viral resistance. Plant J. 2007; 52:82-93. https://doi.org/10.1111/j. 1365-313X.2007.03213.x PMID: 17655649

46. Du J, Rietman $\mathrm{H}$, Vleeshouwers VGAA. Agroinfiltration and PVX agroinfection in potato and Nicotiana benthamiana. J Vis Exp. 2014; 83:e50971. https://doi.org/10.3791/50971 PMID: 24430891

47. Tameling WIL, Elzinga SDJ, Darmin PS, Vossen JH, Takken FLW, Haring MA, et al. The tomato R gene products I-2 and Ml-1 are functional ATP binding proteins with ATPase activity. Plant Cell. 2002; 14:2929-39. https://doi.org/10.1105/tpc.005793 PMID: 12417711

48. Petre B, Saunders DG, Sklenar J, Lorrain C, Win J, Duplessis S, et al. Candidate effector proteins of the rust pathogen Melampsora larici-populina target diverse plant cell compartments. Mol PlantMicrobe Interact. 2015; 28:689-700. https://doi.org/10.1094/MPMI-01-15-0003-R PMID: 25650830

49. Petre B, Contreras M, Bozkurt T, Schattat M, Sklenar J, Schornack S, et al. Host-interactor screens of Phytophthora infestans RXLR proteins reveal vesicle trafficking as a major effector-targeted process. Plant Cell. 2021. Koab069 https://doi.org/10.1093/plcell/koab069 PMID: 33677602

50. Zess EK, Jensen C, Cruz-Mireles N, De la Concepcion JC, Sklenar J, Stephani M, et al. N-terminal beta-strand underpins biochemical specialization of an ATG8 isoform. PLoS Biol. 2019; 17:e3000373. https://doi.org/10.1371/journal.pbio.3000373 PMID: 31329577

51. Isono E. TOL Keepers for ubiquitin-mediated trafficking routes in plant cells. Mol Plant. 2020; 13:6857. https://doi.org/10.1016/j.molp.2020.04.005 PMID: 32311531

52. Mosesso N, Nagel M-K, Isono E. Ubiquitin recognition in endocytic trafficking-with or without ESCRT0. J Cell Sci. 2019; 132:jcs232868. https://doi.org/10.1242/jcs.232868 PMID: 31416855

53. Moulinier-Anzola J, Schwihla M, De-Araújo L, Artner C, Jörg L, Konstantinova N, et al. TOLs function as ubiquitin receptors in the early steps of the ESCRT pathway in higher plants. Mol Plant. 2020; 13:717-31. https://doi.org/10.1016/j.molp.2020.02.012 PMID: 32087370

54. Harant A, Sakai T, Kamoun S, Adachi H. A vector system for fast-forward in vivo studies of the ZAR1 resistosome in the model plant Nicotiana benthamiana. bioRxiv. 2020. https://doi.org/10.1101/2020. 05.15.097584

55. Wu C-H, Derevnina L, Kamoun S. Receptor networks underpin plant immunity. Science. 2018; 360:1300. https://doi.org/10.1126/science.aat2623 PMID: 29930125

56. Witek K, Lin X, Karki HS, Jupe F, Witek Al, Steuernagel B, et al. A complex resistance locus in Solanum americanum recognizes a conserved Phytophthora effector. Nature Plants. 2021; 7:198-208. https://doi.org/10.1038/s41477-021-00854-9 PMID: 33574576 
57. Ding P, Sakai T, Shrestha RK, Perez NM, Guo W, Ngou BPM, et al. Chromatin accessibility landscapes activated by cell surface and intracellular immune receptors. bioRxiv. 2020. https://doi.org/10. 1101/2020.06.17.157040

58. Ngou BPM, Ahn H-K, Ding P, Jones JDG. Mutual potentiation of plant immunity by cell-surface and intracellular receptors. Nature. 2021; 592:110-5. https://doi.org/10.1038/s41586-021-03315-7 PMID: 33692545

59. Pruitt RN, Zhang L, Saile SC, Karelina D, Fröhlich K, Wan W-L, et al. Arabidopsis cell surface LRR immune receptor signaling through the EDS1-PAD4-ADR1 node. bioRxiv. 2020. https://doi.org/10. 1101/2020.11.23.391516

60. Yuan M, Jiang Z, Bi G, Nomura K, Liu M, Wang Y, et al. Pattern-recognition receptors are required for NLR-mediated plant immunity. Nature. 2021; 592:105-9. https://doi.org/10.1038/s41586-021-033166 PMID: 33692546

61. Juliana C, Fernandes-Alnemri T, Kang S, Farias A, Qin F, Alnemri ES. Non-transcriptional priming and deubiquitination regulate NLRP3 inflammasome activation. J Biol Chem. 2012; 287:36617-22. https:// doi.org/10.1074/jbc.M112.407130 PMID: 22948162

62. Yen H, Sugimoto N, Tobe T. Enteropathogenic Escherichia coli uses NleA to inhibit NLRP3 inflammasome activation. PLoS Pathog. 2015; 11:e1005121. https://doi.org/10.1371/journal.ppat.1005121 PMID: 26332984

63. Chen Y, Liu Z, Halterman DA. Molecular determinants of resistance activation and suppression by Phytophthora infestans effector IPI-O. PLoS Pathog. 2012; 8:e1002595. https://doi.org/10.1371/ journal.ppat.1002595 PMID: 22438813

64. Karki HS, Abdullah S, Chen Y, Halterman DA. Natural genetic diversity in the potato resistance gene RB confers suppression avoidance from Phytophthora effector IPI-O4. Mol Plant-Microbe Interact. 2021. https://doi.org/10.1094/MPMI-11-20-0313-R PMID: 33970667

65. Coll RC, Hill JR, Day CJ, Zamoshnikova A, Boucher D, Massey NL, et al. MCC950 directly targets the NLRP3 ATP-hydrolysis motif for inflammasome inhibition. Nat Chem Biol. 2019; 15:556-9. https://doi. org/10.1038/s41589-019-0277-7 PMID: 31086327

66. Tapia-Abellan A, Angosto-Bazarra D, Martinez-Banaclocha $\mathrm{H}$, de Torre-Minguela C, Ceron-Carrasco $\mathrm{JP}$, Perez-Sanchez H, et al. MCC950 closes the active conformation of NLRP3 to an inactive state. Nat Chem Biol. 2019; 15:560-4. https://doi.org/10.1038/s41589-019-0278-6 PMID: 31086329

67. Winter V, Hauser M-T. Exploring the ESCRTing machinery in eukaryotes. Trends Plant Sci. 2006; 11:115-23. https://doi.org/10.1016/j.tplants.2006.01.008 PMID: 16488176

68. De Craene J-O, Ripp R, Lecompte O, Thompson JD, Poch O, Friant S. Evolutionary analysis of the ENTH/ANTH/VHS protein superfamily reveals a coevolution between membrane trafficking and metabolism. BMC Genomics. 2012; 13:297. https://doi.org/10.1186/1471-2164-13-297 PMID: 22748146

69. Conlan B, Stoll T, Gorman JJ, Saur I, Rathjen JP. Development of a rapid in planta BiolD system as a probe for plasma membrane-associated immunity proteins. Front Plant Sci. 2018; 9:1882. https://doi. org/10.3389/fpls.2018.01882 PMID: 30619431

70. Rühl S, Shkarina K, Demarco B, Heilig R, Santos JC, Broz P. ESCRT-dependent membrane repair negatively regulates pyroptosis downstream of GSDMD activation. Science. 2018; 362:956-60. https://doi.org/10.1126/science.aar7607 PMID: 30467171

71. Vietri M, Radulovic M, Stenmark H. The many functions of ESCRTs. Nat Rev Mol Cell Biol. 2020; 21:25-42. https://doi.org/10.1038/s41580-019-0177-4 PMID: 31705132

72. Tsuchiya K. Inflammasome-associated cell death: Pyroptosis, apoptosis, and physiological implications. Microbiol Immunol. 2020; 64:252-69. https://doi.org/10.1111/1348-0421.12771 PMID: 31912554

73. Bozkurt TO, Belhaj K, Dagdas YF, Chaparro-Garcia A, Wu C-H, Cano LM, et al. Rerouting of plant late endocytic trafficking toward a pathogen interface. Traffic. 2015; 16:204-26. https://doi.org/10.1111/ tra.12245 PMID: 25430691

74. Dagdas YF, Belhaj K, Maqbool A, Chaparro-Garcia A, Pandey P, Petre B, et al. An effector of the Irish potato famine pathogen antagonizes a host autophagy cargo receptor. eLife. 2016;5. https://doi.org/ 10.7554/eLife.10856 PMID: 26765567

75. Pandey P, Leary AY, Tümtas Y, Savage Z, Dagvadorj B, Tan E, et al. The Irish potato famine pathogen subverts host vesicle trafficking to channel starvation-induced autophagy to the pathogen interface. bioRxiv. 2020. https://doi.org/10.1101/2020.03.20.000117

76. Verzaux E, van Arkel G, Vleeshouwers VGAA, van der Vossen EAG, Niks RE, Jacobsen E, et al. High-resolution mapping of two broad-spectrum late blight resistance genes from two wild species of 
the Solanum circaeifolium group. Potato Res. 2012; 55:109-23. https://doi.org/10.1007/s11540-0129213-x

77. Zhou J-M, Zhang Y. Plant immunity: danger perception and signaling. Cell. 2020; 181:978-89. https:// doi.org/10.1016/j.cell.2020.04.028 PMID: 32442407

78. Liu S, Lenoir CJG, Amaro TMMM, Rodriguez PA, Huitema E, Bos JIB. Virulence strategies of an insect herbivore and oomycete plant pathogen converge on host E3 SUMO ligase SIZ1 to suppress plant immunity. bioRxiv. 2020. https://doi.org/10.1101/2020.06.18.159178

79. Lu R, Malcuit I, Moffett P, Ruiz MT, Peart J, Wu A-J, et al. High throughput virus-induced gene silencing implicates heat shock protein 90 in plant disease resistance. EMBO J. 2003; 22:5690-9. https:// doi.org/10.1093/emboj/cdg546 PMID: 14592968

80. Rehman S, Postma WJ, Tytgat TO, Prins P, Qin L, Overmars H, et al. A secreted SPRY domain-containing protein (SPRYSEC) from the plant-parasitic nematode Globodera rostochiensis interacts with a CC-NB-LRR protein from a susceptible tomato. Mol Plant-Microbe Interact. 2009; 22:330-40. https://doi.org/10.1094/MPMI-22-3-0330 PMID: 19245327

81. Mei Y, Wright KM, Haegeman A, Bauters L, Diaz-Granados A, Goverse A, et al. The Globodera pallida SPRYSEC effector GpSPRY-414-2 that suppresses plant defenses targets a regulatory component of the dynamic microtubule network. Front Plant Sci. 2018; 9:1019. https://doi.org/10.3389/fpls.2018. 01019 PMID: 30050557

82. Cotton JA, Lilley CJ, Jones LM, Kikuchi T, Reid AJ, Thorpe P, et al. The genome and life-stage specific transcriptomes of Globodera pallida elucidate key aspects of plant parasitism by a cyst nematode. Genome Biol. 2014; 15:R43. https://doi.org/10.1186/gb-2014-15-3-r43 PMID: 24580726

83. Berrow NS, Alderton D, Sainsbury S, Nettleship J, Assenberg R, Rahman N, et al. A versatile ligationindependent cloning method suitable for high-throughput expression screening 84. Nucleic Acids Res. 2007; 35:e45-e. https://doi.org/10.1093/nar/gkm047 PMID: 17317681

84. Kourelis J, Kaschani F, Grosse-Holz FM, Homma F, Kaiser M, van der Hoorn RAL. A homologyguided, genome-based proteome for improved proteomics in the alloploid Nicotiana benthamiana. BMC Genomics. 2019; 20:722. https://doi.org/10.1186/s12864-019-6058-6 PMID: 31585525

85. Segretin ME, Pais M, Franceschetti M, Chaparro-Garcia A, Bos JI, Banfield MJ, et al. Single amino acid mutations in the potato immune receptor R3a expand response to Phytophthora effectors. Mol Plant-Microbe Interact. 2014; 27:624-37. https://doi.org/10.1094/MPMI-02-14-0040-R PMID: 24678835

86. Liu Y, Schiff M, Dinesh-Kumar SP. Virus-induced gene silencing in tomato. Plant J. 2002; 31:777-86. https://doi.org/10.1046/j.1365-313x.2002.01394.x PMID: 12220268

87. Petre B, Saunders DG, Sklenar J, Lorrain C, Krasileva KV, Win J, et al. Heterologous expression screens in Nicotiana benthamiana identify a candidate effector of the wheat yellow rust pathogen that associates with processing bodies. PLoS ONE. 2016; 11:e0149035. https://doi.org/10.1371/journal. pone.0149035 PMID: 26863009

88. Korbei B, Moulinier-Anzola J, De-Araujo L, Lucyshyn D, Retzer K, Khan Muhammad A, et al. Arabidopsis TOL proteins act as gatekeepers for vacuolar sorting of PIN2 plasma membrane protein. Curr Biol. 2013. 23:2500-5. https://doi.org/10.1016/j.cub.2013.10.036 PMID: 24316203

89. Madeira F, Park YM, Lee J, Buso N, Gur T, Madhusoodanan N, et al. The EMBL-EBI search and sequence analysis tools APIs in 2019. Nucleic Acids Res. 2019; 47:W636-W41. https://doi.org/10. 1093/nar/gkz268 PMID: 30976793

90. Stecher G, Tamura K, Kumar S. Molecular Evolutionary Genetics Analysis (MEGA) for macOS. Mol Biol Evol. 2020; 37:1237-9. https://doi.org/10.1093/molbev/msz312 PMID: 31904846

91. Letunic I, Bork P. Interactive tree of life (iTOL) v3: an online tool for the display and annotation of phylogenetic and other trees. Nucleic Acids Res. 2016; 44:W242-W5. https://doi.org/10.1093/nar/gkw290 PMID: 27095192

92. Yan P, Shen W, Gao X, Li X, Zhou P, Duan J. High-throughput construction of intron-containing hairpin RNA vectors for RNAi in plants. PLoS ONE. 2012; 7:e38186. https://doi.org/10.1371/journal.pone. 0038186 PMID: 22675447

93. Rain JC, Selig L, De Reuse H, Battaglia V, Reverdy C, Simon S, et al. The protein-protein interaction map of Helicobacter pylori. Nature. 2001; 409:211-5. https://doi.org/10.1038/35051615 PMID: 11196647

94. Formstecher E, Aresta S, Collura V, Hamburger A, Meil A, Trehin A, et al. Protein interaction mapping: a Drosophila case study. Genome Res. 2005; 15(3):376-84. https://doi.org/10.1101/gr.2659105 PMID: 15710747 
95. De la Concepcion JC, Franceschetti M, Maqbool A, Saitoh H, Terauchi R, Kamoun S, et al. Polymorphic residues in rice NLRs expand binding and response to effectors of the blast pathogen. Nat Plants. 2018; 4:576-85. https://doi.org/10.1038/s41477-018-0194-x PMID: 29988155

96. De la Concepcion JC, Franceschetti M, MacLean D, Terauchi R, Kamoun S, Banfield MJ. Protein engineering expands the effector recognition profile of a rice NLR immune receptor. eLife. 2019; 8. https:// doi.org/10.7554/eLife.47713 PMID: 31535976

97. Win J, Kamoun S, Jones AME. Purification of effector-target protein complexes via transient expression in Nicotiana benthamiana. Methods Mol Biol. 2011; 712:181-94. https://doi.org/10.1007/978-1 61737-998-7_15 PMID: 21359809

98. Lobstein J, Emrich CA, Jeans C, Faulkner M, Riggs P, Berkmen M. SHuffle, a novel Escherichia coli protein expression strain capable of correctly folding disulfide bonded proteins in its cytoplasm. Microb Cell Factories. 2012; 11:753. https://doi.org/10.1186/1475-2859-11-56 PMID: 22569138

99. Studier FW. Protein production by auto-induction in high-density shaking cultures. Protein Expr Purif. 2005; 41:207-34. https://doi.org/10.1016/j.pep.2005.01.016 PMID: 15915565

100. Bozkurt TO, Schornack S, Win J, Shindo T, llyas M, Oliva R, et al. Phytophthora infestans effector AVRblb2 prevents secretion of a plant immune protease at the haustorial interface. Proc Natl Acad Sci U S A. 2011; 108:20832-7. https://doi.org/10.1073/pnas.1112708109 PMID: 22143776

101. Chambers MC, Maclean B, Burke R, Amodei D, Ruderman DL, Neumann S, et al. A cross-platform toolkit for mass spectrometry and proteomics. Nat Biotechnol. 2012; 30:918-20. https://doi.org/10. 1038/nbt.2377 PMID: 23051804

102. Perez-Riverol Y, Csordas A, Bai J, Bernal-Llinares M, Hewapathirana S, Kundu DJ, et al. The PRIDE database and related tools and resources in 2019: improving support for quantification data. Nucleic Acids Res. 2019; 47:D442-D50. https://doi.org/10.1093/nar/gky1106 PMID: 30395289 